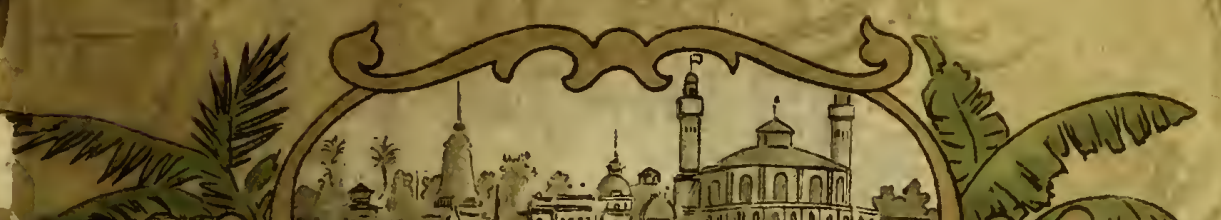

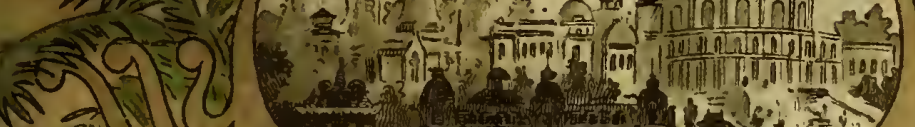

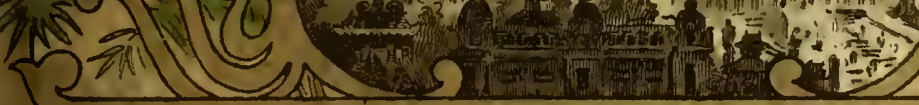

3i. An

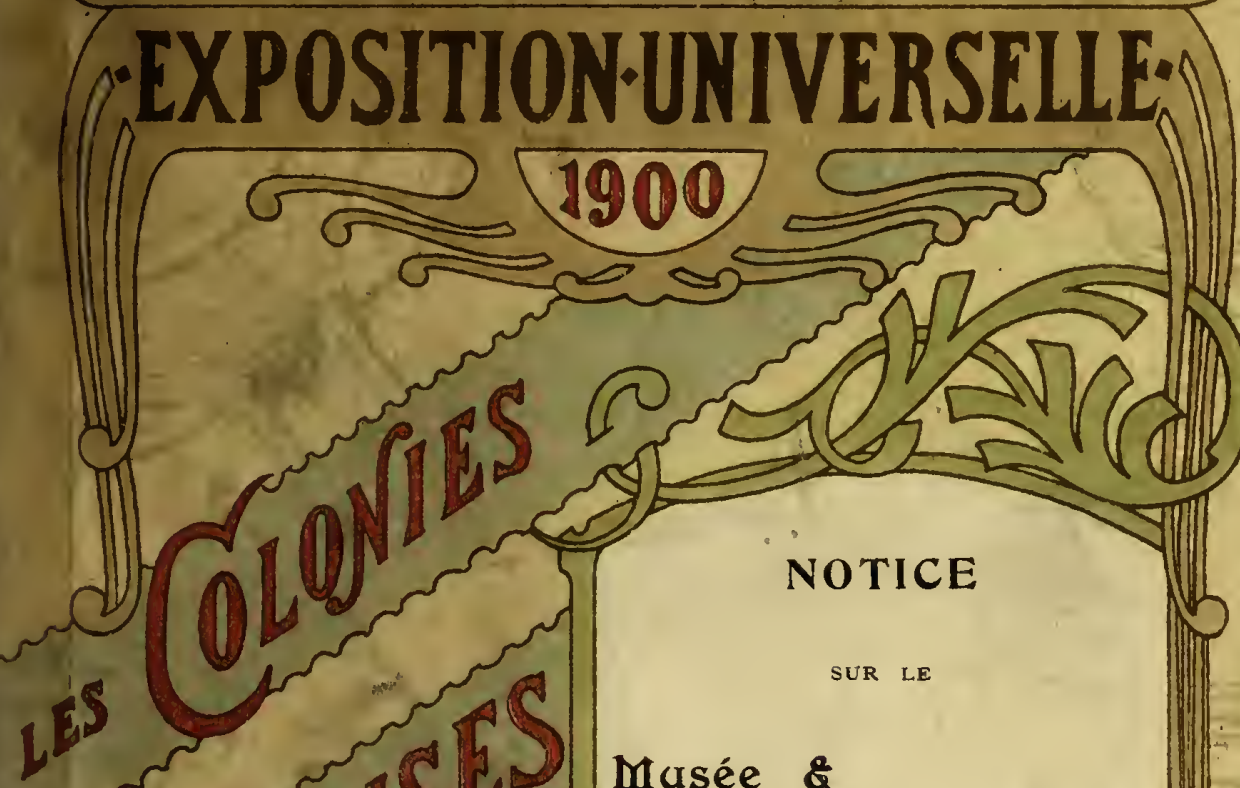

Musée \&

l'Instifut Colonial

de Marseille

Publiée à l'occasion de

l'Exposition Universplle

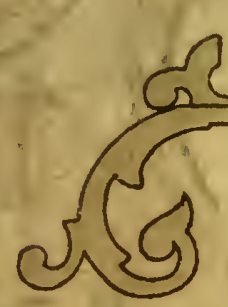

de 1900

PANL MERWART. INUTERINXI 


Digitized by the Internet Archive in 2018 with funding from Getty Research Institute 


\section{L'Institut et}

\section{le Musée Colonial}

\section{de Marseille}

\section{RO T I G par MM.}

EDOUARD HECKEL, Directeur-Fondateur, professeur à l Unwersité

JUMELLE. JACOB DE CORDEMOY, LAURENT,

Professeurs attachés à cet Institut

et M. EBERLIN, Conservateur du Musee

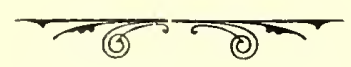

PARIS

IMPRIMERIE TYPOGRAPHIQUE HENRI ROBERGE 235, Rue du Faubourg Saint-Martin 





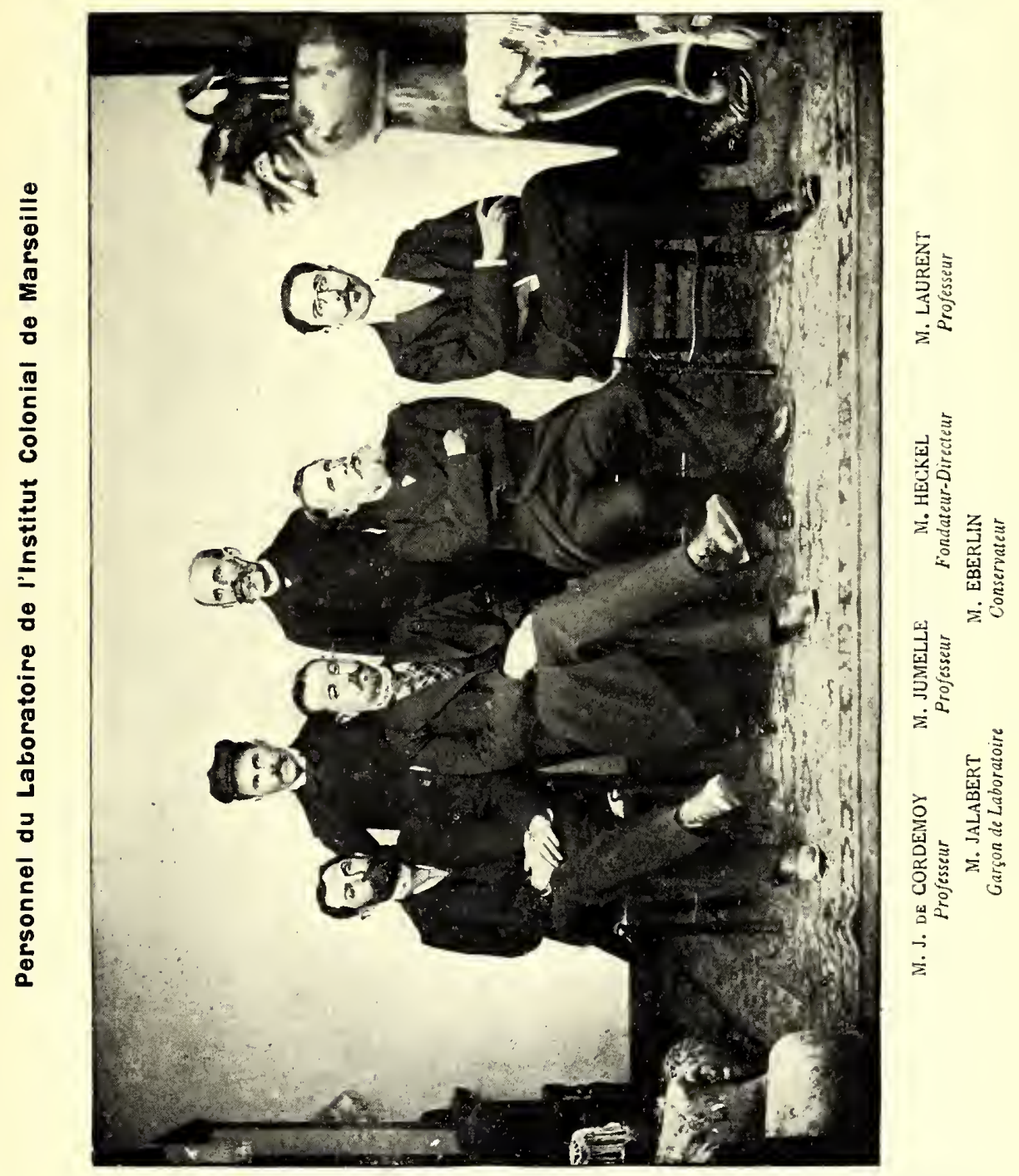

$$
\text { C. F.E. }
$$





\section{HISTORIQUE}

L'histoire du Musée et de l'Institut colonial de Marseille ne peut être que fort simple. Sa création se résume, en effet, en une œuvre personnelle et récente. Affranchi par cela même dès sa naissance des embarras et des retards qui résultent toujours du conflit des idées et des personnes, réalísé sur un plan longuement étudié et par un colonial de vieille date, il a parcouru rapidement les phases évolutives qui devaient l'amener à sa constitution actuelle; celle-ci, si satısfaisante qu'elle soit, ne parait être que l'ébauche de ce qu'elle deviendra dans l'avenir. Sept années ont été employées à cet enfantement et c'est la période d'état en 1900, que cette étude a la seule prétention de faire connaitre. Mais ce septennat (1893-1900), s'il a été laborieux n'en est pas moins resté fécond, par cela mème qu'il n'était que le couronnement d'un projet longuement mûri dont la réalisation n'attendait que le concours favorable des idées ambiantes. Entreprendre cette œuvre dès 1893 , pouvait paraitre ambitieux. A cette époque les idées coloniales n'avaient pas encore pénétré profondément dans le cœur du pays. Mais il était évident, pour tout esprit clairvoyant, que le moment n'était pas éloignéoù, la période de conquête cessant, la France s'occuperait sérieusement de mettre en valeur par la science, le vaste empire colonial qui lui avait coûté tant de sang versé et tant de lourds sacrifices pécuniaires. Et c'est ce qui est arrivé. Nos colonies, comme l'a dit excellemment Bonvallot, sont une matière première qui ne vaut que par la façon 
dont on la manipule. Voilà résumées en quelques mots la génèse et la raison d'être de ce qu'est aujourd'hui, en pleine prospérité scientifique, l'Institut et le Musée colonial de Marseille.

A quel but tendait cet établissement spécial et quel moyen a-t-il mis en œuvre pour l'atteindre? Le but vers lequel il a tendu sans défaillance, avec les forces limitées d'un seul homme d'abord, puis avec le concours bénévole de quelques travailleurs désintéressés et entraînés par la toi robuste qui anime le fondateur, est de faire le bilan de nos richesses naturelles coloniales connues ou inconnues, de les mettre en évidence dans des collections bien méthodiquement et géographiquement classées, de·les mieux étudier ou de les faire connaitre enfin dans une publication périodique propre à l'Institut colonial et à caractère absolument scientifique, qui leur permette ensuite, s’il y a lieu, de prendre grande ou petite place, selon leur valeur, dans le commerce ou dans l'industrie de la métropole. Voilà l'enseignement par les yeux. La méthode suivie est celle qui a si bien réussi à d'autres nations, notamment à l'Allemagne, dont toute l'industrie s'est faite la servante de la science et marche à grands pas derrière ce guide infaillible. Il faut que nos colonies soient mises en valeur scientifiquement. Trop longtemps, celles que nous avons possédées, notamment pendant la presque totalité du $\mathrm{xix}^{\mathrm{e}}$ siècle, sont restées livrées à l'empirisme le plus complet. Aujourd'hui, la méthode scientifique pénètre partout dans nos institutions européennes, et il faut qu'elle régente l'exploitation de nos possessions d'outre-mer de la même façon qu'elle dirige et agrandit en France le domaine économique entier. Mais ce n'est pas tout. Ces collections rassemblées à grand peine dans un Musée colonial doivent servir aussi à instruire par la parole. C'est ce qu'a bien compris la Chambre de Commerce de Marseille qui, dans un mouvement de générosité, dont, tout le fait présager, elle sera la première à tirer légitime profit, n'a pas hésité à s'imposer les sacrifices pécuniaires nécessaires pour créer, en les confiant uniquement à des Universitaires, six chaires d'enseignement colonial de divers ordres, dans lesquelles domine numériquement l'enseignement consacré à l'histoire des produits végétaux, minéraux et animaux de nos possessions 
d'outre-mer (1). Cet enseignement qui a commencé à fonctionner depuis le mois de mai 1899 par des cours de produits végétaux (ce sont sinon les plus importants du moins les plus connus dans la phase agricole que traversera longtemps encore notre domaine. colonial naissant), est actuellement dans tout son épanouissement. Il servira à l'instruction de nos populations méridionales trop peu portées jusqu'ici à connaître la valeur et les origines des produits qui forment la principale matière commerciale et industrielle de leur petite capitale: Marseille. Mais il servira surtout à faire naître et à mûrir des vocations coloniales parmi les jeunes générations auxquelles l'Ecole supérieure de Commerce Marseillaise va ouvrir ses portes, par la création d'une section coloniale qui sera inaugurée dès le mois d'octobre igoo. Or, un enseignement de cet ordre ne peut ètre fructueux qu'à la condition d'être concret; de là l'indissoluble connexion qui existe entre la fondation d'un Musée colonial et la création des cours didactiques: l'existence de l'une devant nécessairement impliquer la préexistence de l'autre (2). Enfin, pour tout

(1) Voici le titre de ces chaires: Io Histoire des produits végétaux coloniaux; $2^{\circ}$ Histoire des produits animaux et parasitologie des plantes et des animaux; 30 Minéralogie et géologie coloniale; $4^{\circ}$ Histoire et géographie coloniales; $5^{\circ}$ Législation et économie coloniales; $6^{\circ}$ Climatologie, épidémiologie et hygiene coloniales. Ces cours seront suivis par les élèves de la section coloniale de l'Ecole de commerce de Marseille, dès qu'elle aura été créée (octobre 1900.)

(2) 11 ne semble pas que cette vérité ait été bien comprise dans certains grand's centres manufacturiers de France, dont l'approvisionnementindustriel est dû à la production coloniale, comme Lyon, par exemple, où, sous l'empire des mêmes nécessités et à l'innitation de ce qui a été fait à Marseille bien antérieurement, des cours d'enseignement colonial ont été récemment inaugurés en dehors de toute préparation préalabledes éléments constitutifs d'un musée colonial. Il en est résulté tout naturellement que l'enseignement y a pris un caractère théorique qui ne saurait répondreaux besoins réels d'un auditoire surtout préoccupé des applications. Mais cet état de choses sera rapidement corrigé, quand, grâce aux ressources immenses que possède cette riche cité et aux relations qu'elle s'est déjà créées avec certaines de nos colonies où son influence commerciale s'affirme de jour en jour davantage. lę matériaux nécessaires à la création de cet instrument de premier ordre qu'on appelle un musée colonial, auront été réunis et classés. D'autre villes, comme Bordeaux et Nantes, s'occupent activement, sous l'impulsion de l'exemple marseillais, de procéder a ux mêmes créations, il était donc nécessaire de les mettre en garde contre l'écueil dans lequel est tombé l'enseignement de Lyon. 
épuiser dans cette matière, un troisième et tout exceptionnel mode d'enseignement, était offert par l'Exposition Universelle internationale de 1900. L'occasion était trop propice de montrer aux yeux du monde entier comment fonctionne et ce que produit cet instrument nouveau et très complet qui rapproche, sous le nom d'Institut et de Musée colonial de Marseille, un ensemble d'outillages groupés très harmonieusement vers un but commun, pour ne pas en profiter. Grâce à la bienveillance aussi éclairée que féconde de $M$. le Commissaire général J.-Charles Roux, envers qui le domaine colonial tout entier de la France a contracté une dette profonde de reconnaissance pour la haute intelligence et le profond dévouement avec lesquels il a pu, dans un laps de temps trop mesuré, mettre en évidence tout ce que nos colonies recèlent de plus intéressant, ce résultat a pu être obtenu. Le Musée colonial a exposé au pavillon du Ministère des Colonies et la présente notice a vu le jour. Nous ne saurions trop remercier ici M. J.-Charles Roux de l'intérêt qu’il a témoigné aux efforts d'un établissement qui ne demande qu'à affirmer de plus en plus son utilité, et qui atteindra ce but en s'inspirant de l'exemple que lui donne en cette circonstance solennelle le Commissaire général de l'Exposition coloniale de 1900, dont les hautes qualités organisatrices sont au-dessus de tout éloge.

Le Musée el l'Institut colonial de Marseille inaugurés en février 1896 par M. Nesureur, ministre du Commerce, furent fondés en janvier 1893 par M. le docteur Edouard Heckel, professeur à la Faculté cies sciences de Marseille et directeur du jardin botanique de cette ville. Une souscription recueillie par lui à domicile et dont le total s'élèva à 32. ooo francs, servit à former le premier fonds de création : le mobilier put être ainsi acquis et constitué par des vitrines à collection, corps de bibliothèque et un outillage de laboratoire de recherches. Les collections coloniales de tout ordre qui y figurent, eurent pour noyau les propres collections de M. Heckel; elles en ont formé le principal appoint. Depuis, grâce à son initiative, des dons personnels lui ont été adressés qui ont considérablement grossi le premier fonds. La bibliothèque d'abord réduite à un petit nombre de volumes apportés par $M$. Heckel et à quelques collections de 
périodiques intéressants, s'est grossie par le legs fait personnellement à $\mathrm{M}$. Heckel de la collection de livres coloniaux provenant de la succession Raoul, le légataire l'ayant reportée au Musée Colonial. En outre, toutes les publications coloniales périodiques intéressantes (journaux, rapports etc,) nous sont adressées par les diverses colonies qui ont intérêt à voir leurs productions connues. Le local a été fourni par le Ministère des colonies. Ce département a pris une grande part à cette création en réservant pour l'installation des collections tout le deuxième étage dans liimmeuble affecté à Marseille, 63, boulevard des Dames, au service colonial. En outre, une subvention annuelle de 5.400 francs a été consentie par le département pour le fonctionnement et l'entretien de cet établissement, tous les fonctionnaires qui y travaillent, en dehors du garçon de laboratoire qui remplit en même temps celle de gardien, (1 + foo francs de traitement), le faisant à titre purement gracieux. Il faut ajouter à ces ressources bien modestes, 250 francs votés annuellement par le Conseil général des Bouches-du-Rhône pour ce même Musée, ce qui porte les ressources totales et effectives du Musée à 4.050 francs par an (I). On verra bientòt à quelles multiples nécessités cette somme insuffisante doit faire face, pour assurer l'action très étendue de cet outillage nouveau dont le développement s“impose dans l’intérêt de la ville de Marseille, d'abord, de la science ensuite, enfin de la France entière qui jusqüici ne possède pas même à Paris, aucun autre établissement similaire (2). Nous dirons comment la ville de Marseille entend procéder à l'agrandissement de cet Institut-Musée colonial, et dans quelles proportions satisfaisantes elle se propose de le réaliser, pénétrée qu'elle est de la haute utilité de cette exten-

(1) Cesont là les ressources actuelles telles qu'elles ontété établies depuis un an, mais jusque là c'est-à-dire pendant six ans (1893 à 1898 ) elles étaient réduites à 1.250 fr. par an.

(2) Nous sommes bien loin sur ce point des autres nations colonisatrices qui depuis longtemps déjà ont organisé dans leurs capitales des musées coloniaux de premier ordre. Il suffit de rappeler que Londres possède le sien à la tête duquel se trouve, à titre de protecteur, le prince de Galles, ; que celui du Congo est à Bruxelle, (Tervueren) et a été fondé sous l'initiative et avec des ressources pécuniaires considérables fournies par le roi Léopold lui-mème, enfin que la Hollande possède un musée modèle 
sion pour assurer les destinées industrielles et commerciales de cette cité, actuellement plus orientée que jamais vers l'exploitation de nos colonies. Cette assemblée communale se montre ainsi soucieuse des hauts intérêts qui lui sont confiés et désireuse de maintenir, à l'encontre de la concurrence européenne qui la menace, à la ville de Marseille le titre bien mérité de Métropole de nos Colonies françaises. Il n'est pas douteux que, dans cette entreprise considérable, elle trouvera le concours du département et de l'Etat, qui n’ont pu, sans en être frappés, être témoins des efforts déjà réalisés par la municipalité en vue d'augmenter, dans un établissement d'enseignement supérieur qui relère de son budget. la somme des chaires coloniales nécessaires pour lui donner un caractère unique en France (1). C'est encore, grâce aux ressources de tout ordre accumulées dans l'Institut colonial de Marseille et à ses relations établies avec toutes nos colonies tant françaises qu'étrangères, que l'ensemble de cet enseignement médical pourra prendre le caractère matériel concret qui lui convient. Ces ressources ont été mises par M. Heckel

quoique restreint, à Haarlem, où sont entassées les richesses des Indes néerlandaises. Il ne faut pas oublier que l'Allemagne, dont la puissance coloniale est bien récente encore, a inauguré récemment un musée colonial à Berlin (1899) et que, l'empereur lui même, témoignant ainsi du haut intérêt que lui iaspirent les questions coloniales, a présidé, en personne accompagné de l'impératrice, cette solennité. Mais, il faut le dire aucun de ces établissements ne possède. comme celui de Marseille, un institut de recherches coloniales et d'enseignement annexé au musée.

(1) La ville de Marseille a voté en 1899 (2o juillet) une somme de ıo.00o fr. pour la création, auprès de l'Ecole de médecine de cette ville, de cing cours coloniaux dans I'ordre suivant: $1^{\circ}$ Clinique des maladies coloniales. $2^{\circ}$ Pathologie exotique et bactériologie. 30 Histoire naturelle et parasitologie coloniales, 50 Hygiène, épidémiologie, climatologie exotiques, 50 Bromatologie, matières médicales exotiques. Trois de ces cinq chaires sont pourvues actuellement de leurstitulaires et fonctionnent régulièrement. Les deux autres restent à pourvoir, mais il n'est pas douteux que le département de l'Instruction publique, à qui incombe le choix de ces titulaıres, ne tardera pas à répondre au désir manifesté si ouvertement par la municipalité, d’ouvrir aux connaissances coloniales de tout ordre, une place indépendante dans le haut enseignement de Marseille. Sur ce terrain d'intérêt général bien entendu. la municipalité et la chambre de commerce se sont rencontrées dans un effort commun qui ne manquera pas de porter ses fruits à brève échéance. 
sans réserve à la disposition de la municipalité de Marseille et des professeurs coloniaux, comme elles servent déjà à l'enseignement créé par la Chambre de Commerce.

Comme on le voit par ce court exposé, l'influence exercée par le Musée et l'Institut colonial de Marseille sur le développernent de l'enseignement colonial a été rapide, et, cet établissement entre luimême dans le concert didactique pour une grosse part en démontrant aux yeux, au moyen des collections et des Annales, combien la matière coloniale est riche et variée. Examinons maintenant en détail les organes divers qui forment cet appareil complexe et suivons en le fonctionnement. Nous montrerons ensuite avec quelques détails aussi, les résultats obtenus à longue ou courte échéance par le travail produit Nous avons vu le rayonnement d'influence de cet établissement scientifique, la façon dont, organe lui-même d'enseignement, il a permis le développement d'un cycle didactique complet à Marseille, suivons le dans son fonctionnement en prenant chaque organe à part. Nous verrons, après, ce que valent les oưriers bénéroles qui se sont chargés de la mise en mouvement de ces divers organes.

\section{MUSÉE COLONIAL}

Une vaste salle de 14 mètres de long sur 8 de large, située au deuxième étage de l'hôtel du service colonial à Marseille, est affectée à ce Musée. Dans cette salle d'exposition, la plupart des produits sont rangés par pays d'origine (toutes nos colonies étant représentées par leurs productions des trois règnes), dans de belles vitrines qui sont les unes horizontales, placées sur plusieurs rangs parallèles au milieu de la salle, et les autres verticales appliquées contre les murs. Au-dessous des premières, dont le plus grand nombre contiennent sous verre des produits de comparaison tirés des colonies étrangères (produits commerciaux), sont les herbiers dans des cartons et aussi de grosses pièces de géologie et de minéralogie qui n'ont pu trouver place, à cause de leur volume, sous les vitrines. Les objets volumineux tels que les manteaux, parasols, armes, costumes, canots, mannequins, instruments de musique (l'ethnographie en un mot), 
et les billots de bois, ou bien les produits trop volumineux, sont placés sur les parties des murs demeurées libres, entre les vitrines horizontales ou dans l'embrasure des fenêtres. Il a mème été nécessaire à cause de l'accroissement des collections, d'utiliser les couloirs et l'escalier. Dans les vitrines verticales, se trourent tous les produits végétaux présentant un intérêt scientifique ou économique. Beaucoup d'entre-eux sont nouveaux et ont reçu une application à

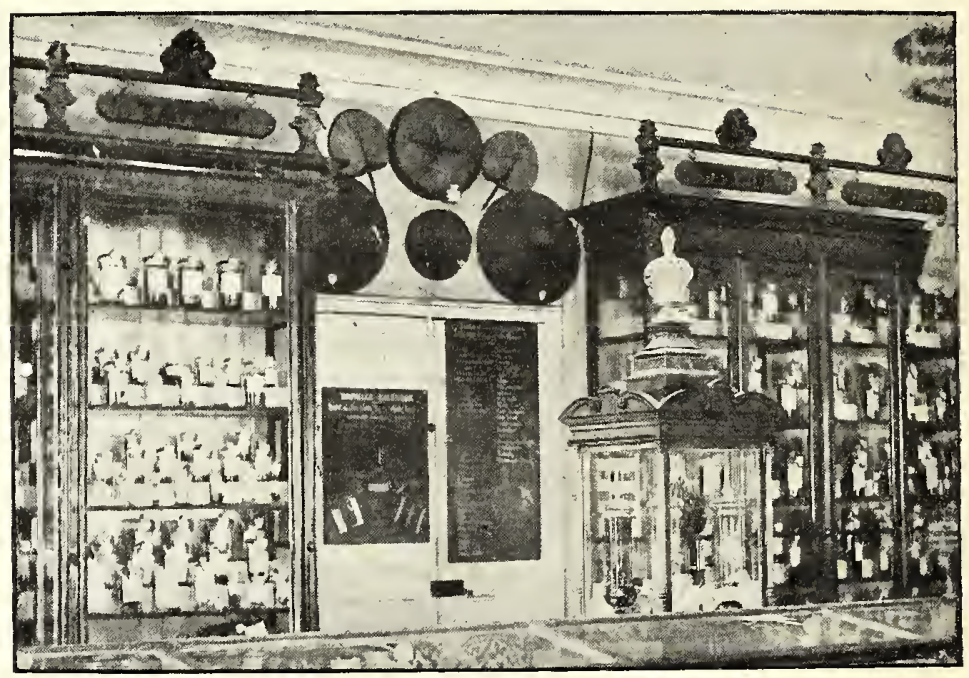

Vue de l'entrée de lo salle du Musée Colonial de Marseille

l'industrie ou au commerce soit local soit général, à la suite des études dont ils ont été l'objet dans les laboratoires de l'Institut colonial. Quand un de ces produits a reçu des applications, il figure en collection avec toutes les manipulations industrielles dont il est l'objet. Ainsi, les Araucarias divers de nos colonies s'étant montrés, 'd'après les recherches de $M$. Heckel, des producteurs de gomme résine dont la gomme est de l'arabine et la résine un produit comparable au Copal pour la fabrication des vernis gras ou des vernis à l'alcool, on voit à côté de la gomme-résine naturelle propre à chaque espèce, 
(1. Cooki, Rulei Bidwilli, imbricata, Brasiliensis, etc) la gomme, puis la résine et les vernis qu'elle donne. De même, chacune des graines grasses nouvelles et capables d'entrer dans l'industrie soit de la stéarinerie, soit de la savonnerie d'après les recherches récentes de M. Heckel, présente en série, à côté du produit naturel, le corps gras, les acides gras de distillation, de saponification, la glycérine et les bougies ou les savons qu'il peut donner; de même, enfin, un

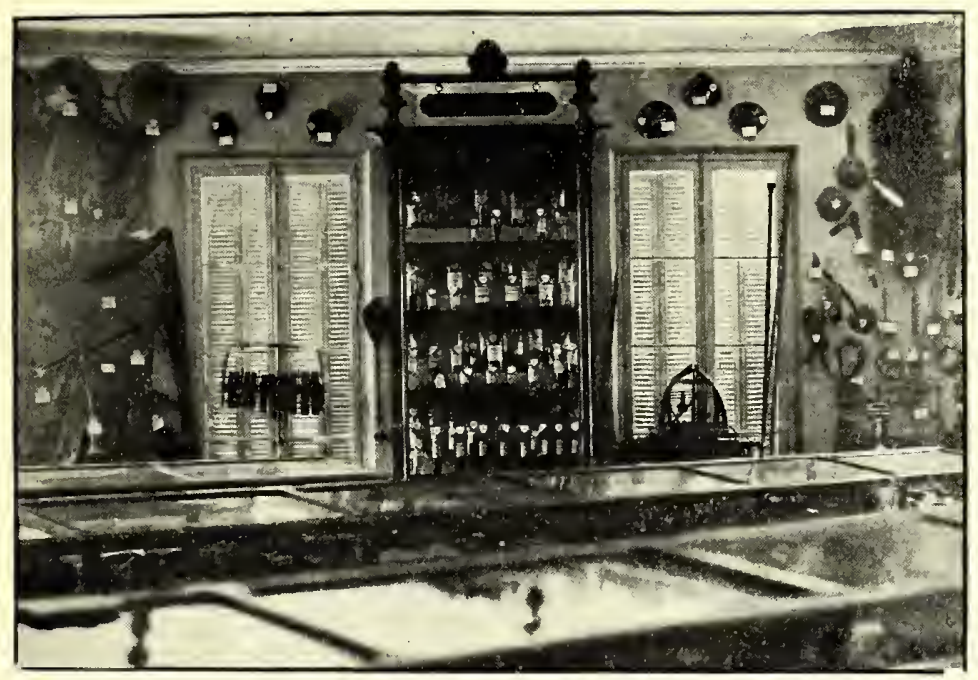

Vue du fond de la salle du Musée Colonial dr Marseille

produit naturel d'ordre médicinal se montre en nature accompagné des alcaloïdes, des glucosides ou des principes actifs divers qui entrent dans sa composition et lui donnent son utilité thérapeutique. Un produit naturel féculent est accompagné de sa fécule et de sa teneur en ce principe nutritif; une écorce ou une racine ou une feuille colorante, de son principe colorant; une écorce tannante de son acide tannique ou gallique. C'est là une des caractéristiques propres à cet établissement scientifique. Les bois sont présentés sous leurs trois conditions, quand. ils sont susceptibles de servir à la 
menuiserie ou à l'ébénisterie : $\mathrm{I}^{\mathrm{o}} \mathrm{A}$ l'état brut, le plus souvent de forts billots de 1 mètre de haut représentant le tronc naturel, puis $2^{\circ}$ au sommet du tronc une coupe transversale donnant la section brute polie; enfin $3^{\circ}$ une deuxième section verticale est vernie. Les collections de bois de la Nouvelle-Calédonie (très riche); de la Côte d'lvoire et celles de la Réunion se présentent dans ces conditions. On peut ainsi, à la fois, reconnaitre l'aspect normal du tronc, et apprécier la beauté plus ou moins grande du bois (1).

Plusieurs missions spéciales en Afrique, en Amérique et en Océanie, confiées à M. Jeanneney (Nouvelle-Calédonie), Dr Rançon (Haute Gambie), et Geoffroy (Guyane française), ont enrichi les collections d'une manière toute spéciale. Les herbiers comprennent des plantes de la Guyane (Bassières), de la Martinique et Guadeloupe (R. P. Duss), de la Réunion ( $\mathrm{D}^{\mathrm{r}} \mathrm{Jacob}$ de Cordemoy, père), du háut Tonking (Dr Simon), du haut Laos (Dupuy), de la Nouvelle-Calédonie (Heckel et Jeanneney), d'Australie (Meyden), enfin du Cambodge (Collard).

Le Musée de Marseille compte aujourd'hui des correspondants dans toutes nos possessions. Médecins, pharmaciens, administrateurs coloniaux et officiers de diverses armes en particulier, ont apporté, - avec un empressement qui prouve l'utilité d'une œuṿre appréciée dans ses résultats, - les collections qu'ils ont pu réunir dans leurs moments de loisirs; beaucoup d'industriels et de négociants sont venus exposer leurs produits et offrir, en même temps, des matériaux divers, récoltés incidemment au cours de leurs voyages coloniaux. Nous donnerons plus loin la liste complète de nos correspondants coloniaux et des donateurs de ce Musée. Les produits animaux les plus importants, en beaux échantillons, remplissent à peu près une vitrine: les produits minéraux très richement représentés par nos principales colonies, tiennent deux vitrines: les collections d'Algérie et de la Nouvelle Calédonie sont les mieux représentées dans l'ordre minéral.

(I) A chaque espèce de bois correspond dans l'herbier un échantillon des autres parties de la plante (feuilles, fleurs, fruits). 
Toutes ces collections sont rigoureusement classées et déterminées; elles portent toutes des étiquettes très apparentes indiquant le nom indigène, la famille botanique, le lieu d'origine, le nom scientifique et l'emploi. Le travail de classification et de détermination est réparti entre tous les collaborateurs du Musée et de l'Institut colonial; l'entretien de ces collections, dont les doubles sont conservées dans des casiers spéciaux et dans un local réservé,

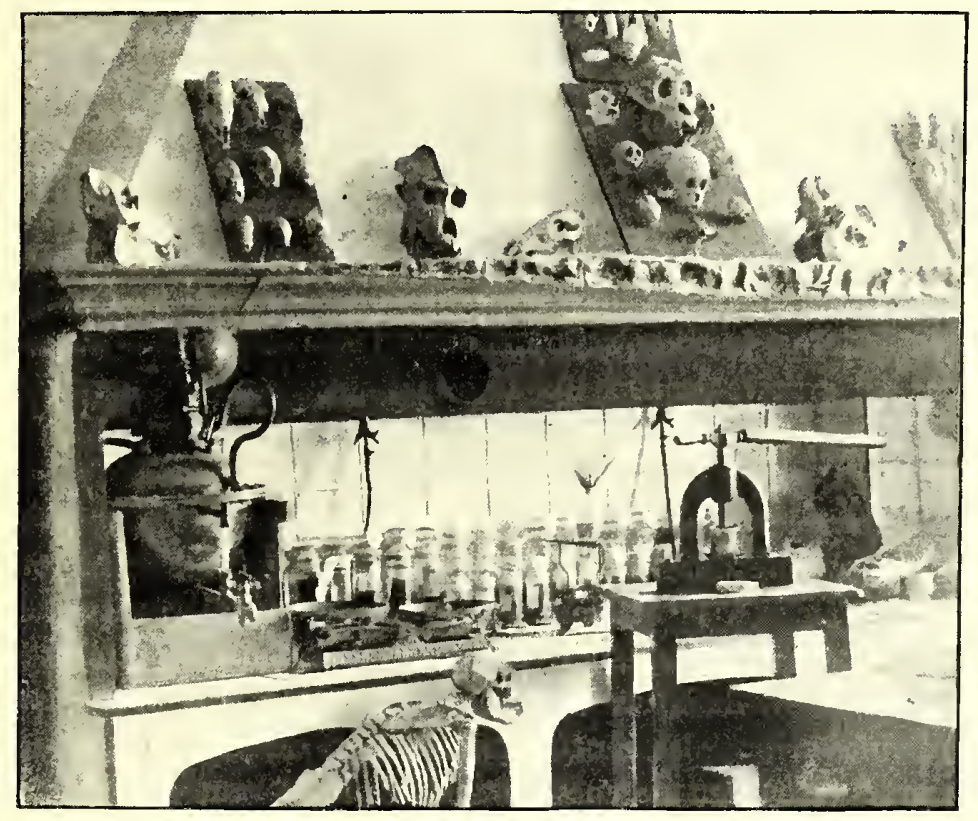

Vue d'une partie du laboratoire du Musér et de l'Institut Colonial de Marseille

et l'étiquetage sońt le lot de M. Eberlin, conservateur bénévole autant que dévoué. Un double catalogue par colonie et par ordre alphabétique a été dressé et est tenu à jour par M. Eberlin; il est à l'état de manuscrit mais il sera imprimé ultérieurement. Les doubles sont catalogués à l'état de fiches. Ces collections qui forment le Musée sont visitées régulièrement et assidument par le public auquel elles sont ouvertes tous les jours, le lundi excepté, de 9 heures à 
midi et de 2 à 5 heures. Un gardien à qui, un logement est 'réservé à côté du Musée au même étage, fait face à ce service et se tient à la disposition du public en meme temps qu'il s'occupe de la propreté du laboratoire, de la bibliothèque et de la salle d'Exposition. Le nombre des visiteurs pendant l'année 1899 a été de 20 par jour en moyenne.

A côté du Musée se trouve une salle de bibliothèque dont les volumes sont confiés également aux soins de $M$. Eberlin conservateur du Musée (1) cette salle aussi bien que le laboratoire sont fermés au public : elle sert de cabinet de travail aux collaborateurs de l'Institut colonial, soit pour la préparation de leurs leçons soit pour consulter la bibliothèque dans leurs travaux. Un catalogue existe.

Cette bibliothèque renferme le legs Raoul (livres coloniaux deculture et de botanique), et le legs Madinier, riche et très intéressante collection de dossiers relatifs à toutes les questions qui touchent de près ou de loin à la colonisation. Toutes les matières traitées sont réunies en articles de journaux découpés aux ciseaux. Cette collection de dossiers présente un grand intérêt rétrospectit, mais elle n'est évidemment plus à jour : néanmoins elle rend encore de grands services pour les documents historiques coloniaux de tout ordre. Toutes les publications coloniales officielles ou privées et les journaux sont reçus à la bibliothèque et y sont conservés avec les cartes géographiques coloniales qui sont toutes entoilées et classées méthodiquement dans un meuble spécial. Des photographies de nos colonies principales sont rassemblées en deux beaux albums et les murs recouverts de vues coloniales ou de portraits d'explorateurs coloniaux.

Le laboratoire sert à la fois à la manipulation, au classement des produits et à des recherches microscopiques ou physico-chimiques touchant leur composition. L'outillage n'y est pas très compliqué,

(1) Il est de toute justice de déclarer ici que, la première organisation et la première mise en ordre de cette bibliothéque sont dues à l'obligeance et aux services désintéressés de M. Fleury, actuellement bibliothécaire universitaire à Aix et alors (1894) bibliothécaire de la faculté des sciences de Marseille. 
mais des recherches plus délicates se poursuivent dans des laboratoires de chimie spéciaux. Au Musée se dégrossissent les recherches : on y prend une première impression de la valeur des produits et le reste s'achève ailleurs.

Citons maintenant les principales collections originales et les principaux produits nouveaux sur lesquels l'attention se trouvera attirée au cours d'une visite rapide. Nous commencerons par nos colonies africaines.

\section{COLLECTION DES PRODUITS D'AFRIQUE.}

Nos possessions de la côte ocsidentale, Sénégal, Soudan français, Guinée française et Dahomey, sont représentées par de nombreux produits, dont presque tous sont aujourd'hui déjà bien connus, grâce aux travaux de $M$. Heckel : quelques-uns sont entrés dans le commerce ou dans l'industrie et y tiennent une grande place.

Le Karité (Bassia Parkii), dont les graines fournissent un beurre de bonne qualité, et du tronc duquel s'écoule, en abondance, un latex qui, coagulé,pourra peut-être suppléer dans une certaine mesure la véritable gutta de plus en plus rare;

Le Maloukang ou Inkalaki dont les graines grasses, d'un grand avenir, fournies par le Polygala butyracea d'Heckel et étudiées avec soin par lui, sont l'objet de l'acclimatation actuelle dans nos colonies tropicales et donnent une graisse comparable pour le goùt au beurre de vache.

La noix de Kola (Cola acuminata), devenue d'un usage courant et dont l'histoire a fait l'objet du premier volume des Annales de l'Institut Colonial de Marseille dù entièrement à M. Heckel; elle tient actuellement une grande place dans le commerce de l'Europe.

Le Séribéli (Connarus africanus), dont les graines riches en tannin, ont des propriétés vermituges signalées à M. Heckel par le Dr Drevon, médecin principal des colonies; elles ont été bien étudiées par le Directeur du Musée Colonial dans les Annales de la faculté des Sciences de Marseille (en 1893).

Le Salikounda (Copaifera Salikounda), dont les graines sèches à odeur de coumarine, employées sous forme de pommade par les Sousous, pourraient passer pour un succédané de la Fève Tonka, bien 
que, d'après les recherches de $\mathrm{M}$. Heckel, celles-ci contiennent dixsept à dix-huit fois plus de principe aromatique (coumarine);

Le Dadigogo ou Balancounfa (Ceratanthera Beaumet $i$ Heckel), Scitaminée du Sénégal, tænifuge et purgatif par ses rhizomes frais. et sa tige :

Le Kinkélibah (Combretum Raimbanlti Heckel), qui, par ses teuilles employées en décoction, est le spécifique le plus en faveur contre la fièyre bilieuse hématurique, et que $M$. le $\mathrm{D}^{\mathrm{r}}$ Rançon a employé comme tel avec tant de succès, pendant la dernière expédition de Madagascar. Le succès de cette plante mise en honneur scientifique par $M$. Heckel s'affirme de jour en jour.

Le Néré ou Café du Soudan (Parkia biglobosa), dont les indigènes consomment, en nature ou en boisson fermentée, la pulpe sucrée des gousses, et dont les graines, torréfiées ou non, servent à faire une infusion théiforme et un condiment apprécié dans le Soudan; aliment spécialisé pour les enfants en France.

Le Doundaké (Sarcocephalus esculentus) que les propriétés fébrifuges et astringentes de son écorce ont fait dénommer le Quinquina africain; est dans le commerce de la droguerie française et des spécialités pharmaceutiques, depuis les travaux de M. Heckel.

Le M'Bentamaré ou Fedegosa (Cassia occidentalis), originaire d'Amérique, et probablement introduit sur la côte d'Afrique, mais qui est maintenant spontané, et dont toutes les parties sont employées, soit comme toniques, soit comme diurétiques, soit comme fébrifuges, - enfin dont les graines, dans le grand commerce, tendent, après torréfaction, à se substituer à notre chicorée indigène. Cette graine tient une grande place actuellement en Europe parmi les. succédanés du café depuis les travaux de M. Heckel.

A côté de tous ces échantillons (feuilles, graines, protuits), sont dans les mêmes vitrines, ou dans les vitrines voisines, les collections offertes par la maison Borelli et Mante frères, de Marseille : un régime de palmier oléifère (Elais guineensis), de l'huile de palme, des bandes de Raphia, des exemplaires de Voandzeia subterranea (l'arachide sauvage) provenant du Dahomey.

M. Bouëry, ingénieur établi en Guinée française, a exposé des 
caoutchoucs et des guttas et des troncs et feuilles des Ficus (Sakharé, Sakoui, Boro-boron-Khoré) qui les produisent, en même temps que des photographies de ces arbres, dont il poursuit la culture avec un soin et une intelligence louables.

Sur les rayons réservés au Sénégal, sont des arachides de Rufisque et Joal, des caoutchoucs, descires, des riz, de la gommecopal, donnés. par M. Bohn, le directeur si éclairé de la Société française de la Côte occidentale d'Afrique.

Hors vitrine, sont des balafons (1), et une série de bois de la Côte d'ivoire, recueillis par M. Pobéguin, administrateur colonial.

- Et à toutes ces collections viennent s'ajouter les documents de toutes sortes, (photographies, plantes, armes, etc.) rapportés en I 894, par le Dr Rançon, d'une mission au Soudan et en Haute Gambie. Le récit de cette mission, organisée par $M$. Heckel, et dans laquelle M. Rançon a découvert des peuplades nouvelles dans les pays des Bassarés et des Coniagués, et recueilli d'importants renseignements scientifiques et commerciaux, a été publié durant le deuxième volume des Annales du Musée Colonial de Marseille ( 1895 ).

En Guinée, il faut signaler spécialement les graines de Lamy Pentadesma butyracea) et de Méné (Lophira alata) étudiées avec grand soin par M. Heckel et qui promettent, en raison de leur abondance dans ces colonies, de leur rendement en corps gras et de la valeur de leur beurre, de devenir des produits très importants pour la stéarinerie, et même pour l'alimentation.

Au Gabon-Congo, nous retrouvons quelques-uns des produits précédents, mais on peut, entre autres, y signaler en plus :

L'O'Dika (Irvingia gabonensis), avec les graines duquel on prépare un pain gras très nutritif, d’une saveur analogue à celle du . cacao, et un beurre, moins cher que celui du cacao, qui peut être employé par la pharmacie et par l’industrie de la stéarinerie. Le

(I) Instrument de musique en bois fort remarquable et très en honneur sur toute la côte occidentale d'Afrique et dans le Soudan français. C'est le piano primitif, dont les cordes sont remplacées par des lattes en bois vibrant sous la percussion d'une baguette, armée d'une boule en caoutchouc. 
commerce s'en est emparé pour la préparation des chocolats à bon marché depuis les travaux de $M$. Heckel.

L'Owala (Pentaclethra macrophylla), dont les immenses gousses contiennent aussi des graines grasses. La maison Fournier, de Marseille, a fabriqué dernièrement, avec les corps gras de ces graines, des bougies à aspect de porcelaine, présentant l'avantage de fondre à une plus haute température que les bougies ordinaires, et, par suite coulant très peu. Ces bougies, ainsi que des savons et de la glycérine provenant du traitement de toutes ces graines, sont exposées au Musée. Cette graine, étudiée comme l'O'Dika, par M. Heckel, paraît être d'un grand avenir industriel.

Et, envoyés de la même région, citons encore : l'Iboga (Tabernanthe $I$ boga) dont les racines sont très recherchées des noirs qui s'en servent pour chasser le sommeil et comme excitant général; des caoutchoucs, des latex et des fragments de Landolphia qui ont été envoyés du Fernan-Vaz par M. Foret; différentes graines de Muscadier sauvage propres à donner des graines, le Poussah (Monodora myristica), graine à parfum suave, les graines et la gutta du N'Javé (Tieghemella africana), etc., tous produits pleins d'avenir.

Comparée aux colonies précédentes, notre grande île de Madagascar, possession nouvelle, est, pour le moment, un peu moins richement représentée. Cependant le visiteur pourra s'arrêter avec intérêt devant les échantillons de cristal de roche, de caoutchoucs, de vanille, provenant de différents donateurs, devant les rabanes de soie et de fibres végétales des maisons Ravel et Servel, devant les noix de Raventsara (1gatophyllum aromaticum) et les fruits de l'Arbre du voyageur (Ravenala madagascariensis) qui laissent entrevoir leurs graines à l'arille d'un beau bleu; et encore devant les régimes d'Hyphane et de s'agus, rapportés de Djaoudzi (Mayotte) par M. Castellan, lieutenant de port à Cayenne, dont le dévouement aux choses de la science est bien connu des naturalistes.

Les bois de Madagascar ont été très bien étudiés par M. Jumelle.

On pourra feuilleter aussi les premiers envois de plantes des environs de Majunga, jusqu'aux bords de la Betsiboka, préparées avec soin, sur la demande de $M$. Heckel, par le regretté $M$. Prince, 
pharmacien des colonies, mort victime de son zèle'dans cette colonie.

Quand à l'île voisine de La Réunion, les collections qui s'y rapportent sont, dès aujourd'hui, comme on peut s'y attendre, bien plus nombreuses. Elles sont principalement composées par des plantes en herbier, dons de M. Jacob de Cordemoy et de M. Creuze, et par des échantillons de quinquinas, de sucres, de cafés, de vanille, de paille de sousou (Sechium edule), de liqueurs, etc.

Les liqueurs (rhum, vin d'orange, vin dananas, etc.), sont exposées par la maison Vve Issautier, par le Crédit foncier colonial, par la Chambre de Commerce, etc.

La maison Ch. Issautier et fils a fourni des essences diverses (vétiver, géranium, basilic); la Sociéte de la rivière des Roches, du tapioca et du marioc; la maison Bousquet, de la vanille; la maison Julien Leyritz, des essences de géranium rosat, de citronnelle, de patchouly, etc...

Nous laissons de côté une foule d'autres produits d'origines diverses, dont beaucoup sont à l'étude; et, de l'Afrique, nous passons à nos colonies américaines : les Antilles françaises et la Guyane française, nos vieilles possessions les mieux connues.

\section{COLLECTION DES PRODUITS D'AMÉRIQUE.}

Dans la section de la Guadeloupe et de la Martinique, il faut citer en premier lieu l'herbier du R. P. Duss, dont la Flore des Antilles françai.jes a paru dans les Annales du Musée (année 1896) avec annotation du Prof. Heckel sur l'emploi de ces plantes.

Puis, comme échantillons commerciaux, nous remarquons surtout : des textiles, parmi lesquels le bois dentelle (Lagetta lintearia); du henné (Lawsonia inermis); l'indigo de l'Indigofera disperma, produit merveilleux, extrait de cette plante, introduite en I89o par M. Thierry, alors directeur du Jardin botanique de SaintPierre; du cacao en cabosses, de la farine de manioc, des graines de cacao, envois de diverses maisons (Rollin, Lafage, Wertier, Léon Dufour, Saint Wal-Noël, Laméol, etc.), des graines d'ambrette musquée (Abelmoschus moschiferus); du café Habitant de Delahongrais; du café Bonifieur, de la Chambre de Commerce de la 
Basse-Terre; d'autres variétés de café en parches et en grains données par la Chambre d'Agriculture de la Pointe-à-Pître; des cotons naturels; du sucre de canne de l'usine Souques; du bois de gaïac, du rhum de diverses provenances, adressé par la Chambre d'Agriculture de la Guadeloupe, grâce aux soins de son dévoué secrétaire général, M. Guesde.

A un point de vue plus purement scientifique, l'examen des vitrines de la Guyane française mérite une égale attention. Le Musée en effet, possède les plantes, minéraux et objets divers, rapportés en 1893 par le très regretté pharmacien des colonies Geoffroy, envoyé en mission sur le demande de M. Heckel.

M. Geoffroy, mort prématurément peu de temos après sa rentrée en France, avait exploré le Haut-Maroni, jusqu'au village des Roucouyennes. Il avait reconnu les ressources qu'offre en particulier le pays, au point de vue de l'exploitation de la gutta de Balata (Mimusops Balata); il avait recueilli aussi, chemin faisant, les matériaux d'un travail, qui a paru dans les Annales du Musée, sur le poison du Robinia Nicou, dont les indigènes se servent pour pêcher.

Et, à còté des collections de la mission Geoffroy, sont placés : les collections de bois de la Guyane, de M. Jeanneney; des guttas et des textiles, donnés par M. Hayes; du caoutchouc d'Hevea guyanensis; des substances économiques et médicinales variées; des corps gras et les graines qui les donnent, objet d'une étude actuelle de M. le Prof. Heckel.

N'oublions pas non plus ici une vingtaine de moulages représentant, en grandeur naturelle les chefs de tribus du Haut-Orénoque, du Rio Negro, du Meta, etc., aux curieuses coiffures, avec leurs attributs et leurs armes. Ces coiffures, ces attributs et ces armes, authentiques, ont été vendus 14,000 francs, autrefois, à la ville de Marseille, qui a obligeamment confié le tout au Musée colonial. Cette collection, d'une haute valeur, fait l'admiration de tous les hommes adonnés à l'étude de l'ethnographie. 


\section{COLLECTION DES PRODUITS D'OCÉANIE.}

En Océanie, la section de Tahiti nous présente: une importante collection de coquilles, ramassées par le Dr Buisson, médecin des colonies; des huîtres perlières; des produits médicinaux et économiques, envoyés par le $\mathrm{D}^{r}$ Vincent. Citons, par exemple, le KawaKawa (Piper methistıcum), aux propriétés multiples; le fameux cosmétique Monoï ; de la paille de bambou; des feuilles et des tubercules de Tacca; le coton longue soie hybride, dû aux soins dévoués de Raoul et qui fait prime sur les marchés européens.

Mais c'est devant la vitrine de la Nouvelle Calédonie que le risiteur s'arrètera le plus longtemps. Dès la fondation du Musée, cette colonie était une des mieux représentées, le Prof. Heckel, qui y a séjourné longtemps, ayant donné toutes les collections personnelles qu'il rapporta de cette île. Depuis lorš, le Musée, a reçu encore de nombreux envois de $M$. Jeanneney et du colonel Pelletier. Aussi, n'avons-nous, pour citer, que l'embarras du choix ; mentionnons:

Des blocs de résine et de gomme du Pin colonaire (1raucaria Cooki), qui trouvent leur emploi dans la fabrication des vernis gras et àl'alcool, aux lieu et place du copal : ce même produst donne une gomme appréciée ;

De la résine aromatique de Kaori (Dammara Moorei), fossile qui constitue le faux ambre et peut servir comme tel; de la gommerésine et des graines de l'. Lraucaria Biduilli, originaire d'Australie étudié par M. Heckel, et aujourd'hui planté avec succès en Nouvelle Calédonie, puis introduit par les soins de $\mathrm{M}$. Heckel dans notre colonie de la Réunion; les graines valent nos bonnes chataignes.

Des exsudations tanniques du Chène-gomme (Spermolepis tannifera Heckel), dont 11 . Heckel a fait connaitre la composition; de grand avenir industriel.

De la résine d'Érouma (Macaranga Vedeliana), étudiée récemment par M. Jumelle, et qui sert aux Canaques comme mastic d'une grande résistance;

Le caoutchouc du figuier de Calédonie (Urostigma prolixum) d'un excellent rendement et bien coté sur les marchés de Londres 
( 7 trancs le kilo), aujourd'hui exploité par une Compagnie française ;

Des feuilles et de l'essence de Niaouli (Melaleuca viridiflora), l'arbre caractéristique des terrains schisteux de la Nouvelle-Calédonie ;

Des graines et de l'huile de Tamanou (Calophyllam Inophyllum) et de Bancoul (Aleurites triloba), dont le corps gras reçoit des emplois industriels à Marseille ; dela résine très parfumée de Tamanou, et des blocs de résine de Gardenia si chère aux Canaques comme masticatoire.

Des coquilles diverses, etc. ; des essences merveilleuses (Myoporum, Beilschmiedia, Ocotea).

En outre, autour des vitrines horizontales, sont rangés des billots de bois variés, préparés et envoyés sur les ordres du colonel Pelletier. Ces billots, d'une hauteur de 1 mètre environ, sont fendus suivant le diamètre, sur une partie de leur longueur, et vernis sur la tranche. On peut ainsi, à la fois reconnaitrel'aspect normal du tronc, et apprécier la beauté plus ou moins grande du bois.

Ces échantillons desséchés viennent ainsi compléter en partie un autre herbier et des dessins donnés à M. Heckel par feu Jeanneney, agent de colonisation de l'Administration pénitentiaire, qui, en 1894, a publié un intéressant petit volume surla Nouvelle-Calédonie, guide, au point de vue agricole et botanique, plein de renseignements pratidues pour les émigrants. Il serait à désirer qu'un travail semblable et d'un caractère aussi consciencieux, fût fait pour toutes nos colonies françaises. (1)

\section{COLLECTION DES PRODUITS D'ASIE}

Ce coup d'œil rapide à travers le Musée se terminera par les colonies d'Asie.

Ici encore, le Musée colonial est redevable au colonel Pelletier de

(1) La Nouvelle-Calédonie agricole. Paris, Challamel, éditeur. Nous croyons savoir que l'Union coloniale française s'occupe de réaliser ce souhait. 
très nombreux objets. Tous proviennent du pays de Muong. Ce sont des poteries, des vases, des étoffes, des ‘vêtements, des instruments de musique, un canot et des reproductions en miniature des divers véhicules en usage dans le pays.

Pour le Laos, trois séries de collections ont été apportées: l'une par M. Rouet, inspecteur des postes, l'autre par M. Dupuis, commissaire du gouvernement et enfin le troisième par le capitaine de Bouillane de Lacoste.

A la générosité du premier, le Musée doit un lot important d'ustensiles employés dans le Bas-Laos; et M. Dupuy, qui administre le Haut-Laos, a fait don d'une riche collection d'objets en argent, (coffrets, vases. etc.). et d'étoffes laotiennes. La collection de bijoux du Yunam et du Haut-Tonkin de M. de Bouillane de Lacosteest d'une importance et d'une richesse exceptionnelles: elle figure à l'Exposition du Trocadéro (Musée colonial de Marseille).

Comme documents botaniques sur l'Indo-Chine, le Dr Simon, qui a séjourné dans le Haut-Tonkin, sur la frontière de Chine, a fait cadeau d'un volumineux herbier, accompagné de notes; feu Cabasse a donné des produits de matière médicale indigène du Tonkin; et le Service des Forèts envoie peu à peu des rondelles des divers bois du Tonkin et de l'Annam.

Ajoutons encore l'herbier du Haut-Laos récemment envoyé par M. Dupuy.

Au point de vue commercial, signalons spécialement plus de 50 variétés de riz de Cochinchine, des cafés, du cacao; du thé cultivé par M. Lombard, qui a établi des plantations en Annam; de la filasse de ramie (une vitrine est consacrée à la Société française «La Ramie » pour ses produits manufacturés).

\section{CLASSEMENT DES COLLECTIONS}

Et si, maintenant, après cette courte énumération, nous ajoutons que nous avons laissé de côté les collections minéralogiques de toutes les colonies citées - celle de Nouvelle-Calédonie et d'Algérie étant surtout très complètes -; si nous ajoutons que le Musée réunit 
aussi, comme types de comparaison, les produits similaires provenant des colonies étrangères (produits qu'il doit en grande partie à l'obligeance de la Compagnie des Docks et des Messageries maritimes) on se rendra compte de l'importance qu'a déjà prise le nouvel établissement.

Les visites qu'y rendent journellement les industriels et les commerçants marseillais sont, d'ailleurs, la meilleure preuve des services qu'ils en attendent et de l'intérêt qu'ils portent à cet établissement.

Les nombreux dons que nous avons signalés démontrent aussi que beaucoup de fonctionnaires coloniaux ont appris le chemin du Musée; un grand nombre ont contracté l'habitude de venir, avant leur départ, s'enquérir du genre de recherches quill y a surtout lieu de faire dans les contrées où ils vont résider. Les renseignements nécessaires leur sont fournis par M. Heckel et par les collaborateurs qui l'entourent.

Mais les services rendus par le Musée colonial ne sebornent paslà. La Chambre de Commerce de Marseille reçoit des quatre points du monde, à raison de la situation privilégiée de notre port (point de convergence de toutes lignes de paquebots reliant l'Europe aux colonies), des demandes de renseignements sur.la valeur industrielle et vénale d'une foule de produits exotiques, soit connus, soit inconnus dans le commerce et l'industrie. Grâce aux ressources offertes, soit par les collections commerciales et scientifiques, soit par la bibliographie, des réponses satisfaisantes peuvent être sur tousces pointstransmises rapidement aux intėressés pour le plus grand avantage du commerce marseillais. La Chambre de Commerce veut bien sur ce point s'en rapporter aux renseignements que lui fournit le Directeur du Musée colonial. D'autre part, le service des douanes se trouve souvent en présence de produits nouveaux arrivant dans lecommerce sous des noms indigènes ou mème fantaisistes qui ne la renseignent en aucune façon sur la nature et l'emploi probable de ces produits; de là pour cette adiministration l'impossibilité de pouvoir frapper un droit d'entrée par assimilation. Le Directeur du Musée colonial est alors consulté et l'assimilation peut être faite dans l'intérèt du Trésor. Enfin, les litiges commerciaux pour avaries de merpeuvent 
être résolus par le Trıbunal de commerce de Marseille, gràce à la consultation des types commerciaux dont la sincérité et l'origine sont indiscutables et indiscutés : il en est de même des procès intentés pour livraison de marchandises non conformes aux conventions; les types commerciaux du Musée colonial peuvent servir d'étalons dans ces divers cas.

\section{INSTITUT COLONIAL.}

Sous ce nom, il faut entendre un groupement d'instruments divers de travail et de chercheurs appelés à les mettre en mouvement dans l'unique but de contribuer à l'étude des produits nouveaux ou peu connus des colonies françaises. Ces organes sont: $I^{\circ}$ le Musée colonial dont nous avons déjà examiné l’ordonnance générale et les moyens, nous n'y reviendrons que pour dire qu'il est la source où s'approvisionnent les laboratoires de recherches. Il est inutile d'insister sur ce rôle. $2^{\circ}$ La bibliothèque qui récèle les documents bibliographiques et les publications capables de renseigner sur les produits mis en étude; enfin : $3^{\circ}$ le jardin botanique colonial avec son laboratoire annexe qui constitue un instrument important d'études dont nous n'avons pas parlé jusqu'ici. Cet établissement appartient à la municipalitéde Marseille et n'est qu'une dépendance du jardin botanique de la ville placé sous la direction de $M$. le professeur Heckel au parc Borély. S'agit-il de connaître la valeur d'une graine quelconque arrivée à l'état frais et sans aucun document botanique, par exemple, et faut-il faire l'étude du végétal qui la produit? On la met en germination dans des serres chaudes spéciales affectées aux plantes coloniales et généralement elle arrive assez rapidement à parcourir ses phases végétatives. Il en est de même des bulbes, oignons, tubercules, éclats, rhizomes, etc., qui nous sont envoyés des colonies sans la plante mère. C'est ainsi que la détermination du Dadigogo (Ceratanthera Beaumetzi Heckel), tænifuge de la Guinée, a pu être possible après floraison de la plante dans les serres du jardin botanique colonial. Certaines plantes (grands végétaux) n’arrivent pas à floraison, mais se développent assez pour que la détermination en soit possible sur l'aspect général, après consultation des flores locales, et des herbiers du Musée. Ces germinations rendent du reste de très grands services en permettant a uxobservateurs de décrire les phases 
de première végétation qui ont échappé le plus souvent aux purs descripteurs des plantes qui en naissent. L'histoire des végétaux tropicaux se complète très utilement de cette façon et leur biologie devient plus connue. M. Heckel a découvert ainsi le parasitisme des Olacinées.

Le laboratoire de micrographie annexé à ce jardin botanique comprend, en outre, une petite bibliothèque botanique coloniale et une salle de recherches chimiques outillée assez commodément pour déterminer, par exemple, la composition d'une gutta, ou d'une graine grasse, d'un caoutchouc, d'une résine, d'une gomme résine, d'une plante à tannin. Les recherches de cet ordre commencées au laboratoire du Musée colonial s'achèvent là. M. le protesseur Jumelle a pu mener à bien avec ces ressources, ses belles et délicates recherches consignées dans son livre sur les Caoutchouc et les gutta, et, dans cette notice, celles également qu'il a faites sur les cacaos et les réactions colorées que présente la graine, suivant ses origines, sortent de ce laboratoire. Un autre centre de recherches chimiques se trouve au laboratoire botanique de la faculté des Sciences et à l'Ecole de Médecine où ont été réalisées les nouvelles données sur les gommes et sur les résines réunies dans le livre récent de $M$. Jacob de Cordemoy sur les produits de nos colonies françaises (1). Le travail de recherches qui doit porter sur les produits de tout ordre, c'est-à-dire appartenant aux trois règnes de la nature, a été jusquici plus particulièrement orienté vers les matières premières végétales qui sont les plu^ nombreuses, les plus variées et les plus intéressantes sans conteste. Mais la spécialisation didactique des deux professeurs de produits animaux coloniaux (M.J. de Cordemoy) et de produits minéraux (M. Lau-

(1) Des travaux de chimie importants ont été également faits sur les produits végétadx économiques des colonies françaises dans le laboratoire de chimie de l'Ecole supérieure de pharmacie de Nancy, par M. le professeur Schagdenhauffen, collaborateur habituel de M. Heckel depuis plus de 25 ans que dure leur association fructueuse pour la science. La plupart des travaux coloniaux de ces deux professeurs ont été faits en collaboration et il serait difficile de les énumérer tous ici sans omission. La liste approchée en sera donnée à la fin de cette notice. Quelques élèves ou maitres des laboratoires d'autres universités (Nancy, Clermont-Ferrand, Toulouse, Montpellier) ont fourni aussi des travaux aux Annales après avoir reçu du Musée colonial la matière première de leurs recherches. 


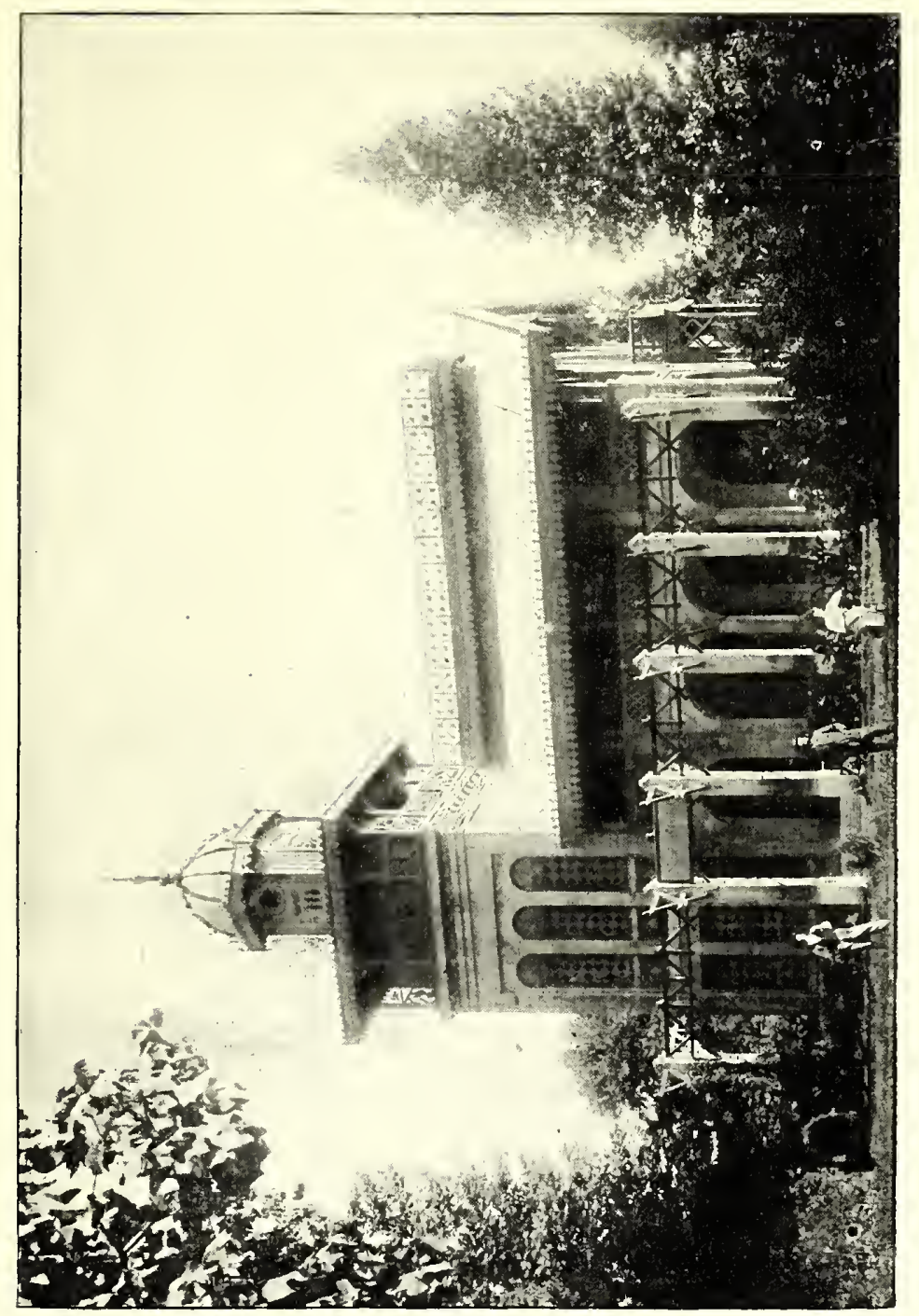

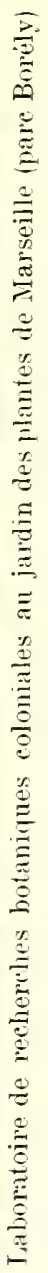


rent), nous promet pour l'avenir la mise en ouvre d'autres études dont l'intérêt n'est pas à démontrer.

Quoi qu'il en soit, tous les travaux sortis de ces laboratoires divers et dus aux collaborateurs dont les noms suivent, ont étẻ insérés dans une publication annuelle connue sous le nom d'Annales de l'Institut colonial de Marseille et qui existe depuis 1893 sans interruption. Nous allons parler de cet organe dont la place dans la littérature scientifique est faite aujourd'hui, mais disons d'abord qu'elle a été jusqu'ici alimentée par les travaux de MM. le professeur Dr Heckel, directeur-fondateur du Musée colonial; Jumelle, professeur-adjoint à la Faculté des Sciences; Jacob de Cordemoy, chef des travaux botaniques à la Faculté des Sciences et chargé d'un cours colonial à la Chambre de Commerce. Antérieurement à 1893 , M. le professeur Heckel dont les publications sur les produits végétaux économiques de nos colonies françaises datent d'avant 1870 , consignait ses recherches dans divers périodiques de la capitale (Journal de Pharmacie et de Chimie, Journal de Thérapeutique, Journal La Nature, Revue Scientifique, Revue générale des Sciences, Répertoire de Pharmacie, Dictionnaire Encyclopédique des Sciences, Archuves de Médecine navale, Dictionnaire des Sciences Médicales de Dechambre, Revue des Cultures coloniales, Annales de la Faculté des Sciences de Marseille, (I), etc.). Depuis la création des 1 nnales de l'Institnt colonial qu'il a réalisée en 1893 à la même époque que son Musée colonial, ses principaux travaux originaux, comme tous ceux de ses collaborateurs sont réservés pour cette publication périodique qui paraît en un volume annuel de

(I) Cinq importants travaux coloniaux de M. Heckel sont à signaler dans ces Annales savoir: Etude sur la flore de la baie de Prony (Nouvelle Calédonie) avec une carte et des figures dans le texte (1802); Sur la germination de 'A raucaria Bidwilli $2^{\circ}$. Monographie sur le Copahifera Salikounda Heckel, de la côte occidentale d’Afrique et sur sa graine à Coumarine (เ 1893$)$ 3o Monographie sur le Séribéli (connarus africanus Afz.) tœnifuge de la côte occidentale d'Afrique (1897), ces deux monographies avec planches en couleur ; $4^{\circ}$ Contribution à l'étude botanique de quelques Solanum tubérifères (1898). 50 Sur le Dadi-Gogo ou Balancounfa (Caratanthera Beaumet $\{\mathrm{H}$ Heckel). plante nouvelle dont le rhizome est employé comme tœnifuge sur la côte occidentale d'Afrique (1891) avec trois planches dont une en couleurs (5o pages). 
500 pages environ avec figures ou cartes dans le texte ou hors texte. Cette collection est imprimée par M. Protat, à Mâcon. Ces Annales subventionnées par le Conseil général des Bouches-du-Rhône ( $500 \mathrm{fr}$. par an), sont tirées à 300 exemplaires et adressées à titre d'échanges aux Universités françaises et étrangères, ainsi qu'aux établissements coloniaux similaires dans le monde entier. Sept volumes des Annales ont paru de 1893 à 1900 exclus. Voicile sommaire des travaux qui y sont insérés avec le nom de leurs auteurs:

$I^{\text {re }}$ année 1893 . - $1^{\circ}$ Les Kolas africains au point de vue botanique, chimique, commercial et bromatologique; $2^{\circ}$ Des beurres de Dika et de Cay-cay et des plantes qui les produisent au Gabon et en Cochinchine par MM. Heckel er Schlagdenhauffen (avec figures en noir, en couleur dans le texte et hors texte).

$2^{\mathrm{e}}$ année 1894. - Voyage d'exploration scientifique en Haute Gambie par le Dr Rançon, médecin principal des colonies (avec figures, dans le texte et hors texte).

$3^{\text {e }}$ année I895.- Sur le Robinia Nicou de la Guyane (liane à enivrer le poisson), par E. Geoftroy; $2^{\circ}$ Contribution à l'histoire botanique, chimique et thérapeutique des Idansonia, par le DrGerber; $3^{\circ}$ Le Quassia africana (botanique, chimie et thérapeutique), par le Dr Claudel; $4^{\circ}$ Sur le Bakis et le Sangol du Soudan par E. Heckel et Schlagdenhauffen; $5^{\circ}$ Exude sur le Goyavier, par Khouri, pharmacien de Ire classe de Paris.

4 année I 896 . - Flore phanérogamique des Antilles françaises, par le R. P. Duss (avec annotation du professeur Heckel sur l'emploi de ces plantes), I volume de 656 pages.

$5^{\circ}$ année 1897 . - $1^{\circ}$ Rapport de mission scientifique à la Martinique et à la Guyane, par E. Geoffroy; $2^{\circ}$ Les plantes médicinales et toxiques de la Guyane française, par E. Heckel; 30 Recherches sur les graines grasses nouvelles et peu connues des colonies françaises, par E. Heckel; $4^{\circ}$ Sur un Strophantus du Congo français (Strophantus d'Autran), par Schlagdenhauffen et Louis Planchon; $5^{\circ}$ L'Erouma de Nouvelle-Calédonie et sa résine, par H. Jumelle; $6^{\circ}$ De l'écorce fébrifuge du bois piquant (Zanthoxylum Perrotetii 
D C.), par E. Heckel et Schlagdenhauffen; $7^{\circ}$ Sur les Murraya de Cochinchine, par le Dr Laborde, de Toulouse.

$6^{\mathrm{e}}$ année 1898 . - $\mathrm{I}^{0}$ Les plantes à caoutchouc et à gutta des colonies françaises, par H. Jumelle; $2^{\circ}$ Les graines grasses nouvelles ou peu connues des colonies françaises, par M. E. Heckel; $3^{\circ} \mathrm{Un}$ nouveau Jaborandi des Antilles (Pilocarpus racemosus Wahl), par le professeur Dr Rocher, de Clermont-Ferrand.

$7^{\mathrm{e}}$ année I 899. - $\mathrm{I}^{\circ}$ Le Cacaoyier, sa culture et son exploitation dans les colonies françaises, par H. Jumelle; $2^{\circ}$ Gommes, résines et gommes-résines des colonies françaises avec les végétaux qui les produisent par le $\mathrm{D}^{\mathrm{r}} \mathrm{J}$. de Cordemoy.

En 1900 paraîtront, avec une vaste étude sur le tabac par M. Laurent, des travaux de zoologie sur les Grillidés et les Acridiens qui causent des ravages considérables dans nos cultures coloniales, par M. le Dr Bordas de la Faculté des Sciences de Marseille. Un résultat important se dégage, au point de rue des intérêts commerciaux et industriels de Marseille, c'est qu'en dehors des faits acquis à la science pure par les études ci-dessus mentionnées, un certain nombre de produits végétaux de nos colonies françaises, ont pu après examen scientifique approfondi, être tirés de leur inutilisation et se sont créé, grâce à ces études, une place soit dans le commerce, soit dans l'industrie de la France, rapportant ainsi à la métropole les sacrifices, légers du reste, qu'elle s'impose pour l'entretien du Musée colonial de Marseille et de son Institut, ce sont :

$1^{\circ}$ Les Kolas africains dont le commerce et la manipulation manufacturière en France sont très importants; $2^{\circ}$ les graines de M'bentamaré (Cassia occidentalis L.) qui se substituent de jour en jour à la chicorée comme succédané du café et arrivent en grande quantité notamment à Bordeaux et à Marseille; 3o L'Owala et le Lamy, graines grasses qui prennent une place marquée dans l'industrie de la stéarinerie, avec la graine de Karité qui donne aussi une bonne huile concrète et est employée pour être mélangée en nature à la graine de cacao, dans les fabrications des chocolats inférieurs; $4^{0}$ le Pilocarpus racemosus des Antilles françaises (bois flambeau) qui remplacera bientôt le Jaborandi du Brésil dans les 
drogueries; le beurre et la graine de Dika du Gabon appelés à remplacer à bas prix le beurre de cacao et déjà utilisés en Espagne; $5^{\circ}$ le Kinkélibah (Combretum Raimbaultii Heckel), très demandé et qui entre franchement dans le commerce de la droguerie comme remède très efficace contre la fièvre bilieuse hématurique; $6^{\circ}$ la Tannorésine de Spermolepis tannifera Heckel, de Nouvelle-Calédonie, très utile pour les tanneurs à cause de sa richesse exceptionnelle en acide tannique; $7^{\circ}$ la Gutta de Karité (Butyrospermum Parkii), du Soudan français, demandée en Angleterre et utilisée comme succédané de la vraie gutta des îles de la Sonde; la Résine fossile de Kaori (de Nouvelle-Calédonie) mise en œuvre pour remplacer l'ambre vraie dont elle a l'aspect et quelques propriétés; $9^{\circ}$ la Gomme de M beppe du Sénégal-Soudan, qui est appelée à jouer un rôle comme gomme arabique à bon marché; $10^{n}$ les gommes-résines des Araucarias (Nouvelle-Calédonie), sources de gomme arabique industrielle et d'une résine qui peut remplacer avantageusement et à moins de frais, la gomme copal pour la fabrication des vernis gras et à l'alcool. Voilà quelques résultats qui semblent promettre mieux encore pour l'avenir, quand l'outillage du Musée et de l'lnstitut colonial aura été rendu plus parfait et ses ressources mieux assurées qu'à l'heure actuelle où son budget est absolument dérisoire et où son existence n'est réalisée que par des prodiges sans cesse renouvelés. 


\section{NOUVEL INSTITUT COLONIAL \\ PROJETÉ PAR LA MUNICIPALITÉ DE MARSEILLE}

Tout ce qui précède est relatif à l'état actuel du Musée et de l'Institut colonial de Marseille, examinons ce qu'il semble appelé à devenir dans un avenir prochain. En I 897, M. Eberlin, conservateur du Musée colonial de Marseille, et très précieux collaborateur, faisait connaitre aux lecteurs de la Reyue générale des Sciences ( $\mathrm{N}^{\circ} \mathrm{du}$ 15 novembre 1897) l'historique et l'état de cet établissement d'enseignement supérieur au moment où il écrivait. Depuis cette époque, les collections tant scientifiques que commerciales se sont enrichies à ce point que les vitrines et les armoires dédoublées débordant de tout côté, il a fallu songer à demander aux corps élus une place convenable pour abriter ces richesses de toute nature et pour en faire profiter l'enseignement, la science, l'industrie et le commerce. La municipalité Marseillaise, obéissant à un mouvement aussi généreux qu'éclairé, a voté un crédit de 450.000 francs (I) pour la construction d'un Musée-Institut colonial à bâtir sur un terrain de même valeur situé au cœur nouveau de la vilie dans un quartier (ancien cimetière Saint-Charles) réservé à l'édification des bâtiments qui doivent constituer l'ensemble des facultés dont Marseille sera dotée un jour ou l'autre, c'est-à-dire dans la partie la plus déclive du boulevard de la Paix, avoisinant la place d'Aix.

Cet emplacement situé à égale distance des quartiers les plus peuplés, est bien le centre futur de la ville de Marseille. 11 est peu distant de la Joliette, lieu de débarquement et d'embarquement de la multitude des voyageurs d'outre-mer qui arrivent chaque jour dans. notre ville, soit de provenance soit avec une destination coloniale. Ceux-ci pourront donc, sans effort, visiter le Musée colonial en remontant le boulevard des Dames qui aboutit à la place d'Aix, centre

(1) Cette somme est comprise, avec une autre de 30.000 fr. destinée à l'édification d'une serre coloniale au jardin botanique, dans un emprunt qui doit être soumis au vote des Chambres. Le vote municipal a eu lieu le 28 juin 1898 . 


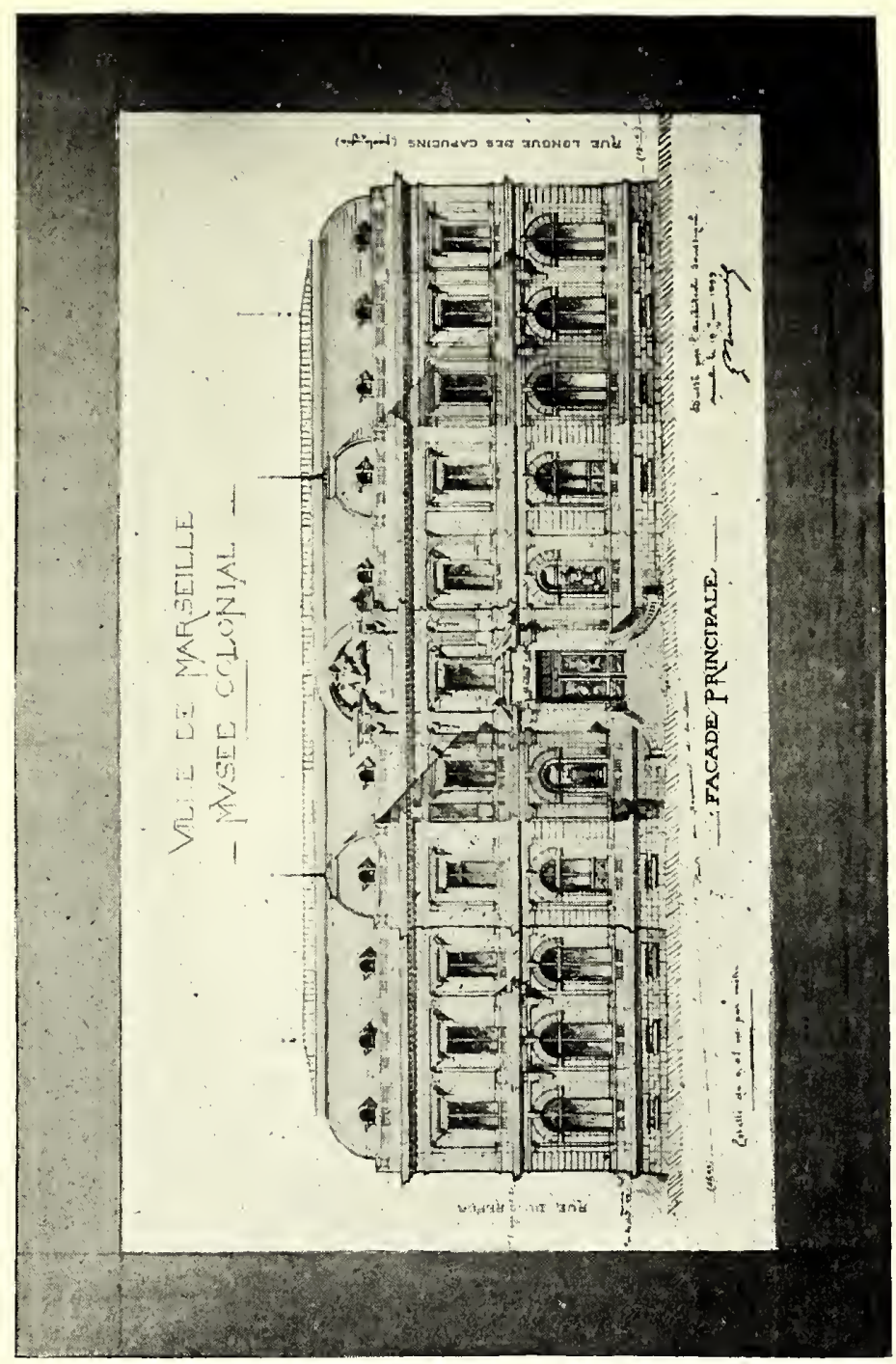

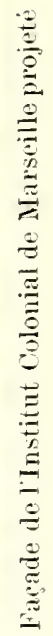


de la ville, et qui sera prochainement desservi par une ligne de tramway reliant directement le port à la gare de Marseille.

Avec son double caractère de Musée et d'Institut de recherches coloniales, cet établissement devant être, quand il sera construit, le seul de ce genre qui existera en France, il y a certainement intérêt général, à un moment où, voulant suivre l'exemple donné par Marseille, les villes commerciales et industrielles de notre littoral océanien, comme Bordeaux et Nantes, ou même de l'intérieur, comme Lyon, se disputent à l'envi la priorité d'une organisation de Musées coloniaux, à faire connaître les dispositions qu'a cru devoir adopter M. Heckel, pour mettre cette création nouvelle en harmonie avec la science moderne et les services qu'elle doit rendre à nos colonies françaises. Il convient aussi, pour l'intelligence du texte, d'accompagner cette description des divers plans, réduction de ceux qui ont été établis, sur les indications de M. Heckel, par M. Mouriès, architecte marseillais du plus grand mérite.

Pour les dispositions intérieures, M. Heckel ne s'est inspiré que des besoins actuels de nos colonies et du commerce métropolitain. C'est à dessein qu'il a négligé de se servir des données à emprunter aux établissements similaires de Haarlem, de Bruxelles et de Londres: ces créations sont propres à des nations colonisatrices plus avancées que nous dans leur cuvre ou qui, disposant de moyens spéciaux et d'un empire colonial différent du nôtre, ont pu avoir de leurs nécessités coloniales un concept tout différent de celui de la France. Il ne faut pas se dissimuler, que, malgré l'expérience acquise par la création de toute pièce d'un Musée restreint, qui a toujours été considéré comme la maquette de celui dont la réalisation semble prochaine pour Marseille, combien cette œuvre sera imparfaite par divers côtés. Le temps permettra de l'améliorer dans l'avenir, par des modifications de détails. Ceci dit, voici le dispositif du monument dans son ensemble.

Le nouveau Musée colonial contiendra quatre grandes salles de collections affectées, avec des proportions différentes, à nos colonies d'Afrique, d'Amérique, d'Océanie et d'Asie. Les collections déjà existantes, s'augmenteront encore de tous les produits peu connus 


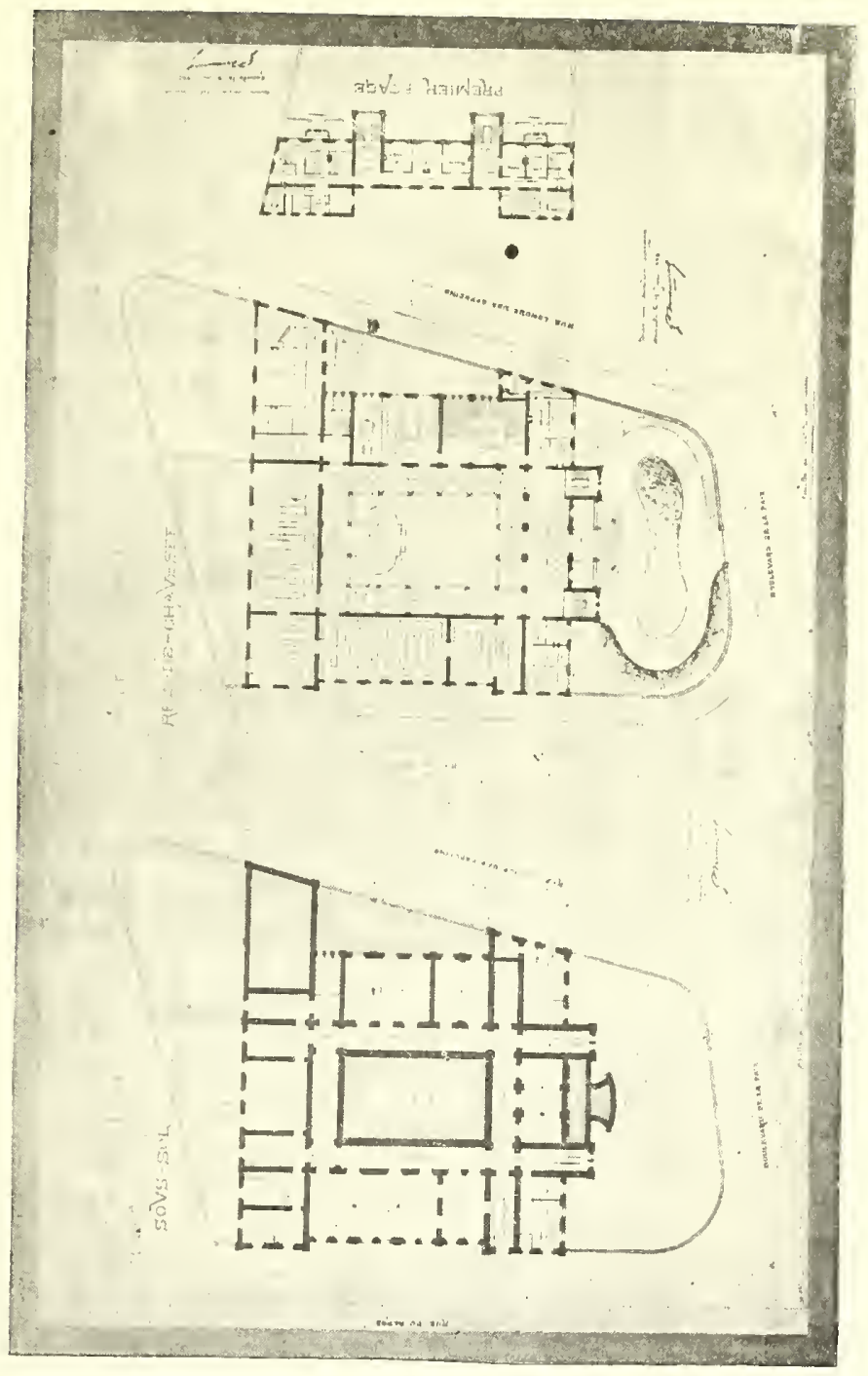

I'tan du rez-de-clinussie et des sous-sols de l'Jnstitut Colunial de Masscille projeté.

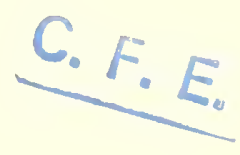




$$
-34-
$$

et peu étudiés de Madagascar, du Soudan et du Congo, immenses possessions récemment acquises. A cûté de ces salles, seront installés des laboratoires de recherches. Au rez-de-chaussée, on trouvera une vaste salle de cours et de conférences derant servir aux professeurs appelés chaque jour à donner l'enseignement colonial. On sait qu'il existe six chaires fondées par la chambre de commerce, relatives aux produits coloniaux te toute nature et à la science coloniale en général. D'autres cours seront institués par la chambre de commerce et il va de soi que les explorateurs et les conférenciers extraordinaires pourront aussi faire usage de cette salle et des collections de toute sorte du Musée.

Dans le sous-sol, il sera procédé au choix et au classement de tous les produits arrivés des colonies, et, au centre du rez-dechaussée, s'étendra un jardin colonial (serre chauffée en hiver). Il réunira les principaux types de plantes utiles provenant des colonies françaises et qui pourront. pendant les cours, ètre placées sous les yeux du public.

Au premièr étage, se trouveront une vaste salle de lecture attenant à la bibliothèque et à la salle des cartes coloniales; enfin le logement du directeur.

Tout cet ensemble constituera un monument à façade sévère mais ornementée, d'un développement suffisant en longueur et en largeur pour faire face à toutes les exigences du but à atteindre. Les dispositions intérieures indiquées sur les plans du monument joints à cette notice pourront être modifiées selon ces exigences; aussi ne jugeons-nous pas nécessaire d'entrer dans les détails de ce dispositif qui pourra subir au dernier moment des remaniements sils sont nécessaires.

Ainsi que nous l'avons dit, les plans ont été dressés par M. Mouriès. Ces plans, dans lesquels l'honorable architecte avait visé surtout à la commodité, à l'aménagement intérieur, n'ont pas reçu, d'abord, l'approbation du comité des bâtiments civils. Celui-ci a estimé que l'architecture des façades était formée d'éléments coloniaux qui donnaient à l'édifice une allure de fantaisie incompatible avec le 
caractère que ces façades devraient avoir pour contribuer à l'embellissement de la ville de Marseille et que, dans ces conditions, l'architecture extérieure devait être complètement changée. Le style primitif était celui qui a été adopté pour l'École coloniale de Paris.

M. Gustave Mouriès a donc été amené à tenir compte des modifications qui lui étaient signalées et à remanier son projet. C'est ce qu'il a fait. Le style qu'il a adopté se rapppoche, pour l'extérieur, du style Renaissance simple, et, pour ì grande courintérieure, avec promenoirs, du style mauresque. C'est une conception architectonique dans laquelle s'allient le goût à la simplicité, l'élégance à l’originalité et qui éveille bien l'idée de la destination et de l'emploi que doit recevoir cet édifice.

D’autre part, la ville a voté dàns la même séance du 28 juin 1898 , une somme de 26.300 francs pour la construction d'une vaste serre de plantes économiques tropicales qui sera édifiée au parc Borély dans la section coloniale du Jardin botanique. C'est là que pourront être entreprises plus à l'aise qu'elles ne l'ont été jusqu'ici dans les petites serres actuelles, des recherches s. r les plantes coloniales de grande ou de petite culture à amúliorer o à faire connaître. C'est, cependant, de ces modestes serres que so it sorties tous les plants d'Lraucaria Bidwilli qui ont été introduits dans nos colonies d'Afrique ou d'Océanie, sur les recommandations et après les travaux de M. Heckel sur cette espice dont la propagation doit être tentée partout où elle est possible, à cause des produits alimentaíres (graines) et industriels (gomme-résine) d premier ord re qu'elle donne assez abondamment. C'est aussi de ce laboratoire des serres que sont sortis tous les pieds de kola qui on: été introduits après les travaux de $\mathrm{M}$. Heckel, dans toutes nos ccl onics françaises tropicales (Antilles, Guyane, Madagascar, Indo-Chine, La Réunion) qui en avaientété dépourvues jusqu'alors”(1): le passé garantit donc ce qui se fera dans l'avenir, gràce à cet outillage nouveau.

(I) Nous donnons à lafin de cette notice avec quelques détails la composition du musée de Marseille et la nomenclaiure complète des produits exposés par le musée au Trocadéro. 
Quant aux plantes nouvelles dont la culture a été introduite dans nos colonies françaises par suite d'envois de graines faits par les semis de la section coloniale du Jardin botanique de Marseille, elles sont nombreuses et je dois me borner à citer, en raison de l'utilité ou de l'importance qu'elles ont prise dans le commerce ou dans l'industrie de la métropole: $1^{\circ}$ Le Kinkélibah remède africain végétal, en honneur contre la fièvre bilieuse hématurique et introduit partout actuellement; $2^{\circ}$ le Maloukang ou Ankalakı, graine africaine donnant un beurre comestible excellent et très agréable quand il est frais. Cette plante réussit bien en Indo-Chine et y donne de belles espérances entre les mains de $\mathrm{M}$. Achard, Inspecteur de l'Agriculture qui, comprenant tout le haut intérêt d'avenir pour sa colonie que présente cette plante économique, s'en occupe très activement. Il n'est pas douteux que ce sera, pour l'industrie des corps gras comestibles, une conquête de premier ordre, quand la plante sera acclimatée dans nos diverses colonies tropicales.

Nous allons passer maintenant en revue les travaux les plus importants et les plus récents qui sont sortis, ou vont sortir par la publication, des laboratoires de l'Institut colonial et nous insisterons particulièrement, à raison de leur portée économique, sur les résultats des recherches nouvelles de M. Jumelle, sur les caoutchoucs et les cacaos; de M. J. de Cordemoy sur les gommes et les résines; de $M$. Laurent surle tabac, enfin de $M$. Heckel sur les graines grassas nouvelles ou peu connues des colonies françaises. Nous indiquerons ensuite, ou chemin faisant, les travaux en cours qui vont bientôt paraitre. Les divers produits nouveaux avec leurs dérivés qui font l'objet de ces études, sont ceux qui forment le fond le plus important de l'Exposition actuelle du Musće colonial de Marseille au pavillon du Ministère des colonies, au Trocadéro. 


\section{RÉSUMÉ}

Des Travaux Botaniques les plus récents

RELATIFS AUX PLANTES COLONIALES ÉCONOMIQUES

Faits par MM. les Professeurs

Edouard HECKEL, JUMELLE, J. de CORDEMOY \& LAURENT

AU LABORATOIRE DE L'INSTITUT COLONIAL DE MARSEILLE

Sous la direction du Professeur Dr E. HECKEL

I. Recherche sur les plantes a caoutchouc des Colonies françaises par M. JUMELLE.

II. La culture et l'exploitation du cacaoyer dans tous les pays de production par M. Jumelle.

III. Un livre. de M. Jumelle sur les Cultures Coloniales s'imprime en ce moment chez M. J.-B. Baillière, éditeur à Paris.

IV. Recherches sur les gommes, résmes et gommes résines par M. J. de Cordemoy.

V. Recherches sur les graines grasses nouvelles ou peu connues des Colonies françaises par le professeur E. Heckel.

VI. Sur le tabac dans nos Colonies françaises par M. Laurent 



\section{I - LES CAOUTCHOUCS DES COLONIES FRANÇAISES}

ET NOIAMMENT CEUX QUI SONT EXPOSËS PAR LT MUSÉ COLONIAL

DE MARSEILLE APRÈS ÉTUDE

La question des plantes à caoutchouc, de leur culture et de leur exploitation est. à l'heure actuelle et de tous còtés, une de celles qui préoccupent le plus nos colons, en raison de l'importance, sans cesse grandissante du produit.

Depuis un certain nombre d'années déjà, M. .Iumelle s'est adonné tout spécialement à cette étude au Musée de Marseille, et, en 1898 , il publiait, dans les Amales de l'Institut, un travail où étaient réunis tous les documents, jusqu alors épars, qui concernaient nos colonies et où étaient résumés, en même temps, les résultats de recherches personnelles.

C'est ainsi qu'on trouve dans cet ouvrage ( Les plantes à caoutchouc et à gutta dans les colonies fiançaises) beaucoup de données nouvelles sur les caoutchoucs du Fernan-Vaz qu'expose le Musée et dont la plupart sont dus aux envois de M. Foret, alors administrateur du Congo français.

Les principales plantes productrices sont, en dialecte n'coumi: l'okouendé n'gow'a, le n'djembo, le gnongo, l'inogué, l'ébourendé, le loni, l'itomba, le bouéla, l'ogoumou, le gambo n'sina, et l'iganda. Toutes n'ont pas la même valeur. Les meilleures sont : le n'djembo, l'okouendi n'goura, le gnongo, l'ébourendé, le bouéla.

Pour quelques-unes, les échantillons botaniques ne sont malheureusement pas assez complets pour en permettre une détermination précise. M. Jumelle a pu toutefois ètudier assez complètement le n'djembo et l'okouendé n'gou'a. 
Le nidjembo reçu au Musée Colonial de Marseille (et il mporte de préciser, car le mème nom peut s’appliquer à d'autres lianes) est le Landolphia Foreti Jum, qui est sans doute la mêmı plante que celle que M. Pierre nommait, en mème temps, Lando!pliza Klainei. Son produit est aujourd'hui bien reconnu comme étant de valeur réelle, car des graines de la liane sont, depuis ces études, livrées au commerce par le Jardin botanique de Bruxelles et par le très habile horticulteur colonial français M. Godefroy-Lebeuf. 'Le caouchouc exposé ne contient, d’après les analyses faites au Musée, que 6 à 7 o/o de résine; il est très élastique. très nerveux et peu risqueux.

Des graines de Landolphia Foretiont germé au Jardin botanique de Marseille, où l’on conserve ainsi des spécimens vivants de l'espèce.

L'Okonendé n'gowa est le Carpodinus Foretiana Pierre. Sa gomme est plus résineuse que la précédente ( 19 o/o environ), elle n'en est pas moins une sorte encore au-dessus de la moyenne, comme valeur, et très utilisable, bien qu'elles’altère plus rapidement que le produit du n'djembo ou celui du gnongo.

Ce dernier est, avec le caoutchouc d'ebourendé, un des meilleurs à signaler, en même temps que les deux précédents.

Au contraire l'inogué (Carpodinus Jumellei Pierre)'donne une sorte inférieure. Il en est de mème du loni (55 o o de résine), du gombo n'sina $(54 \mathrm{o} / \mathrm{o})$, de logoumou $(50 \mathrm{o} / \mathrm{o})$.

M. Jumelle n’avait pu, d’ailleurs, dans l'ouvrage cité plus haut, donner pour toutes nos colonies des détails aussi nombreux quepour le Gabon-Congo, et il avait dû aussi laisser çà et là en suspens beaucoup de questions non encore élucidées.

Mais, depuis lors, le Musée de Marseille, dont les relations avec nos possessions extérieures s'étaient rapidement étendues, a reçu beaucoup d'échantillons qui lui ont permis d'éclaircir quelques-uns de ces points obscurs.

Les avis étaient, par exemple, très divisés sur la valeur réelle du caoutchouc du Landolphia florida, si répandu en Afrique, aussi bien sur la côte orientale quesur la côte occidentale. D’après Ficalho, le $\mathrm{Dr}$ Nourry et M. Lecomte, ce caoutchouc était sans valeur ; il était 
bon, au contraire, d'après Speeke et Grant, et MM. Moreller et Willey Après l'examen fait, au musée, despécimens de la lianeet du produit, il ne peut rester aucun doutz: c'est à l'opinion de Ficalho et du Dr Nourry qu'il faut se rallier.

M. Adam, administrateur de Ia Casamance, nous envoyait, en effet, il y a deux ans, des frag nənts d'une plante appelée mada au Sénégal. M. Jumelle y a reconnu le Landolphia florida. Or le produit qui accompagnait les échantillons botaniques est tellement inférieur qu'on n'a pu avoir un seul instant, l'intention de l'exposer.

D'autre part, c'est grâce à un autre envoi de M. Adam que M. Jumelle a pu établir - cequi a été confirmé depuis par d'autres observations de $\mathrm{M}$. Hua - que la principale liane à caoutchouc toll du Sénégal est, non pas le Landolphia senegalensis. comme on l'admettait. jusqu alors, mais le Landolphia tomentosa. Ajoutons que d'après M. Hua, ce serait ce mème Landolphia quisaraitlegohine du Soudan.

Nos connaissances sur les végítaux caoutchoutifères de nos colonies de la côte occidentale d'Afrique se précisent donc ainsi peu à peu. Mais le Musée de Marseille a eu, en outre, la bonne fortune de trouver, à Madagascar, en M. Perrier de la Bathie (qui réside à Suberbieville), un correspondant obligeant et zélé. dont les nombreux envois, faits avec grand soin, ont fourni à M. Jumelle une abondante matière à d'importantes observations.

Il faut bien avouer aussi que, pour notre nouvelle colonie africaine, toute l'histoire des plantes à caoutchouc était encore à faire. Il y a deux ans, tout était dit, au point de vue scientifique, quand on avait rappelé que le caoutchouc de Madagascar est fourni par le Landolphia madagascariensis. On savait seulement encore que dans le Sud était une euphorbiacée, 'l 'intisy".

Qu'on était loin, pourtant, de la réalité! Peut-être mème le Landolphia madagascariensis (quiest le yahena de la côteorientale) n'existe-t-il pas dans la région du Nord-Ouest de l'île, qui, cependant exporte de grandes quantités de gomme.

C'est du moins, ce qui semble ressortir, jusqu'ici, de l'examen de tous les échantillons envoyés au Musée par M. de la Bathie, et qui 
représentent à peu près toutes les plantes à caoutchouc exploitées ou exploitables dans le Bouéni et dans le Ménabé.

Et ce seraient deux autres espèces du même genre qui remplaceraient, dans cette partie de l'ile, le Landolphia madagascariensis.

De ces deux espàces, qui n'avaient pas été encore décrites, l'une a été appelée par M. Jumelle le Landolphia Perrieri. C'est le piralahy ou vahealahy des Sakalaves, qui, pour en récolter le lait coupent ses rameaux par tronçons. Les fragments de la liane sont ensuite égouttés au-dessus d'un bambou creux qui conduit le lait dans un récipient. La coagulation est obtenue par le jus de citron ou avec des fruits pilés de tamarinier, car les indigènes ont bien su reconnaitre d'eux-mêmes que l'ébullition ne donne qu'un produit inférieur.

La liane pousse dans toutes les forêts du Bouéni, à Majunga, à Andriba, dans les vallées de l'Ikopa, du Betsiboka et du Menavava. Le caoutchouc reçu et examiné au Musée est de bonne qualité, très élastique, et sans viscosité. Il contient 8 à 9 o o seulement de substances étrangères (parmi lesquelles la liqueur de Fehling ne décèle pas la moindre trace de sucre) et 4 à 50 o de résine. Sa densité est remarquablement faible : 0,910, alors que celle du caoutchouc de Para qui est généralement citée comme une des moins élevées, est de o,915 et celle du caoutchouc d'Assam (Ficus elastica) 0,967 .

Le latex, qui est de conservation très facile quand on l'additionne d'un peu d'ammoniaque, et qui est arrivé au musée en très bon état, est blanc légèrement rosé et très fluide. Sa densité est 0,997. Il est malheureusement très pauvre en caoutchouc. Le rendement de l'échantillon que nous avons examiné n'était que de 6 à $7 \%$, alors que celui de la plupart des autres laits est de 20 à 50 . Il est vrai que, à la fin de la saison sèche, il est plus élevé, mais, mème à cette époque il n'atteintque i 7 o 0 .

Cette extraordinaire pauvreté est d'autant plus regrettable que la gomme est bonne et que la liane, mème après avoir ćté coupée au ras du sol, repousse facilement, donnant un grand nombre de rameaux qui s'enchevêtrent en buisson. Il serait peut-être ainsi possible, d'après M. Perrier de la Bathie, d'exploiter la plante, par coupes régulières, tous les deux ou trois ans. 
Ce sont des essais qu'il serait à propos de tenter, car, actuellement l'exploitation du piralahy est presque abandonnée par les Sakalaves dans le Bouéni. Et le faible rendement explique cet abandon, car un ouvrier peut difficilement récolter, pendant la saison des pluies, plus de huit litres de lait par jour. Or ces huit litres ne donnent qu'un demi kilo de caoutchouc, vendu sur place au faible prix de 1 fr. 50 ou 2 francs le kilo.

La seconde espèce de Landolphia a laquelle il est fait allusion plus haut est particulièrement intéressante. Elle est bien connue des Sakalaves sous le nom de reiabo, et son caoutchouc, d'une belle couleur rose, doit constituer certainement, en grande partie dans la région, la sorte très estimée connue sous le nom de pinki rose de Madagascar.

Il n'en était que plus important, au point de vue scientifique, de déterminer botaniquement la plante productrice que, d'après certains voyageurs, les Sakalaves ne faisaient pas connaitre volontiers. C'est, sans le moindre doute, d'après les échantillons que possède le Musée, une espèce non décrite encore, et bien distincte. par ses fruits, du Landolphia madagascariens is et du Landolphia Perrieri. Ces fruits, qui sont pyriformes dans la première de ces deux espèces et qui, ovoïdes et surmontés d'un mamelon, ont un peu la forme d'un citron dans la seconde, sont sensiblement sphériques dans le reiabo et justifient ainsi le nom de Landolphia spharocarpa quion peut donner à l'espèce. Ils contiennent plus d'une centaine de graines, noyées dans la pulpe sucrée. Leur dimension la plus ordinaire est celle d'une grosse pomme: elle peut ètre cependant plus grande encore car un des spécimens exposés, qui pesait, à l'état frais, 480 grammes, a Io centimètres de diamètre.

Une de leurs principales caractéristiques est la grande quantité de lait que contient l'enveloppe et qui est telle que lorsqu'on brise cette enveloppe sèche, on voit s'étirer, entre les fragments, de nombreux fils de caoutchouc. Un fait analogue ne se produit pas nécessalrement pour les fruits d'autres espèces de Landolphia. Le fruit du reiabo est encore très souveiıt, en raison de cette même abondance de lait, parsemé, à sa surface de petites parcelles de caoutchouc. Ces 
parcelles proviennent évidemment de la coagulation de quelques gouttes de liquide qui ont jailli à la suite d'une piqûre d'insecte.

Des recherches plus précises sur la gomme sont, en ce moment en voie d'exécution au Mlusée, mais un examen même rapide suffit pour convaincre immédiatement de sa valeur.

Le tronc de la liane est plus gros que celui du piralahy et il doune un lait plus abondant et plus riche en caoutchouc ( 18 à 26 o/o). Le mode d'exploitation est le même.

Le Landolphia spharocarpa serait spécial au Ménavava et au Ménabé; on ne le trouve plus à l'est d'une ligne indiquée par la vailée du Ménavava et celle du Betsiboka, après le confluent avec cette rivière. Partout en cette région il pousse avec une vigueur très grande dans les alluvions qui bordent les cours d'eau et qui sont les seuls terrains cultivables du pays.

La liane pourrait ètre cultivée, d'après $M$. Perrier, avec quelques chances de succès; et, contrairement au piralahy, elle pourrait être saignée.

Voilà donc, dans le nord-est de Madagascar, deux Landolphia producteurs de caoutchouc, et autres que le Landolphia madagascariensis.

Mais il ressort nettement, en outre de l'étude des plantes adressées au Musée, que le genre Landslphia n'est pas le seul, parmi les Apocynées, qui fournisse dans l'ìle des végétaux caoutchoutifères. Et au second rang il faut tout au moins placer un autre genre de la même famille, sur lequel, avant cette année, l'attention n'avait jamais été attirée à ce point de vue : c'est le genre Mascarenhasia.

C'est M. K. Schumann, de Berlin, qui, au commencement de 1898 , reconnut que l'arbre mgoa, qui peut fournir, sur la côte d'Atrique dans la région de Zanzibar, une gomme élastique, est un Mascarenhasia qu'il nomma M. elastica. L'importance du genre, au point de vue où nous nous plaçons ici, n'était toutefois pas, par là établie, car il pouvait s'agir d'une espèce isolée. Il en est tout autrement depuis que les recherches de $M$. Jumelle ont établi que les arbres exploités par les Sakalaves sous le nom de guidroa sont des congénères du mgoa de Zanzibar. 
Le principal de ces guidroa du Bouéni et du Ménabé est le Mascarenhasia lisianthiflora D. C., petit arbre de 5 à 6 mètres de hauteur. Les Sakalaves, pour l'exploiter, pratiquent sur le tronc, pendant la saison sèche alors que le lait est très épais, de nombreuses incisions. Le lait se coagule presque immédiatement, au-dessous de la blessure, en formant de petites bandes de gomme, que les travailleurs reviennent enlever une heure plus tard, et qu'ils agglomèrent en boule. Un seul homme, par ce procédé, récolte facilement un kilo de produit en une journée. Un litre de lait donne en moyenne 41 5 grammes de caoutchouc de bonne qualité, mais bien distinct de celui du reiabo par sa couleur noir foncé.

Un autre guidroa de la même contrée est le Mascarenhasia anceps Borv., qui pousse sur le bord des ruisseaux et des étangs, et qui est commun dans tout le Bouéni, à Majunga, à Suberbieville, à Menavava, etc.

Le caoutchouc en est encore bon. Malheureusement, en raison de l'habitat plus ou moins aquatique du végétal qui le fournit, le latex est très clair et ne donne que peu de gomme. La plante est, pour cette raison, délaissée des Salialaves. D’apris une observation curieuse de $M$. de la Bathie, les saignées pratiquées aux premières pluies donnent cependant une assez grande quantité de liquide pour que l'exploitation, à ce moment, en raison de la grande fréquence de l'arbre, soit rémunératrice.

Et on pourrait, de même, tirer parti d'un troisième Mascarenhasia à teuilles étroites, en ce moment à l'étude au Musée et qui fournit un produit valant celui du $M$. lisianthiflora. Celui-là est un arbre de 20 à 30 mètres de hauteur, d’où le lait coule en très grande quantité. Il est regrettable qu'il soit rare dans la région. M. Perrier de la Bathie n'en a trouvé que quelques pieds dans une forèt à demi inondée, près du lac d'Hoyefitra, aux environs du mont Isibondraina.

Peut-être, au reste, est-ce une espèce qu'on retrouve plus communesur d'autres points de Madagascar, car le genre Mascarenhasia n'est certainement pas limité au Nord-Ouest. Si on se reporte aux descriptions - si incomplètes qu'elles soient -- données, dans 
diverses publications, par des colons ou des explorateurs, il apparaît certain qu'il a aussi des représentants dans le Nord et dans l'Est. L'haqondrano, grand arbre de la région de Vatomandry et Farafangana. l'herotra de la zone forestière comprise entre Mananjary et Fianarantsoa, le barabanja, du Nord, sont des Marcarenhasia.

Mais revenons à la partie occidentale. Après les Apocynées précédentes deux Asclépiadées doivent encore être signalées au même titre.

L'une est le lombiro des Sakalaves, bien sourent cité, mais qui n’avait jamais été déterminé: c'est le Cryptostegia madagascariensis. Et il était intéressant de connaître de façon précise le produit de cette plante, sur laquelle aucune donnée n'avait été encore fournie, alors qu'on possède, au contraire, quelques renseignements sur la seconde espèce dugenre: le Cryptostegia grandiflora

L'origine de cet autre Cryptostegia, à vrai dire est douteuse. On a trouvé la plante, à l'état sauvage à la fois dans l'Inde et dans les îles africaines de l'Océan indien, sans qu'il ait été possible de reconnaitre dans laquelle de ces régions elle est réellement indigène. Mais il n'y a pas de doute, en tous cas, sur la valeur de la gomme. M. Deroux, dans une communication faite en 1887 à la Chambre d'agriculture de la Réunion, disait que des spécimens, envoyés en France avaient été très appréciés: et, d'autre part, dès 1856 , on pouvait voir, à l'Exposition de Madras, des échantillons du même caoutchouc apportés des listricts de Nellore, de Caddapah, de Mazulipatuam et de Madras et, qui. d’après les comptes-rendus, étaient de bonne qualité : c'est la gomme qui, dans l'lnde est connue sous le nom de Palay rubber.

Que fallait-ildonc penser de la valeur du produit du Cryptostegıa madagascariensis? On pouvait se le demander, et la réponse à cette question d'après les échantillons reçus par le Musée, ne sembla pas d'abord des plus faciles. Les premiers spécimens envoyés étaient, en effet, dépourvus de toute valeur: c'étaient des fragments d'une résine brunâtre, cassante, dépourvus de toute élasticité. Et cette observation concordait avec les lignes qu'écrivait (in litt.) M. Perrier de la Bathie : «Le latex de Lombiro ne m’a jamais donné qu'un pro- 
duit visqueux et inatilisable. L'acide sulfurique, l'acide citrinue, l'alcool donnent les mèmes résultats. »

Pourtant un peu plus tard, M. le Dr Decorse, médecin des colonies, adressait au Musée, en mème temps qu'une lettre dans laquelle il affirmait la bonne qualité de la gomme, un litre de lait.

Or M. Jumelle, en expérimentant sur ce lait, qui fut traité par divers coagulants, obtenait en effet un véritable caoutchouc.

Cette contradiction résultait-elle donc d'une erreur dans l'un des deux envois? La compétence incontestable des deux correspondants ne permettait pas de le supposer. Et l'explication - bien simple, mais bien curieuse, car c'est le premier exemple aussi net du fait que nous allons signaler, dans l'histoire des plantes à caoutchouc - se trouve dans une lettre récente de $\mathbf{M}$. de la Bathie : la composition de la gomme présente des variations considérables suivant l'époque de la récolte.

M. Perrier de la Bathie avait, en effet. lui aussi, à une époque postérieure à celle où il écrivait la phrase citée plus haut, obtenu un bon caoutchouc de lombiro... tandis que, en février dernier, il n'obtenait plus de nouveau qu'une substance résineuse.

La conclusion serait donc que le Cryptostegia madagascariensis donne du caoutchouc, de juin à décem bre, c'est-à-dire pendant la saison sèche (au cours de laquelle le Dr Decorse avait fait sesexpériences), mais ne donne qu'un produit résineux et sans élasticité pendant la saison pluvieuse. Des recherches plus complètes sur ce caoutchouc sont poursuivies en ce moment au Musée colonial de Marseille.

Enfin M. Jumelle a examiné aussi, dans ces derniers temps, la gomme d'une plante que M. Perrier lui a adressée sous le nom de bokalahy. Ce bokalahy, recueilli entre la rive gauche du Betsiboka et la rive droite du Mahavary, a un port tout semblable à celui dü lombiro. Abandonnée à elle-mème, la plante s'arrondit en buisson, alors que, au voisinage d'un arbre, elle devient une grande liane. C'est, d'après la détermination de M. Jumelle, le Marsdenia verrucosa, caractérisé. en partie, par son gros follicule verruqueux d'où s'échappent de nombreuses graines, surmontées de volumi- 
neuses aigrettes. (1) C'est encore une espèce qui n'avait jamais été, d'ailleurs, signalée comme caoutchoutifère. Sur le genre même, pourtant sivaste, on ne possédaitjusqu'ici, à cepoint de vue, que cette ancienne et vague indication de Roxburgh, que, dans l'Inde « le lait qui sort des incisions du tronc du Marsdenia tenacissima donne une substance élastique, qui ressemble à du caoutchouc. »

Il convient, il est vrai d'ajouter, que le Marsdenia verrucosa ne parait pas appelé à prendre une bien grande importance pratique. Le latex est, d'abord, en trop petite quantité pour qn'on puisse l'exploiter avec profit pour le moment; et le caoutchouc, tout en étant de bonne apparence, élastique et peu risqueux (16.5 o o de résine) est assez peu tenace. Il casse facilement lorsqu'on l'étire tel qu'on l'obtient après coagulation; et il est encore moins tenace lorsqu'il a été débarrassé de sa résine.

Quoi qu'il en soit, on voit maintenant comment, en ces derniers temps, les nombreux spécimens envoyés par d'obligeants correspondants au Musée de Marseille nous ont permis d'étendre nos connaissances sur les plantes à caoutchouc exploitées ou exploitables dans nos colonies africaines.

Il serait à souhaiter qu'on pût prochainement en dire autant de notre colonie de l'Indo-Chine, où la récolte du caoutchouc pourrait être aussi une source de revenus peut-être un peu trop négligée jusqu'ici. Les végétaux producteurs de cette contrée sont malheureusement encore mal connus et indéterminés, et leur étude nous réserve pourtant certainement des surprises. Ainsi, tout dernièrement, M. Achard, directeur du service de l'agriculture en Indo-Chine envoyait au Musée, en priant de l'examiner au point de vue botanique, une liane qui serait parait-il, caoutchoutifère. M. Jumelle y a reconnu le Vallaris Heynei Spreng. (Vallaris solanacea Sch.) qu'on n’a jamais signalé comme espèce à gomme élastique, bien qu'elle soit commune, croyons-nous, dans l'Inde. C'est ainsi une nouvelle plante à ajouter à la liste précédente. Les caoutchoucs qui font I'objet ủe l'examen ci-dessus sont exposés au Trocadéro par le Musée colonial de Marseille.

(1) On peut vorr au jardin bortanique de Marseille des spécimens vivants de ce Marsdenia perrucosa, ainsi que du Landolphıa spharocarpa. Les uns et les autres proviennent des semis faits en serre. 


\section{1i. GOMMES ET RESINES DES COLONIES FRANCAISES}

PAR M. J. DE CoRdemoy

Le travail que M. le Dr Jacob de Cordemoy a publié sous ce títre cette année dans les Annales de l'Inslitut Colonial de Marseille, sur les Gommes, Résines et Gommes-résines d'origine exotique a un double but: $1^{0}$ rassembler des documents épars, réunir dans un cadre méthodiquementordonné des notions et des faits qu'il y avait intérêt à étudier comparativement; $2^{0}$ faire connaître des produits nouveaux ou peu connus que nos colonies pourraient fournir au Commerce et à l'Industrie, qui, par suite, deviendraient pour quelques-unes de nos possessions d'importantes sources de revenus.

En faisant l'histoire des Gommes, l'auteur a longuement parlé de la gomme du Sénégal et du Soudan dont la production constitue une véritable richesse pour ces colonies, et a ånalysé avec soin les conditions de récolte et d'exportation de ce produit qui a pour marché Bordeaux et est presque entièrement consommé par l'industrie française. Mais il a montré que les importations. en France, des gommes exotiques comportaient en outre nombre de sortes étrangères dont les similaires se trouveraient aisément dans nos propres colonies. Il a cité, par exemple, l'Acacia Farnesiana, qui croît si bien aux Antilles et à la Réunion. comme susceptible de fournir une excellente gomme, tout à fait analogue à la gomme arabique; le Feronia elephantum de nos possessions de l'Inde, donne également une magnifique gomme, de qualité tout à fait supérieure. La gomme de l'A cacia lebbek si répandue dans nos colonies, est, il est vrai, insoluble, mais l'auteur a indiqué des moyens de la rendre soluble.

Parmi les gommes du type adragante, M. de Cordemoy a tout spécialement signalé le M'Beppe ou gomme du Sterculia tomentosa, sur laquelle $\mathrm{M}$. le professeur Heckel avait, quelque temps auparavant, attiré l'attention. C'est un produit que la Sénégambie française pourrait livrer à l'industrie française qui la transformera facilement en gomme soluble.

Le groupe des résines offre tout une série de matières premières de réelle valeur, susceptibles de devenir l'objet d'un commerce 
lucratif, si elles pouvaient donner lieu à des transactions suivies. L'étude des gommes et des résines d'Araucarias. du Copal de Madagascar est fort instructive à cet égard; il en est de mème des indications fournies au sujet des Damars et de la résine des Gardenia de la Nouvelle-Calédonie. Ces substances forment dexcellents vernis capables de répondre à toutes les exigences de notre industrie qui utilise seulement jusqu'ici le copal et la résine damar de provenance malaise.

Un chapitre assez étendu est consacré à la gomme laque asiatique que le Laos et le Cambodge produisent abondamment, et qui donne lieu à des transactions importantes sur les marchés de l'IndoChine.

De la gomme laque d'Ásie, l'auteur a rapproché un produit ana. logue, provenant de Madagascar : c'est une résine laque blanche dont les caractères sont bien distincts, mais dont les propriétés sont telles qu'elle semble se prêter aux mêmes usages que la 'gomme laque carminée.

L'auteur passe en rerue les différentes sortes de résines qui découlent des nombreuses espèces de la famille des Diptérocarpées. Il s'est efforcé de mettre en lumière leu r origine botanique précise de manière à montrer quels sont ceux de ces végétaux qui méritent plus spécialement d'être protégés et propagés. Tels sont en Indo-Chine les Shorea qui fournissent la résine Chaï et les Dipterocarpus qui donnent l'huile de bois.

Il a mis au point de nos connaissances actuelles la question complexe des produits résineux secrétés par les Burséracées et à pu notamment en mettant à profit les collections du Musée-Colonial de Marseille - fixer, après des recherches personnelles, quelques-unes des propriétés les plus remarquables des résines de Canarium, de Protium, de Bursera et de Dacryodes.

11 a élucidé également quelques points douteux concernant les produits résineux des $S_{y}$ mphonia et des Moronobea. Ces investigations laissent supposer que le Kijy ou résine de Symphonia clusioides, dont on peut se procurer de grandes quantités à Madagascar, donnerait un vernis utilisable industriellement.

Les renseignements qu'il a été possible de recueillir sur le ben- 
join seront sans doute de quelqu'utilité dans notre colonie de l'lndoChine où l'arbre à benjoin est relativement peu commun et mériterait d'être plus cultivé et propagé.

Il y a également en lndo-Chine une laque noire, gomme-résine tout à fait analogue à la laque bien connue du Japon et de la Chine; elle est fournie par le Melanorrhea laccifera, et donne, mélangée à l'huile de Trau (Aleurites cordata), un magnifique vernis très employé par les Cambodgiens et les Annamites.

Enfin, l'auteur a rappelé, en s’inspirant des remarquables recherches publiées par le Dr Heckel, la grande importance économique des diverses espèces d'Araucaria, comme végétaux producteurs de gommes et de résines.

Tels sont les principaux chapitres de ce travail; un très grand nombre d'autres plantes gommifères et résinifères ont été étudiées, en notant pour chacune d'elles la valeur économique véritable de l'exsudat.

Ce premier volume sera bientòt suivi d'un second, actuellement en préparation, où sera faite l'histoire des Plantes féculentes et des fécules dans nos colonies françaises. On trouve là des cultures de tout premier ordre. comme le riz, le principal produit dexportation pour notre colonie de l'Indo-Chine, le maïs. le manioc, le mil, l'arrowroot, etc, dont certaines possessions pourraient fournir des quantités considérables.

M. de Cordemoy a commencé aussi à réunir des matériaux pour une étude sur les Epices et les aromates dont nos colonies nous offrent une collection aussi riche que variée. Il developpera surtout la partie qui, dans ce travail, sera réservée à la vanille; la culture de cette Orchidée est. comme on le sait, très en honneur dans l'une de nos vieilles colonies, la Rźunion, qui trouve dans le commerce des précieuses « gousses » le plus clair de ses revenus. Il est probable quà Madagascar nos colons auront intérêt à être documentés sur la culture et l’industrie de la vanille, car la plante y pousse à merveille, dans certaines régions du moins.

Le même auteur a l'intention d'écrire également un volume sur la Canne à sucre. Il y a lieu, en effet, de faire une révision des 
anciens traités publiés sur cettequestion, ne serait-ce que pour y ajouter les récentes recherches sur la sélection de la canne par la méthode des semis, sur certaines de ses maladies, et le moyen de les prévenir ou de les combattre, etc.

En dernier lieu, il soccupera d'une série de monographies concernant nos Fruits tropicaux, dont beaucoup seraient certainement très appréciés en Europe si l'on parvenait à découvrir un procédé permettant de les conserver à l'état frais pendant le transport. Quelques essais ont été tentés, en ce qui concerne les Antilles; mais la question ne paraît pas résolue complètement. Toutefois on constate avec satisfaction au Pavillon de la Martinique, dans la salle de dégustation, l'existence d'un rayon spécialement réservé aux fruits fraís. On peut se procurer là, à assez bon compte, lc plaisir de déguster des pommes lianes, des arocats, des pommes cannelles, des pommes Cythères, etc. etc. surtout d'excellentes mangues. C'est aux efforts de M. Landes, professeur au lycée deSaint-Pierre, agent de la colonie à la section des Antilles, que ce magnifique résultat est dû et il faut l'en féliciter hautement. C'est le premier pas vers l'exportation normale des fruits frais des Antilles. Le Sénégal étant plus rapproché de nous, la réalisation de ce progrès pourra nous être mieux assurée par cette colonie, quand elle sera en état d'exporter. 


\section{III. - GRAINES GRASSES NOUVELLES OU PEU CONNUES}

Notamane Gelles QUi FIgURENT A L'expositiox DU Trocadero

ET PROVENANT DES COLONIES FRANGAIsEs, PAR ED. HECKEL.

On semble admettre dans certains milieux industriels que les graines grasses coloniales connues et exploitées sur une grande échelle en ce moment pour la fabrication du savon et des bougies, voire mème pour l'alimentation, se réduisent à un petit nombre d'espèces, telles que l'arachide, le sésame, le ricin, le pourghère. pour les huiles liquides, et les coprahs. les illipés, pour les huiles concrètes; on ne croit pas devoir chercher au-delà parce qu'on ne pense pas pouvoir trouver mieux. C'est là une erreur préjudiciable au développement de l'industrie des corps gras, si prépondérante à Marseille,et c'est pour la dissiper et orienter les efforts des industriels vers un courant d'approvisionnement plus fructueux, que M. Heckel a entrepris une série de recherches sur nos graines grasses coloniales les plus importantes et les moins connues encore et même sur quelques-unes qui sont jusqu'ici absolument inconnues. Publiées dans les Annales de l'Institut colonial de $189+$ à 1899 , ces recherches méthodiques et aussi complètes que possible (elles englobent l'étude du végétal producteur et de la graine à tous les points de vue) ont eu d'heureux résultats comme il fallait s'y attendre, car plusieurs graines abondantes dans nos possessions de l'Afrique occidentale (Guinée et Congo), telles que le lamy. le bouandjo. le méné, le carapa, l'ourala, le karité, le maloukang d'Afrique et le krébao de l'lndoChine (pour ne citer que les plus importantes), se sont montrées à la fois très riches en huiles concrètes et donnant des acides gras stéariques de haute valeur industrielle à tous les points de vue. Les tourteaux eux-mêmes de ces graines grasses (et cest un produit secondaire à ne pas dédaigner) se sont montrés quelquefois de bonne valeur nutritive pour les bestiaux ou d'excellents engrais.

La première partie du travail de $M$. Heckel a paru dans les Annales de l'Institut colonial de 1894 , la seconde, plus ample, en I 899 et la dernière paraitra en fin igoo, formant un ensemble richement pourvu de figures, de 300 pages environ. Quelques-uns des produits compris dans cette étude sont ou vont être englobés dans un courant commercial nouveau. Evidemment, il sera profitable i 
la colonie qui les possède sans en tirer jusqüici aucun parti, et à l'industrie marseillaise dont les rapports transactionnels avec la côte d'Afrique sont si bien assurés, à raison mème des besoins industriels spéciaux à cette grande cité manufacturière. Sans insister sur les graines huileuses qui n'offient qu un intérèt scientifique, pour le moment du moins, et qui,soit à cause de leur pauvreté en huile, soit à cause de leur rareté actuelle, soit à raison du manque d'application de leur produit à une industrie actuellement existante, soit pour toute autre raison, ne paraissent pas devoir sortir immédiatement de leur obscurité, conme. par exemple, le pongam de l'Inde, l'eloyqégué et le coula du Congo et du Gabon, l'ochoco et l'odjendjé de la même région, le kokun de l'Inde, le panda et l'enyessan du Congo, l'isano ou onguéko du Gabon, etc., etc., nous mettrons ici en lumière. la valeur de celles qui ont atteint ou sont appelées prochainement à atteindre un grand avenir industriel à raison de leur composition même, si l'intérêt des industriels et des négociants est attiré utilement sur leurvaleur, comme il faut le désirer :

10 Le lamy ou kanya (Pentadesma butyracea Don) est le plus intéressant de tous parce quill est produit par un arbre qui existe en forêts à côté du Méné et est commun dans notre Guinée, ensuite parce que le rendement de sa graine (toute décortiquée à l'état naturel) est de 33 o o, enfin parce que ce corps gras solide donne 81.65 d'acide gras solide de distillation (acide stéarique). Le tourteau est aussi un bon engrais; $2^{\circ}$ le karité (Butyrospermum Parkii Kotschy) est déjà entré dans l'industrie stéarique et y pénétrera de plus en plus, s'il peut arriver à la còte d'Afrique à l'état de graines d'un prixacceptable.C'est un bon produit à tous égards, qui vient du Soudan où l'arbre abonde en forêts. La graine donne 32 o/o d'un beurre solide employé par les indigènes comme aliment et qui renferme 43.5 o o d'acides gras solides de distillation (acide stéarique). Le tourteau, excellent au goût et riche en azote, est mèlé au cacao dans les chocolats de qualité inférieure (Espagne). Ce beurre est connu sous le nom de beurre de Galam, il est de grand avenir industriel; $3^{0}$ l'oy ala est une graine plate, brune et lisse vernissée, fournie par le Pentaclethra macrophylla Bentham, du Gabon et du Congo. Dans ce dernier pays on l'appelle paña. Le végétal (grand arbre) est 
commun surtout dans la forèt de Mayombé (Congo français). Le rendement de la graine, non décortiquée, en huile concrète (la décortication est très difficile) est de $36 \mathrm{o} / \mathrm{o}$, la distillation des acides gras donne 38 o o de belle stéarine qui fait de magnifiques bougies vernissées dont le point de fusion est très élevé $; 58.7$ degrés). Le tourteau amer est un excellent engrais contenant $30.5 \mathrm{o} / \mathrm{o}$ de matières albuminoïdes; 4" le maloukang ou ankalaki est une graine nourelle des contreforts du Foutah-Djallon, appelée, par M. Heckel, Polygala buty"racea et étudiée avec soin pour ètre propagée dans nos colonies chaudes à cause de sa valeur. Très petite, mais moins que le sésame, elle est fournie par une petite plante qui la porte dès la première année de semis. Le beurre qu'elle donne, quand elle est fraîche, rappelle le meilleur beurre de vache, mais son rendement est assez faible $(17.53 \mathrm{o} / \mathrm{o})$. Ce corps gras blanc jaunâtre donne $67 \mathrm{o} / \mathrm{O}$ d'acide gras de distillation formé d'un mélange de palmitine, de myristicine et d'acide palmitique libre. Ee corps gras entrera certainement dans l'alimentation soit en Europe, soit dans nos colonies; la culture, surtout en Indo-Chine (Java ayant donné l'exemple), commençant à s'occuper sérieusement de la plante. sous la conduite de M. Achard. Déjà cette graine pénètre en Hollande pour la fabrication des beurres artificiels (margarine); $5^{\circ}$ le bouandjo, fourni par l'Allanblackiaflortbunda Oliver. est une graine d'arenir en raison de sa grande valeur et parce que, vraisemblablement, l'arbre qui la fournit abondamment, réputé rare aujourd'hui, semble devoir se trouver avec abondance dans le haut Congo, l'Oubanghi et sur la route suivie par le colonel Marchand. Il en a été longtemps ainsi du lamy dont M. Famechon a trouvé récemment de véritables forêts à côté du méné dans la Guinée française. Pour le moment on ne connait le bouandjo que dans le Congo et le Gabon, mais rare. Ses graines, assez volumineuses, mais qui n'exigent aucune décortication préalable commele lamy, donnent $460 \%$ de corps gras solide, jaune foncé, renfermant 92.35 o/o d'acides gras solides de distillation (acide stéarique). C'est, indiscutablement, une des graines à rendement industriel le plus élevé en matière stéarique : en outre, la blancheur, le point de solidification $\left(62^{\circ} 3 \mathrm{e}\right)$ de cet acide la feront sùrement rechercher par 
l'industrie. Le tourteau fournit un bon aliment pour les bestiaux mais peu azoté; $6^{\circ}$ le krébao ou chung-bao de Cochinchine a déjà fait son apparition dans le commerce de Marseille et y restera probablement. La graisse qu'elle donne est demi solide; l'arbre qui fournit la semence est l'Hydnocarpusanthelmintica Pierre. de Cochinchine, où il est commun. L'amande, dépouillée de sa coque dure, donne un corps gras semi-solide blanc, rancissant rapidement. à odeur agréable d'axonge, et dont le rendement en acides gras solides de distillation (acide laurique) est de $73.80 \mathrm{o} / \mathrm{o}$. Leur point de solidification est de $37^{\circ} 9$. Ce corps gras ne saurait avoir un emploi en stéarinerie à cause du faible point de solidification des acides gras solides, mais il pourrait ètre d'un grand secours pour les savonniers. Le tourteau, assez riche en matières albuminoïdes, constitue un bon aliment pour les bestiaux; $7^{\circ}$ les carapas: il en existe deux, celui dit touloucouna, propre à l'Afrique chaude, et celui dit carapa, propre à la Guyane. Mais ces deux graines sont produites par deux espèces voisines du même genre Carapa, savoir : C. Guianensis Aublet et C. Touloucouna Guill et Perr. La première graine est la plus intéressante parce qu'elle abonde dans les forêts de la Guyane où elle attend une exploitation. La graine décortiquée donne $30 \mathrm{o} / \mathrm{o}$ d'une huile liquide qui rend 49.28 o o dacide gras de distillation (acide palmitique) dont le point de solidification est de $39^{\circ} 20$. Cette huile a son emploi marqué dans l'industrie stéarique. Les localités les plus importantes de notre Guyane où ce végétal existe en massifs forestiers importants sont: le Cachipour. le Carséwène, le Couanany et surtout l'Oyapock. Il conviendrait d'établir une huilerie sur place dans les forêts. Quant au touloucouna du Sénégal et surtout du Haut-Niger, il donne une graisse solide mais de moindre importance. quoiqu'elle soit plus abondante dans la graine $(43.640 \%)$, et elle pourrait ètre utilisée que dans la savonnerie. Le rendement en acides gras de distillation est de $830 \%$ o, celui en acides gras solides de distillation de $48 \mathrm{o} / \mathrm{o} \mathrm{et}$ le degré de solidification des acides gras de distillation est de $39^{\circ}$. Comme dans l'huile de Carapa, on trouve dans le beurre de touloucouna de la palmitine et de l'oléine, mais ces glycérides existent en proportions inégales dans les deux graisses. Les tourteaux de Carapa 
d'Afrique ou d'Amérique, inutilisables en tant qu'aliments à cause de leur amertume, sont de très médiocres engrais à cause de leur faible ieneur en azote et en acide phosphorique: $8^{\circ}$ enfin le méné clôt actuellement la liste de nos graines nouvelles des colonies françaises. Elle est fournie par le Lophira alata Banks et enfermée étroitement dans le fruit. C'est un régétal très abondant en Gambie où M. Famechon en a trouvé des forêts compactes de 3 o à 35 kilomètres de long. C'est une graine grasse de grand avenir. Elle donne une huile solide blanc verdâtre qui est employée par les indigènes pour leur alimentation (1). Le rendement des graines en corps gras est de 40 o o: on en extrait $800 \%$ d'acides gras de distillation et 200 o d'acides gras solides de distillation (stéarique) dont le point de solidification est de 45 degrés. Le tourteau est très utilisable mais commeengrais seulement à cause de sa saveur désagréable. Les bougies faites arec l'acide stéarique sont fort belles. Tous les produits que nous renons d'examiner arec leurs dérivés, acides gras de distillation et de saponification, glycérines, bougies, figurent dans l'Exposition du Musée colonial de Marseille.

\section{IV. - LE TABAC DANS LES COLONIES PAR M. LALRENT.}

M. le Dr Laurent, qui s'est chargé de l'étude des narcotiques coloniaux (Tabac, Opium, Haschich, Bétel, etc), va faire paraitre dans les Annales de l'Institut colonial (1900) une monographie complète du tabac, seule plante économique de très haute importance pour laquelle la bibliographie soit restée excessivement paurre.

Après une étude approfondie des espèces et des variétés botaniques, l'auteur en dehors des questions de manipulations industrielles sur lesquelles il insiste beaucoup, s'est surtout efforcé d'élucider les questions suivantes, qui ont un haut intérèt pour nos colonies,

(1) Cette graine pourrait être obtenue à $10 \mathrm{fr}$. les 100 kilos sur la côte à Konakry: celle de Lamy qui n'exige pas de décortication à $15 \mathrm{fr}$. Les I oo kilos (renseignements dus à $\mathbf{M}$. Famechon, directeur des douanes à Konakry). La quantité que la demande pourrait exiger est assurée par la production spontanée de la plante sans culture. 
savoir : $1^{\circ}$ si le tabac possède en France des débouchés suffisants, $2^{\circ}$ si les colonies remplissent les conditions nécessaires pour l'obtention des qualités supérieures, $3^{\circ}$ quelles sont dans chacune d'elles les règles capitales à observer pour la culture et le traitement des feuilles; et enfin, quel est le mouvement commercial de ce produit dans nos possessions françaises.

On peut dire, que s’il est un produit du solqui a pris de nos jours, dans le monde entier, une prépondérance énorme, c'est le Tabac, puisqu'il est consommé par la totalité du genre humain.

Connu depuis trois siècles en Europe, après aroir été employé de temps immémorial par les populations du centre de l'Amérique, il s"est répandu partout avec une rapidité telle, que dès le $x v 11^{\circ}$ siècle les souverains s"inquiétaient de voir leurs sujets faire usage et abuser d'une herbe dont la fumée, au dire de Jacques 1 d'Angleterre, ressemblait à celle qui s"échappait du gouffre du Styx plein de poix et sans fond.

Pendant une période de cinquante années, comprise entre 1820 et 1870 , la consommation du tabac en France a subi une progression rapide. Elle atteignait à cette dernière date le chiffre de trente millions de kilogrammes et monta peu à peu jusqu'à l'heure actuelle (fin 1898) à plus de trente-sept millions de kilogrammes. Si le ralentissement a été considérable pendant ces dernières années, il ne faut pas perdre de vue que les conséquences de la guerre de 1870 , et le très faible accroissement de la population sont autant de causes qui s'opposent à la consommation d'un produit, dont l'usage est pour ainsi dire limité par le bien-être des peuples civilisés, et. ajoutent des auteurs peut-ètre aussi par leur raison.

Au point de rue économique, quelques chiffres feront voir toute l'importance que cette culture peut avoir dans les colonies, quand, sagement et rationnellement dirigée, elle donnera une récolte abondante. Celle-ci préparée d'après les meilleurs procédés employés en Amérique et en Europe, constituera pour le planteur une branche importante de revenu.

Durant les quatre dernières années, le chiffre de la consommation totale du tabac en France, a oscillé entre 37 et 38 millions de kilo- 
grammes donnant un revenu brut de foo millions et un bénéfice net au budget de 323 millions de francs.

Or, tout le monde sait que le tabac est cultivé dans un certain nombre de départements français sur une assez grande échelle. Non seulement, la production de la mère patrie, telle qu'elle est réglementée de nos jours, est loin d'égaler la consommation; mais encore les tabacs français ne pourront jamais rivaliser avec ceux que l'on obtient dans les pays situés sous les latitudes plus basses; latitudes qui sont précisément celles des grands pays producteurs et des meilleurs crùs du monde entier, et entre lesquelles est comprise la plus grande partie de notre empire colonial.

Or. cette portion qu'elle est obligée de demander à l'étranger, la France aurait tout intérêt à la tirer de son domaine colonial qui apporterait à ses produits non seulement la bonne qualité mais la supériorité d’être français.

- Les cinq contrées qui, sur le globe, produisent le plus de tabac sont : les Etats-L'nis, la Turquie, le Brésil, l'Insulinde hollandaise. les îles Philippines et Cuba dans les Antilles, cette dernière conservant une supériorité incontestable et incontestée sur les autres pays. De là viennent les célèbres tabacs de la Havane, estimés dans le monde entier.

On remarquera en outre. queles meilleurs crùs sont situés dans la zone intertropicale d'où la plante est originaire. C'est dans les contrées de l'Amérique centrale, où la plante s'est naturellement cantonnée qu'elle a acquis au plus haut degré les qualités intrinsèques recherchées des connaisseurs.

Plante annuelle, ne demandant qu'un laps de temps relativement court pour accomplir son cycle régétatif; pourvue d'une force d'adaptation et d'expansion assez considérable, elle a pu ètre acclimatée dans les paýs les plus divers, sur les sols les plus disparates et sous des latitudes assez élevées comme la Hollande, par exemple, ( 520 nord), et donner des feuilles, qui, en apparence du moins, ne le cèdent en rien à celles des pays d'origine. Mais il n'en est rien.

Sicertains caractères botaniques se conservent, si l'aspect, le port de la plante ne varient point, pendant plusieurs générations, sous un 
ciel moins clément. il n’en est pas de même des qualités intrinsèques qui donnent au produit sa vraie valeur.

La supériorité d'un tabac dépend de quatre facteurs, toutes considérations spécifiques égales d'ailleurs, et, de cette supériorité même, dépend nécessairement la valeur commerciale.

Ces facteurs sont: la combustibilité, la finesse du parenchyme, la teneur en nicotine. l'aròme,

Le planteur peut aisément obtenir le premier, en établissant sa culture sur un sol sablonneux, riche en potasse, ou enrichi par l'addition d'engrais appropriés.

Le second dépend aussi de la nature du sol, de la quantité d'humidité répandue dans l'air et de l'époque de la récolte.

Le troisième. plus délicat à faire variẹr, dépend teaucoup du sol, de l'état hygrométrique de l'atmosphère, du climat, d'une façon générale, des engrais employés et aussi de l'époque de la récolte des feuilles.

Quant au dernier, il faut bien l'avouer, l'action du planteur est presque nulle; les causes qui agissent sur lui sont très complexes et ne paraissent être réalisées que dans les pays tropicaux.

Tous les tabacs fins remplissant les conditions qui viennent d'être énumérées proviennent des contrées chaudes. Or tout semble concourir pour indiquer nettement la place que les colonies françaises pourraient prendre dans le mouvement commercial d'un produit qui jadis fit leur richesse. La plupart d'entre elles possèdent un climat bien approprié; on trouve presque dans toutes des sols sablonneux, siliceux provenant de la désagrégation des roches anciennes. Enfin d'une manière générale, les essais qui ont déjà été tentés ont tous donné des résultats favorables.

Tout concorde donc pour assurer à nos possessions une place avantageuse et ce devrait être pour les planteurs un encouragement à y tenter la culture. Pourtant notre domaine colonial ne produit pas une quantité de tabac suffisante pour sa consommation. Pourquoi cela, puisque le sol et le climat conviennent à cette culture? Il faut en chercher la cause dans les mauvaises méthodes de culture et le manque absolu de débouchés dans la mère-patrie. 
L'Angleterre, en prohibant la culture du tabac sur le territoire des Iles Britanniques, a eu principalement en vue le bénéfice que son domaine colonial pourrait tirer de cette plante, et du reste, nous trouvons dans nos législations antérieures des traces non équivoques de tentatives faites dans ce sens.

Dans la première ordonnance du roi Louis XIII, en $\Sigma 629$, qui frappait de trente sols par livre le tabac étranger, il est dit expressément que ce droit sera prélevé « excepté pour celui qui viendra des isles de Saint-Christophe, la Barbade et autres isles occidentales qui appartiennent à la Compagnie formée pour habiter les dites isles; duquel droit nous l'avons déchargé et exempté pour favoriser d'autant plus l'établissement et accroissement de la Comgagnie qui a été dressée pour le bien général de notre royaume. »

Pendant le xv111e siècle, la culture, après avoir été prohibée dans le royaume pour favoriser le commerce extérieur et en particulier celui de nos colonies, fut autorisée dans certaines provinces puis déclarée libre. Enfin. dans le commencement de ce siècle, elle fut monopolisée et permise seulement dans un certain nombre de départements dont le nombre a peu à peu augmenté.

Mais, quoi quil en soit de la production du tabac en France, il n'en reste pas moins un point bien acquis: c'est qu'alors mème qu'en augmentant la superficie cultivée on puisse obtenir, sous les latitudes tempérées, la quantité nécessaire à la consommation intérieure, on n'obtiendra jamais la qualité que l'on recherche avant tout de nos jours dans les produits manufacturés. La France produit des tabacs corsés plus qu'il ne lui en faut. Ceux-ci entrent dans la confection de la poudre et des rôles ou tabacs à màcher. Mais elle est tributaire de l'étranger pour les tabacs fins qu'il semble impossible d'obtenir dans nos climats.

La France récolte annuellement de 25 à 30 millions de kilos de tabac elle en demande 2 à 3 millions à l'Algérie et en achète 15 millions environ à l'étranger. Il semble dès lors anormal, qu'elle ne demande pas la plus petite quantité à ses colonies dans lesquelles le tabac prospère et où assurément il ne manque à sa culture qu'un peu d'encouragement et quelques procédés plus rationnels 
que ceux actuellement employés, pour arriver à donner l essor désírable à une production pour laquelle la métropole est dans une si large part tributaire d'un sol qui ne lui appartient pas.

Algérie. - La culture du tabac est libre dans notre colonie et y occupe un certain nombre de planteurs. On a surtout reproché au produit sa mauraise combustibilité provenant sans aucun doute de la pauvreté du sol en potasse. Chaque bande de terrain possède son clinat propre résultant des courbes isothermiques et hygrométriques et varıant aussi avec l'altitude et l'orientation des vallées. Il subit une double influence, celle de la mer Méditerranée qui rend la région tempérée et celle des vents du Sud qui soumet la région aux changements brusques des climats continentaux.

Les variétés qui paraissent donner les meilleurs résultats sont le Philippin et la variété dite du Chébli.

L’Algérie est la seule de nos colonies d'où le tabac soit exporté. Son commerce donne d'ailleurs lieu à un chiffre assez important d'affaires.

Tabac en reuilles

$$
\begin{aligned}
& 1888-2.300 .000 \mathrm{kilog} . \\
& 1889-1.100 .000- \\
& 1890-2.000 .000- \\
& 1891-2.700 .000- \\
& 1892-2.400 .000- \\
& 1893-3.200 .000- \\
& 1894-2.000 .000- \\
& 1895-3.200 .000- \\
& 1896-3.300 .000- \\
& 1897-5.000 .000-
\end{aligned}
$$

\section{Tabac fabriqué}

$1.300 .000 \mathrm{kilog}$.

Tunisie - En Tunisie, le tabac est monopolisé par l'Etat, la matière première est achetée soit en Algérie soit à l'étranger.

$$
\begin{array}{ccccc}
1893 & 1894 & 1895 & 1896 & 1897 \\
586.938 & 861.628 & 727.902 & 601.945 & 510.796 \\
\text { francs. }
\end{array}
$$


Sénégal. - Bien que le tabac pousse presque partout à l'état sauvage en Cazamance. on ne lui fait subir aucune préparation, il est fumé après avoir été simplement séché.

On a importé en 1896 :

Tabac en feuilles........... $681.493 \mathrm{kilog}$.

Cigares et cigarettes......... $12.402 \quad$ -

Le tabac provient en majeure partie des Etats-Unis. Quant à l'exportation elle est nulle.

Côte occidentale dAfrique, Dahomè: Guinée, Congo. - Le tabac pousse fort bien dans toute cette partie de l'Afrique qui pourrait fournir à la métropole un tabac d'excellente qualité. Les meilleures saisons pour y ensemencer le tabac sont les mois d'octobre et de décembre, mais nulle part le produit ne reçoit une préparation suffisante pour être transformé en produits de bonne qualité. Aussi l'exportation est nulle, et le commerce d"importation est le suivant :

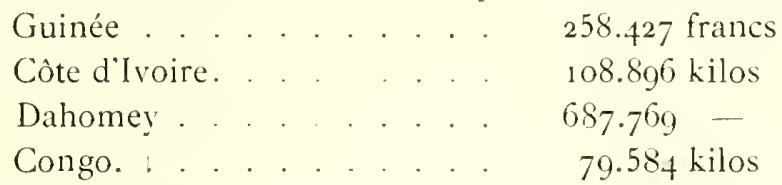

Madagascar. - Le tabac qu'on y cultive est de bonne qualité. Bien préparée, la variété vaut assurément celle de la Réunion mais, comme partout dans nos colonies. le tabac ne reçoit de la part des indigènes qu'une préparation sommaire. Madagascar a reçu de la France. en 1897 , pour 22.000 francs de tabac et près de 300.000 de pays étrangers.

La Réunion. - C'est une des colonies où la culture du tabac est conduite d'une façon rationnelle et dans laquelle la récolte donne des résultats satisfaisants. Bien que cette île reçoive une certaine quantité de cette matière de consommation, l'exportation donne lieu à un assez important commerce.

Elle a exporté il y a trois ans à Naurice 12.00o kilos de tabac en feuilles, 50.000 kilos de tabac haché, ainsi que 10.000 cigares.

\section{COLONIES D'ASIE}

Indo-Chine. - Il résulte des différents essais tentés dans notre possession, que la culture du tabac doit donner des résultats satisfaisants. Grâce à des amendements du sol rationnellement pratiqués et à une préparation méticuleuse du produit. on améliorera de plus en 
plus la culture d'une plante qui est une source de richesses pour les colonies néerlandaises de l'Insulinde; mais, la préparation du tabac ayant une influence considérable sur la bonne qualité du produit, il faut que les planteurs se souviennent que le climatde la Cochinchine les oblige à apporter des soins minutieux à la manipulation du tabac.

En 1896 , le tabac annamite a donné lieu à un chiffre d'exportation très faible (1658 kilogr.)

La même année, il a été importé, en Cochinchine et Cambodge $52+455$ kilogr.

En Annam 1516: kilogr.

Au Tonkin 59243 kilogr.

Le Tonkin a en ontre exporté en Chine 1456 kilogr.

Quant à nos possessions de l'Inde, elles se suffisent à peu près à elles-mêmes.

Le tabac est cultivé dans toutes nos colonies, son développement y est rapide. Mais on doit surveiller d'une manière spéciale le séchage qui s'effectue ordinairement mal, à cause de l'humidité de l'air très abondante dans ces climats similaires tropicaux.

Dans certaines localités, on fait une grande consommation de tabac. Préparé suivant les meilleurs procédés utilisés en Amérique et en Europe, il pourrait donner lieu à un certain mouvement d'exportation, car la culture est facile, et beaucoup de terres océaniennes s'y prêtent admirablement, notamment la Nouvelle-Calédonie où cette culture est très développée à Bourrail, La Foa, Lifou, Nouméa, Poucrabouen.

Quoiqu il en soit la France a importé en 1896 en Nouvelle-Calédonie 21312 kilogr. de tabac et les autres pays, 58908 kilogr.

Quant à Tahiti, on y importe $4 \psi^{62}$ kilogr. de tabac à fumer et I 20 mille cigares et cigarettes, elle exporte dans les îles voisines un millier de kilos environ.

Antilles. - Le tabac fut d'abord cultivé dans nos possessions des Antilles qui en tiraient un assez grand bénéfice; plus tard il fut abandonné pour la culture de la canne à sucre. Maisil ne faut pas perdre de vue que nos colonies des Antilles peuvent livrer à l'exportation un tabac de qualité supérieure et que la culture est une source de bien-être pour la petite propriété. 
L'exportation du tabac est nulle à la Martiniqne et à la Guadeloupe on a importé en 1896 :

\section{Martinique}

Tabac en feuilles $232.643 \mathrm{kilogr}$.

Cigares Cigarettes $59 \mathrm{r} \quad-$

Tabac à fumer 3 I.960 -
Guadeloupe

:09.406 kilogr.

$130 \quad-$

$24.084 \quad-$

Guyane. - Le sol des forêts nouvellement défrichées parait être pour cette culture, un terrain très favorable. Là encore en regard d'une exportation nulle s'inscrivent les chiffres suivants d'exportation: pour l'année 1896 :

$\begin{array}{lrc}\text { Tabac en feuilles } & 27761 & \text { kilogr. } \\ \text { Cigares } & 4181 & - \\ \text { Tabac à priser } & 164 & - \\ \text { Tabac à fumer } & 44129 & -\end{array}$

Ce rapide coup d'œil, montre que tout est à faire pour ainsi dire dans cette voie.

La métropole devrait songer à encourager le colon dans la culture d'une plante universellement consommée qui se développe très bien dans notre domaine colonial, et cependant les colonies ne peuvent se suffire à elles-mêmes puisqu'elles sont obligées d'en demander à l'étranger une quantité considérable. 

II.

\section{Résumé}

\section{DES \\ Travaux Zoologiques}

de I'Institut Colonial de Marseille

dirigé par M. le Professeur HECKEL.

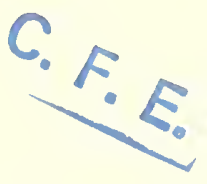





\title{
PREMIER MEMOIRE
}

\section{Contribution}

\section{à l'Histoire Naturelle}

\author{
DES GRYLLIDA \\ Brachytrypes achatinus qui ravage les plantations de cafe \\ an Tonkin

\section{PAR L. BORDAS} \\ Docteur és-sciences, Docteur en médecine \\ Chef des Travaúx de Zoologio à la Faculté des siciences de Marseille \\ Mémoire de 100 pages avee 40 figures intercalés dans le texte.
}

Ce travail est actuellement terminé et paraitra incessamment dans les Annales de l'Institut colonial de Marseille.

Les matériaux de ce mémoire proviennent d'envois divers faits à l'Institut colonial de Marseille et qui nous ont été très obligeamment communiqués par le directeur, $M$. le professeur Heckel, à qui nous nous empressons d'adresser tous nos remerciem ents.

On sait combien sont considérables les dégâts causés par certains Orthoptères (Acridiens) et les pertes énormes, se chiffrant par millions, qu'ils occasionnent chaque année dans la plupart des contrées du nord de l'Afrique, l'Algérie surtout. Jusyu'à présent, on n'avait signalé nulle part, sauf quelques légères déprédations produites dans nos jardins par les Gryllotalpa (courtilières), de ravages 
dus à des Gryllides. Il existe cependant un Gryllide géant, le Brachytrypes, dont la taille atteint jusqu'à 50 millimètres, qui exerce actuellement ses ravages dans les plantations de café de certaines régions du Tonkin. Cet Orthoptère se creuse des terriers, sortes de galeries souterraines sinueuses et irrégulières, coupe les racines, ronge les radicelles et amène ainsi la mort de nombreux plants de caféiers. Les ravages occasionnés par cet Orthoptère sont parfois si étendus qu'ils ont fini par attirer l'attention des agriculteurs tonkinois.

Il était tout naturel de chercher tout d'abord à connaître l'anatomie et l'organisation interne de cet insecte, afin d'aviser ensuite aux moyens à employer pour sa destruction.

Le Brachytrypes, n'ayant pas encore été étudié, nous a présenté une structure fort différente de ses congénères de la même tribu et nous a permis de faire de nombreuses et intéressantes observations scientifiques toutes nouvelles, que nous avons consignées dans le présent mémoire.

Sans négliger le côté pratique, consistant à rechercher les procédés à mettre en usage pour amener une destruction rapide des Brachytrypes et circonscrire ou même arrêter leurs ravages, nous avons soigneusement étudié, au point de sue scientifique, son organisation interne. Ses différents organes: Tube digestif, Glandes salivaires, Tubes de Malpighi, Système neryeux, Organes reproducteurs, Musculature, etc., ont été l'objet d'études histologiques et anatomiques spéciales.

Les Chapitres concernant l'armature buccale, les appendices thoraciques et l'appareil digestif ont été surtout développés avec ampleur, attendu que les organes qui y sont décrits sont, pour l'insecte, les instruments les plus actifs mis par la nature à son service pour exercer ses dépradations. - Chaque description est accompagnée d'une figure représentant l'organe en question.

L'armature buccale comprend le labre, les mandibules, les mâchoires (antérieures et postérieures) et le labrum.

Les mandibules sont chitineuses, courtes, trapues, à base élargie, très fortes et actionnées par de puissants faisceaux musculaires. Leur 
extrémité libre antérieure est recourbée et constitue une dent très acérée. En arrière de celle-ci et du côté interne, existe une surface aplatie et tranchante, suivie de 4 ou 5 denticules coniques. La forte musculature basilaire met en mouvement la mandibule qui fonctionne à la façon d'une scie et peut ainsi couper les fibres ligneuses les plus résistantes.

Les mâchoires antérieures sont caractérisées par la forme et la puissance du lobe interne. Ce dernier est long, recourbé, et représente exactement la forme d'une faucille. Son bord interne est tranchant, son extrémité libre, arquée et pointue porte, vers le quart antérieur de sa courbure, une légere denticulation hémisphérique. Le rôle des mâchoires, agissant à la façon des sécateurs est évidemment de concourir avec les mandibules pour couper et broyer les racines et les radicelles.

L'appareil digestif du Brachytrypes, organe d'herbivore, est remarquable par sa longueur et les nombreux replis que forme l'intestin moyen. - Comme jusqu'à présent il n'a été donné aucune description anatomique ni histologique de cet appareil, nous allons tout particulièrement attirer l'attention sur ses parties principales: jabot. gésier, intestin moyen et tubes de Malpighi.

Le jabot est une vaste poche large, sacciforme, cylindrique en avant, renflée et terminée en cœecum en arrière. Il présente, par sa forme, un terme de passage entre le jabot des Gryllus et celui de la Gryllotalpa. Ses parois internes sont recouvertes d'une mince membrane chitineuse, plissée et portant de fines soies cornées. Un appendice tubuleux, fixé excentriquement et partant de sa région postéroinférieure, sert à unir le jabot au gésier.

Le gésier est un organe globuleux, à parois musculaires très épaisses et recouvertes intérıeurement par six rangées de dents chitineuses, très puissantes, très acérées et implantées perpendiculairement à l'axe. Ces dents font l'objet d'une description spéciale et ont pour fonction de broyer les particules ligneuses ou autres qui ont échappé à la trituration des mandibules et des machoires. L'ensemble des dents du gésier constitue un appareil masticateur 
d'une grande puissance et son mode d'action sur les particules solides s'effectue à la façon d'une râpe.

L'intestin moyen porte, à son origine, deux volumineux cœecums ou appendices intestinaux, de nature glandulaire et entourant latéralement le gésier. Le reste de l'organe est cylindrique, plissé et reçoit, à son extrémité postérieure, le conduit excréteur unique des Tubes de Malpighi.

On sait que les Tubes de Malpighi sont des organes urinaires et représentent l'appareil rénal des insectes ( $V$. notre Mémoire: Tubes de Malpighi des Hyménoptères, in Bulletin Scientifique de la France et de la Belgique, avec trois planches, 1895). Ceux des Brachy-trypes sont remarquables et affectent une disposition anatomique très curiease et non encore décrite. Chez les Grillons, ces organes forment une touffe, s'ouvrant au sommet dilaté du conduit excréteur. Chez le Brachytrypes, le conduit excréteur, qu'on peut comparer à un uretère, se bifurque à son sommet et donne deux volumineux cœcums, longs, cylindriques, sortes de vessies urinaires dans lesquelles viennent déboucher les tubes de Malpighi. Ceux-ci, de couleur blanchâtre, très nombreux, forment un chevelu inextricable et vont déboucher dans les canaux récepteurs fixés au sommet de l'uretère.

Le système nerveux sous intestinal. le système nerveux sympathique, les organes reproducteurs mâles et femelles ont, de même, été décrits et forment autant de contributions nouvelles à l'anatomie entomologique. 


\section{DEUXIÉME MÉMOIRE}

D'autres matériaux d'études, provenant également de l'Institut Colonial de Marseille, ont fait, grâce à l'extrême obligeance de M. le Professeur Heckel, l'objet du mémoire suıvant :

\section{Etude du Fourreau}

\section{d'une Espèce de Psychidé}

\section{DES ENVIRONS DE TOMBOUCTOU ( 1 )}

par MM. VAISSIÈrE,

Professeur à la Faculté des Sciences de Marseille

et le

Docteur L. Bordas

Ghef des travaux pratiques de zoologie

Les auteurs, grâce aux variés et très nombreux échantillons fournis par le Musée colonial, ont pu, non seulement étudier le fourreau du Psychidé, mais encore faire l'anatomie de son parasite, une larve de Braconide. Ils ont, en outre, par l'examen de la disposition présentée par l'hôte et le parasite, ajouté une page intéressante de plus à la biologie, si peu connue encore, du parasitisme chez les insectes.

On sait que parmi les Braconides, l'A panteles glomeratus, par exemple, dépose ses œufs sur le corps des chenilles de la Piéride du

15 pages, I pl. et 15 figures dans Ann. de la Faculté des Sciences de Marseille T. X. $\mathbf{} 899$. 
chou. Les chenilles infestées par l'A panteles sont très nombreuses et atteignent leur taille normale, mais prennent une coloration jaune pâle, ce qui permet de les distinguer de celles qui sont saines. Parfois aussi, les œufs sont introduits dans l'intérieur du corps de l'hôte (Rhinocyllus latirostris) et on assiste alors à des phénomènes de parasitisme interne.

Or, certains fourreaux de Psychidés soumis à notre étude renfermaient des larves de Braconides. Dans la coque nymphale se trouvaient, entourés par un épais feutrage de filaments blanchâtres, de nombreux œufs, de couleur jaunâtre et à coque dure et cornée. Certaines chenilles de Psychidés ont, à leur sortie de l'enveloppe nymphale, le corps couvert de longs poils cylindriques. Les femelles de ces espèces ne quittent pas leur fourreau, mais présentent, à l'orifice inférieur de celui-ci, l'extrémité postérieure de leur corps pour permettre aux mâles de les féconder. La fécondation accomplie, elles retournent au milieu de leur habitation, s'entourent de nouveau de leur enveloppe nymphale et ne tardent pas à mourir, servant ainsi de pâture à leurs œufs. Ceux-ci se développent rapidement, abrités par les téguments maternels et par ceux de la nymphe. Les œufs, après avoir augmenté de volume, se trouvent bientôt enveloppés par les faisceaux de poils qui entouraient le corps de la mère. C'est dans la coque nymphale que se développent les larves parasites de Braconides étudiées au cours du Mémoire. 


\section{TROISIÈME MÉMOIRE}

\section{Les Acridiens}

ÉTUDES ANATOMIQUES ET BIOLOGIQUES, DÉTELOPPEMENT, MIGRATIONS DÉGATS CONSIDÉRABLES QU'ILS GAUSENT A L'AGRIGULTURE MOYENS EMPLOYÉS POUR LES COMBATTRE, PAR M. Bordas.

Mémoire de 200 pages et 80 figures, en préparation et pour paraître prochainement dans les annales de l'Institut Colonial de Marseille.

L'auteur, bien préparé à ce genre d'études par de nombreux travaux scientifiques sur les Orthoptères, (I) a largement mis à contribution les richesses entomologiques que possède l'Institut colonial de Marseille. De plus, il a voulu se rendre compte par lui-mêrne, en visitant les lieux dévastés, des dégàts considérables occasionnés par certains Acridiens (Criquets). C'est ainsi qu'en août et septembre I 899, il a parcouru le nord de l'Algérie et de la Tunisie et en avril I 900 , il a fait un second voyage dans la Kabylie et aux environs de Bougie, de Beni-Mansour et Sétif.

De nombreuses notes ont été prises qui, ajoutées aux résultats de recherches anatomiques et histologiques poursuivies actuellement, formeront dans quelques mois l'histoire naturelle complète des Acridiens.

(1) V. L. Bordas. - L'appareil digestif des Orthoptères. Mémoire de 250 p. XII Pl. et ، 30 fig. : Annales des Sciences Nat. Zool. - $8^{*}$ série. T. V. 1897 



\section{NOMENGLATURE}

des produits exposés par l'Institut Colonial avec les indications du groupe et de la classe auxquels ils appartiennent:

Nọ 1. - PRoduits agricolles Alinentaires d'origine VéGÉTAle

Gomme de Cupania, comestible chez les Canaques. G. 12 C?.87

Fruits et graines de W'Bentamaré ; Cassia occi-

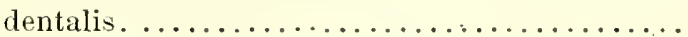

Gafé Nègre de M'Bentamaré, Cassia occidentalis.. Mengoun, graines comestibles, Dalbergia macros.

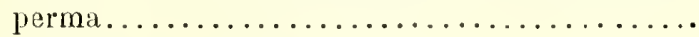

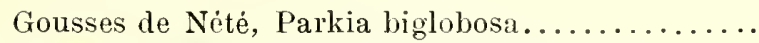

Gomme de M'Beppe, Sterculia tomentosa..........

Fruit de Can-Thau, Feronia elephantum.........

Fruit de l'arbre du voyageur, Ravenala Madaseariensis.

Gousses de vanille sauvage, Vanilla phalenopsis... Fleur

\section{No 2. - PRODUITS AGRIGOLES NON ALIMENTAIRES}

Graines grasses nouvelles ou peu connues des colonies francaises avec leurs dérivés (acides gras, corps gras, glycérines, savons, tour teaux, bougies). - Gommes, gommes lésines et résines des colonies françaises.

Risine fossile d'Araucaria Cooki............ - C.8.87

Gomme résine séche d'Araucaria Cooki......... - -

Gornme résine d'Araucaria Cunninghami....... - -

Risine

Gomme résine d'Araucaria Brasiliensis...........

Gommme résine d'Araucaria excelsa.......... - -

Gomme d'Araucaria liulei ............... - -

Gomme résine d'Auraucia Riulei........... - -

Résine d'Araucaria Rulei................ - -

Bois de chène gomme, spermolopis tanuiferá, avec

Tanno-résine de chène gomms, Spremolep:s tanni-

fera 
Bois d'absinthe, Beilschmiedia anisiodora........

Bois chandelle, Myoporum tenuifoliun...........

lićsine fossile de Kaori blanc. Dammara ovata.....

Résine fossile de Kaori rouge, Dammara Moorei. . Porte-cigares, porte-cigarettes, bouts de pipes en résine ambre de Kaori rouge................ Bois d'Erouma, arbre à laque, Macaranga Vedeliana Huile de Cerber a Manghas................. Fruits et graines de Cerbera Manghas........... Gomme rẻsine d'Araucaria Bidwilli............. Bloc de bourgeons résineux de (tardenia Aubryi... Glycérine de Cerbera Nanghas................ Acire gras de saponification de Cerbera Manghas. . Acirle gras de distillation de cerbera Manghas..... Stéarine de saponification

Stéarine de distillation

Bois de Fontainea Pancheri (Heckel)............

Feuilles de Mairé, Alixia stellata...............

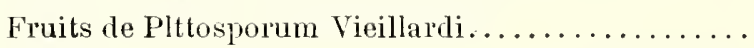

Résine

Gomme laque de Lokomhitsica, Gascardia Madagascariensis.

Graines de D'javê ou N'javé, Baillonella toxisprer'ma Beurre

Acide gras de saponification de D'javé

Acide gras de distillation de D'javẻ ou N'javé, Bail-

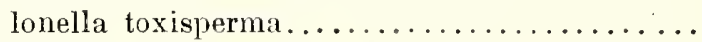

Stéarine de saponification de D'javé ou N'javé,

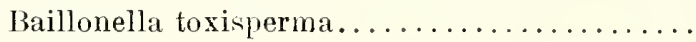

Stéarine de distillation de D'javé ou N'javé, Baillo-

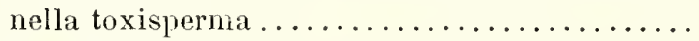

Glycérine de distillation de W'javé ou N'javé, Baillo-

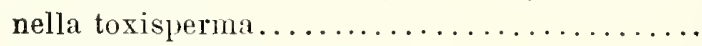

Graines de Karité, Bassia Parkii............. Beurre dè Karité

Graines de Soumpé, Balanites Egyptiaca....... Huile de Soumpé

Copal de Casamance, Guibourtia copalifera. 
Graines de Méné, Lophira alata.

Huile de Méné

Acide gras de saponification

Acide gras de distillation -

Glycérine de Méné

Stéarine ie saponification -

Stéarine de distillation

Bougie de Méné

Toưrteau de l'amande de Iéné

Graines d'Oba, Irvingia gabonensis

Pain d'O'Dika

Beur'e d'O'Dika

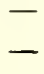

cristallisé dans l'alcool....

cristallisé dans l'acétone. .

Acide gras d'O'Dika, Irvingia gabonensis........

Graines d'Ongueko, Ongokea Klaineana..........

Huile d'Onguéko.

Acide gras saponification d'Onguéko

Fruits de New, Parinarium senegalense.........................

Huile de New,

Acide gras saponification de New -

Acide gras distillation de New

stearine saponification dr New -

Stearine distillation de New

Glycérine de $N{ }^{2} w$

Graines d'Owala, Pentachethra macrophylia.......

Huile d'Owala

par éther do petrole

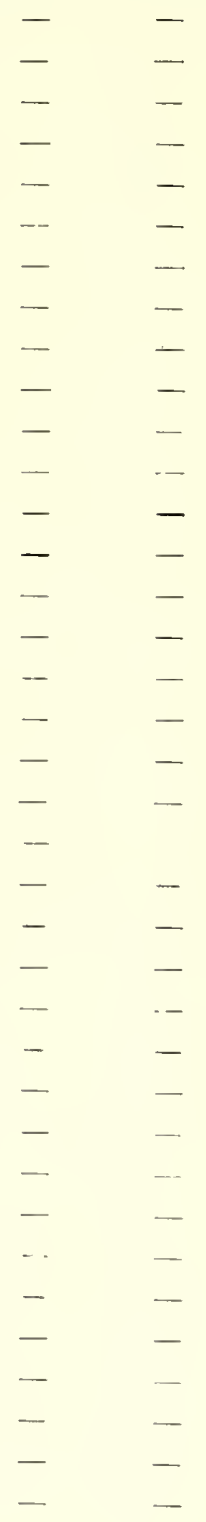

Acide oléique dowala, Pantuclethra macrophilla. .

Stéarine d'Owala

Bougies d'owala

Tourteau d'Owala

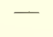

Graines de Lamy, Pentadesma lutyracea ........

Beurre de I amy

Stearine de distillation de Lamy

stéarine de spanification de Lamy

Acide gras de saponification de Lany - .......

Acide gras de distillation de Lamy 
Glycérine de Lamy

Graines de Maloukang. Polygala butyracea....... Beurre de Maloukang

Acide gras de saponification de Maloukang... .. .

Acide gras de distillation de Maloukang.........

Stéarine de saponification de Maloukang.........

Stéarine de distillation de Maloukang ..........

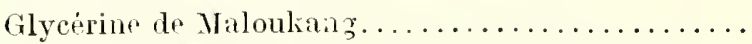

Graines de Makeron; Tieghemella Heckeliana....

Co:ps gras de Makerou

Acide gras de saponification de Nakerou - .....

Fruits et graines de Kombo; Pyenanthus microce-

phalus.

Beurre de Kombo

Acide gras de distillation de Kombo

Graines d'Ochoco; Ochocoa Gaboni.

Beurro d'Ochoco

Corps gras d'Ochoco - parther de pétrole

Acide gras d'Ochoco

Fruits de Badamier, Terminalia Catappa........

Huile de Badamice

Acide gras de saponification Batlamier $-\ldots \ldots$.

Acide gras de distillation du Badamier - ......

Palmitine de saponification du Barlamier - ......

Palmitine de distillation du Badamier $\quad-\ldots \ldots$.

G!ycérine du Badamior

Fruits de Kokum; Garcinia indiea

Brurre de Kokum

Acide gras de saponification du Kokum - ......

stéarine de saponification; Kokum, Gareinia intica

Stéarine de distillation de Kokum

Noix de Cay-Cay, Irvingia Oliveri............

beurre de Gay-lay

Acide gras de saponification du lay-Cay -

Acide gras de distillation du Cay-Cay

Risine Chaï, Shorea Thoreli

Graines de Krébao; Hydnocarpus antelminthica.... 
Corps gras de Krébao

Acide gras desaponification de Krébao -

Acide gras de distillation de Krébao -

Stéarine de distillation de Krébao

Stéarine de saponification de Krébao

-

Fruits et graines ie Caïmitier, Chrysophyllum Caimito

Feuilles de Vallanga, Feronia elephantum

Huile de Feronia elephantum.

romme de Velan piciny

Fruits de Staudtia Kamerunensis.

Corps gras de Staudtia -

Graines de Porasum, Butea frondosa.

Huile de Porasum

Acide gras de saponification de Porasum - ....... -

Acide gras de distillation de Porasum

téarine de saponification de Porasum

Stéarine de distillation de Porasum

Glycèrine de Porasum

-

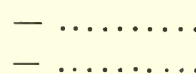

$-$

Graines de Hongay, Pongamia glabra............

Huile de Hongay

Acide gras desaponification de Hongay........... -

Stéarine de saponification de Hongay, ............ -

Craines de bois d'oiseau, Tetranthera laurifolia...... -

Corps gras brut debois d'oiseau

Acide gras de saponification de bois d'oiseau - $\ldots$ -

Acide gras de distillation de bois d'oiseau $\quad-\ldots$ -

Stéarine de distillation de bois d'oiseau $\quad$ - $\ldots$.

Gomme d'acajou, Anacardium occidentale.........

Graines de Sapotillier, Achras sapota............ -

Résine de Gommart, Bursera gummifera......... -

Résine de Gommier blane; Dacryodes hexandra...... -

Résine de Courbaril, Hymene Courbaril........... -

Graines d'Enyessan, Ricinodendron Heudelotii....... -

Huiles d'Enyessan

Acide gras de distillation d'Enyessan

Huile d'Enyessan par sulfure de carbone

$-\quad \cdots-$

Graines d'Elozy-Zẻgué, Ximenia gabonensis......... Corps gras d'Elosy-Zégué - 
Acide gras de saponification d'Elosy-Zégué.......

G. 14 C. 87

Acide gras de distillation d'Elosy-Zégué

Stéarine de saponification d'Elosy-Zégué -

Stèarine de distillation

Graines d'Odjendjé Guassia gabonensis

Corps gras d'Odjendjé

Acide gras de distillation d'Odjendjé

Acide gras de saponification d'Odjendjé ou Quassia

gabonensis

Graines de Boandjo, allanblackia floribunda

Corps gras de Boandio, Allanblackia floribunda....

Acide gras de saponification de Boandjo -

Acide gras de distillation de Boandjo $\quad$ - ...

Stéarine de ${ }^{-}$saponification de Boandjo $\quad-\ldots$.

Stéarine distillation de Boandjo

Glycérine de Boandjo

Tourteau de Boandjo

Graines de Carapa, Carapa guianensis............. -

Huile de Carapa, - $\quad$ -

Corps gras de Carapa $\quad-\quad \ldots . \ldots . . .6$ -

Acide gras de saponification de Carapa guianensis -

Acide gras de distillation de Carapa

Stéarine de saponification de Carapa

Stéarine de distillation de Carapa

Glycérine de Carapa

$-\cdots+-$

- $\ldots$ - -

Fruits de Maripa, Attalea spectabilis........... - -

Huile de Maripa. $\quad$ - $\quad$........... -

Acide gras de saponification de Maripa spectabilis. -

Acide gras solide par expression de Maripa spectabilis _ -

Corys gras de l'enveloppe coque Maripa - $\quad-$

Acide gras de l'enveloppe coque Maripa - $\quad$ -

Fruits de Maripa; Attalea excelsa............. - -

Beurre de Maripa, $\quad-\ldots \ldots \ldots \ldots \ldots$ -

Fruits l'Aouara, Astrocaryum vulgare.......... - -

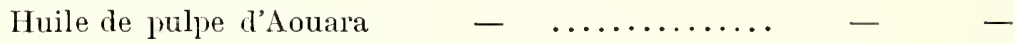

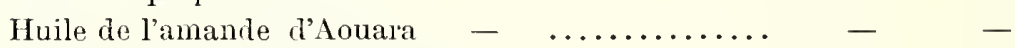

Corps gras de l'amande d'Aouara............. - -

Corps gras de l'enveloppe d'amande Aouara ....... - 
Acide gras de distillation huile amande Chaouari....

Acide gras de saponification enveloppe amande Aouara

Acide gras de saponitication amande Aouara........

Graines de sablier; Hura crepitans..............

Huile de sablier

Acide gras de saponification de sablier.............

Bois dentelle, Lagetta lintearia ..................

Beurre de Pekea, Cayocar butyrosum............

Huile neutre d'amande de Chaouari..............

Huile neutre de coques de Chaouari $-\ldots . . \ldots$.

Acide gras de saponification coques Caryocar

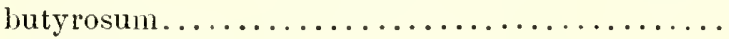

Acide gras de sapenificationamande Chaouari -

Stéarine de distillation huile amande Chaouari -

Stéarine de saponification amandes Chaouari -

Graines de caoutchouc, Hevea guyanenis..........

Corps gras neutre d'Hevea

Acide gras de saponification

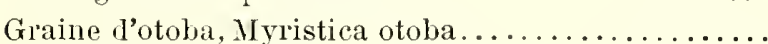

Beurre d'Otoba,

Graines de Guingamadou, Virola sebifera.........

Beurre brut de Guingamadou

Beurre purifiẻ de Guingamadou

Corps gras du macis de Guingamadou ............

Acide gras de saponification de Guingamadou - ...

Acide gras de distillation de Guingam ndou $\quad$ - ...

Graines de Muscadier sauvage, Virola Micheli......

Corps gras, d'amande de Muscadier sauvage

Acide gras de saponification de Muscadier sauvage...

Graines d'Omphalier, Onphalea diandra..........

Huile d'Omphalier'

Acide gras de saponification do l'Omphalier diandle

Fruits de Putranjiva Roxburghii...............

Graines de Polanisia viscosa................

Huile de Polanisia viscosa...................

Acide gras saponification de Polanisia viscosa.......

Graines d'Elengi; Mimusops elengi. 
Huile d'Elengi

Acide gras de saponification d'Elengi.............

G. 1/4 Gl. 87

Acide gras de distillation d'Elengi $-\ldots \ldots \ldots$.

Stéarine de saponification d'Elengi - .........

Stéarine de distillation d'Elengi $\quad-\quad \ldots \ldots \ldots \ldots$

Glycérine d'Elengi

No 3. - instrunients pour la saignée Des arbres a gonme et a résine

Instruments nouveaux pour la saignée des Araucarias

G. 9 Cl. 54

$$
\text { No 4. - Librairie }
$$

Collection complite des Annales de l'Institut colonial

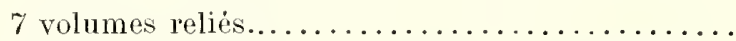

Collection complete des Annales de la Faculté des

G. 3 Cl. 13

sciences de Marseille, 11 volumes reliés....... -

No 5. - arts chimiques et pharmacie. - médicaments des colonies Frangaises D'ORIGINE VÉgétale ET LeURs dérivés

MatiÈrEs TANNANTES ET ÉGORGES TANNANTES

Vernis alcoolique à la résine d'Araucaria Cooki ....

G. 14 Gl. 87

Vernis gras à la résine d'Araucaria Cooki.........

Gateau de résine d'Araucaria Cooki............ - -

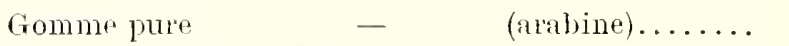

Essence de résine d'Araucaria Gooki............. -

Gomme pure d'Araucaria excelsa (arabine)........ - -

Essence de bois chandelle, Myoporum tenuifolium. _ _ _ -

Gutta de Cerbera Manghas................. - -

Résine d'Erouma, Macaranga Vedeliana........ - -

Résine d'Eucalyptus viminalis (Kino).......... - -

Latex solidifié du Rhus atra............... - -

Huile drastique de Fontainea Pancheri......... - -

Essence do Santal. Santalum austrocaledonicum... _ - _ -

Essence ne citronelle, faux santal, Myoporum cras.

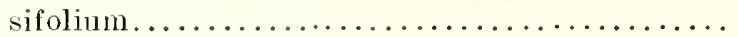

Essence de Thé de Lifou, Limenia minuta........ _ _ -

Essence de thenopodium ambrosioides......... - -

Résine acroïde de Xanthorrea arborea......... - - 
Gutta de D'javé ou N'javẻ, Baillonella toxisperma.

Ecorce de Taly, ou T'ely; Erythrophlœum Guineense.

Caoutchouc de Toll, Landolphia tomentosa ........

Hammout, Balsamo dendron-africanum...........

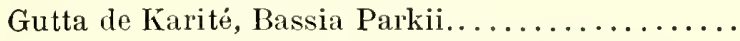

Feuilles de Kinkelibah, Combretum Raimbaulti...

Feuilles de Barsanga, Murraya kœnigi..........

Feuilles de Murraya exotica... ................

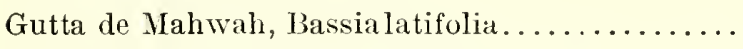

Gutta de Sapotillier (chicle), Achras sapota........

Racine d'Iboga, Tabernanthe Iboga.............

Caoutchouc de Guidroa, Mascarenhasia velutina....

Caoutchouc de Piralahy, Landolphia Perieri .......

Feuilles de Fambleau caraibe (nouveau Jaborandi)

Pilocarpus racemosus.

Ecorce de gommier blanc, Dacryodes hexandra.....

Feuilles de bois d'Inde, Myrtus pimenta..........

Fruits et rameaux de faux poivrier, Schinus Molle.

Essence brûlée de faux poivrier

Essence rectifiée de faux poivrier

Graines de bois d'Inde, Myrtus pimenta.........

Essence de bois d'Inde

Caoutchouc de Dop, Ficus Vogelii............. 



\section{LISTE}

DES

\section{DONATEURS DU MUSÉE COLONIAL}

\section{DONATEURS EN ESPËCES}

MM.

\section{ZARIFI}

Grec

Négociant.

Chambre de Commerce de Marseille.

Cie des Transports maritimes.

Gie Paquet.

Fritsch Estrangin

Français Négociants.

Mante, frères et Borelli

VAGLIANO

Gie du Gaz

Gairard

André et Lieutier

Société Marseillaise

Mallen, Théric et Cie

J.-CH. Roux

$\begin{array}{cc}\text { Grec } & - \\ \text { Gie Française } & - \\ \text { Français } & - \\ - & - \\ - & \text { Maison de banque. } \\ - & \text { Négociants. } \\ - & \text { Industriel. } \\ \text { Grec } & \text { Négociant. } \\ - & - \\ - & - \\ \text { Français } & \text { Négociants. } \\ - & - \\ - & - \\ \text { - } & - \\ \text { Grec } & \text { Industriel. } \\ \text { Français } & \text { Professeur ì la Fa- } \\ - & \text { culté. } \\ - & \text { Industriel. } \\ - & \text { Banquier. }\end{array}$

Etienne Ralli

PANDÉly

ArgentT I

Martin, frères

Mme Veuve Morel

Gie Nationale de Navigation

SOCIÉTÉ IMMOBILIẺRE MARSEILLAISE Th.-Em. Rodocanachi

A. Armand

Charve

Gie des Messageries maritimes

Arnavon

Pascal

Banquier. 


$\begin{array}{ll}\text { Rodocanaghi } & \text { Grec } \\ \text { Bohn } & \text { Français }\end{array}$

Société de L'AFRIQUe ocGidentale Robin, Rondel

Angst

VÉzIAN

Boude

Raffinerie de suare de la méditerranée.

Rodrigues

Scaramanga

Gounelle

Le Petit Marseillais

WaLLER, frères

Allatini

Falgueirettes et Roche

RoLlandin

Cie DEs Docks

Michel Ferrié

Couve

Félix Abram

Gréditt L yonnais

Picon, aîné

Ducreux

Brasserie de la Méditerranée

Morigelly

Gie Fraissinet

Gharbonnages des Boughes-du-Rhône.

Rols et Giraud

J. Digne et Gie

Sermant

Delassus

Planche

G. VIDAL, d'Arles

EberLin

ICARD

Ripert, frères

Pharmacie Gentrale de France
Négociant.

Dr Gie Af rique occidentale.

Négociant.

Banquiers.

Consul du Brésil.

Raffineur de soufre

Banquier.

Négociant.

Industriel.

Journal quotidien.

Minotier's.

Grec Négociant.

Français

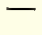

-

$-$

$-$

$-$

$-$

$-$

Francais

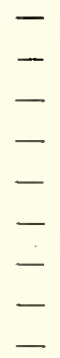


RAYBaUd

Bergasse

LAMOUREUX

VAYSON

Bessède, fils

VERMINCK

Amlé Couve

BARTHÉLEMY

SCHLEEING

SAMAMA

Emile Arnaud

Costa de Glozel et Costa

Ricard

A. Ferraud

RAFFinerie DE SUGRe SAINT-Louis.

Roux Martin

Agelasto

Meynadier

Chanal

Reggio Algibiade

Bellanger et Gervais

MOULIN

H. Rossolin

Nugues, père

Nugues, fils

Ambanopulo

Alexander

Bonnasse

Edm. Puget

Autran, frères

Peytral

Alby, ainé

MaCÉ de Lépinay

\begin{tabular}{cl}
- & \multicolumn{1}{c}{-} \\
- & Armateul. \\
- & Agent de change. \\
- & Rentier. \\
- & Négociant. \\
- & - \\
- & Avocat. \\
- & Président Tribunal \\
& Commerce. \\
- & Industriel. \\
Grec & Négociant. \\
Francais & Minotier. \\
- & Négociants. \\
- & $\quad-$ \\
- & Président dela Chambre de \\
& Commerce.
\end{tabular}

Arbitre de Commerce.

Négociant.

Français

- Industriel.

- Tailleurs.

Minotier.

Rentier.

Minotier.

Grec

Suédois

Franȩais

Négociant.

Courtier maritime.

Banquier.

Négociant.

- Tanneurs.

- Dėputé.

- Negociant.

- Professeur à la Faculté. 
Naegely et Cie

- Bazin et cie

Société DU Rio-'Tinto

Brissonnet, de Tours

Labadí́

SANTI

Moullot

CAYOL

J. Deville

REY

Nicolas, de Castelnau

Darier, de Rouffio

OPPERMAN

PÉRot

Le Comptoir d'Escompte

Cyprien Fabre

Ia Bangue des Chemins de fer CAMPREDON

L.-M. FOURNIER

F. Fournier

JULLIEN

FLACH

SOCIÉTÉ POUR LA DÉFENSE DU COMNERGE.
Nẻgociants.

Industriel.

Pharmacien.

Ancien député.

Opticien.

Imprimeur.

Négociant.

Dr Société Marseillaise (banque).

Négociant.

Industriel.

Ingénieur en chef des Mines.

Prof. à la Faculté.

Banque.

Armateur.

Socièté de crédit.

Nègociant.

Fabricant bougies.

Tanneur.

Négociant.

Socièté de crédit. 


\section{LISTE}

DES

\section{DONATEURS DU MUSÉE COLONIAL}

\section{DONS EN NATURE}

M. Geoffroy, français, pharmacien des colonies: Histoire naturelle Guttas (Mission Guyane).

M. Le colonel Pelletier, français, colonel infanterie marine : Bois armes, ethnographie et histoire naturelle de: Calédonie, Chine, Tonkin.

M. LE Dr $\mathrm{J}_{\mathrm{H}}$ Bulsson, français, médecin des colonies: Coquilles marines et terrestres (de Tahiti et Nouvelle-Zèlande).

M. Castellan, français, capitaine au long cours: Vases, histoire naturelle (Madagascar).

M. Autrearn, français, commis direction de l'Intérieur: Histoire naturelle et botanique (Gabon).

M. AсHARD, français, directeur jardin colonial: Histoire naturelle (Pondichéry).

M. Collard, français, vice-président:Herbier, bois (Cochinchine).

M. Collardeau, français, directeur jardin botanique: Herbier (Guadeloupe).

Reverend père Duss, français, professeur collège : Herbier (Guadeloupe).

Dr Delassus, français, médecin colonies: Balafon, instrument de musique (Soudan).

M. Dupuis, français, Commissaire du gouvernement: Tissus, broderies, armes, orfévrerie, vannerie (Laos). 
M. Foret, francais, administrateur colonial: Histoire naturelle (Congo),

M. Gouson, français, chef d'exploration : Caoutchouc et défenses d'hippopotames (Cungo).

M. Hayes, français, agent de colonisation: Guttas-textiles, gommes résines (Cayenne).

M, JANnEney, français, agent de colonisation: Bois, herbier (Nouvelle-Calédonie).

M. Neveu, francais, directeur jardin botanique : Herbiers, (SaintDenis, Réunion).

M. Prince, français, pharmacien ìes colonies: herbiers (Madagascar).

M. LE Dr Rangon, français, médecin principal des colonies: Gẻologie, histoire naturelle, ethnographie (mission au Soudan).

$D^{r}$ Vincent, français, médecin des colonies : herbier (Tahiti).

Crédit Foncier Colonial, français, société industrielle: Sucres, cafés essences, rhum, subst. alimentaires (Réunion).

M. Georges Perrier, français, importateur: fibres textiles exotiques (Madagascar, Indo-Chine).

M. Ravel fils, francais, importateur: Produits divers (Madagascar).

M. Servel et Cie, français, importateur: Caoutchouc (Madagascar).

Soctétéla Ramie Frangaise, français, industrielle: Collection tissus, fils, itoffes en ramie.

M. L'ABвÉ BoLo, francais, vicaile général: Champignons, carottes, tabac (Réunion).

M. F. Bouquet, francais, planteur: Vanilles (Réunion).

M. Calixte Raibaud, français, néğociant: Bracelet d'argent, d'ivoire des peuples Gallas (Obock).

M. N. BLot, français, directeur d'imprimerie: Tête d'hippopotame (Congo).

MY. LE MEE frères, français. industriels: Beurre de coco alimentaire.

Dr Rovgier francais, médecin messageries : collection bois (Calédonie)

Mlles PAyer, françaises, rentières : Paille chouchouxetobjets fantaisies (Réunion).

M. Julien Leivtitz, français, distillateur : Essences, vins, ananas (Péunion). 
M. Duveau de Vaulcomte, français, planteur: Collection cafés (Réunion).

Socrété La Rivière des Roches, français, planteur : Manioc, tapioca (Rẻunion).

Ve Ch Isautier et fils, français, distillateur: Fissences, rhum blanc, liqueurs (Réunion).

M. Roussel, français, planteur: Collection cafés (Réunion).

Dr Sinon, français, médecin principal colonies: Herbiers, minéraux (Tonkin).

M. Bouéry, français, ingénieur: Caoutchouc (Guinée française).

M. Landréau, français, chef de poste : Ethnographie (Congo).

M. Roraud, français, administrateur : Minéraux (Gabon).

M. Larché, français, administrateur : Minéraux (Nouvelle-Calédonie).

M. Reutier et Cie, français, négociant: Eau-de-vie canne, rhum (Port-Louis, Guadeloupe).

MM. J. G. et P. GÉrard PĖRE, français, négociant: Rhums (Guadeloupe).

M. P. Cordonnier, français, nègociant: (Rhums (Pointe à Pitre).

I. E. Grrard, français, négociant: Rhum (Guarleloupe).

M. Gaston Dagomel, francais, négociant: Rhum (Guadeloupe).

MI. Dubost FRĖREs, français, négociant: Rhum (Guadeloupe).

M. E. Sougues ET Gie, francais, négociant: Sucres, tafias, rhum (Guadeloupe).

Hértters Bonnet, françis, négociant: Tafia (Guadeloupe).

M. G. CAXroL, francais, négociant: Rhum (Guadeloupe).

MM. G. Blongourt et E. Elisée, français, négociants : Tafias (Guadeloupe).

M. H. Lougueteau, francais, négociant: Tafias (Guadeloupe).

II. Olive Guyot, français, négociant : Rhum (Guadeloupe).

M. Albert Vachter et Cre, français, négociant: Rhum (Guadeloupe).

M. A. Gux, franȩais, négociant: Tafias (Guadeloupe).

MM. G. Ballly et.J. LesalNt, français, négociant: Absinthes amères (Guadeloupe).

I. Degrange, francais, négociant: Liqueurs variées aux fruits coloniaux (La Basse-Terre).

Mugee colonial de Harlea, Hollande : Quinquina cultivés (Java). 
M. Ch. Marchand, français, capitaine d'artillerie marine, : (Ethnographie (Madagascar).

M. Henri Dupré, français, négociant: Collection de poivres et cafés.

M. Manasi, français, employé des messageries : Gutta de l'Isonandra (Java).

Chambre de l'agrigulture de la Guadeloupe : Bois (Guadeloupe).

M. Catelan, français, directeur de la Santé: Amphores phéniciennes.

M. DE SaInt-Quentrin, français, trésorier des invalides de la maririe: Chapeaux annamites (du Tonkin).

Société du Rio-Tinto, industrie : Vernis et gommes d'Araucarias.

M. Route, français, fonctionnaire des postes au Laos : Ethnographie (Annam et Cambodge).

Dr Trucy, francais, mérlecin principal des Colonies: Histoire naturelle, Cochinchine. Photographie.

Dr Reroul, français, médecin des Colonies: Plantes médicinales (Soudan).

M. Victor Rouvien, français, consul de Portugal : Collection d'armes et plantes vivantes (Madagascar).

M. Charles Bélanger, français, ingénieur des mines: Minéraux, coquillages. Photographies (Nouvelle-Galédonie).

M. P. H. RAybaud, franȩais. capitaine de port : Histoire naturelle (Sénégal).

M. Colombet, français, garde-magasin colonial: Histoire naturelle (Sénégal).

M. Blarse, français, lieutenant de vaisseau: Ethnographie, Pirogues (Congo).

M. de Boulllane de Lacoste, français, capitaine d'infanterie: Riche collection de bijoux en argent (Laos).

M. Moutin, français, inspecteur de la garde indigẻne : Diplômes mortuaires de Boudahs (Tonkin).

M. Le Dr Rernaud, français, médecin en chef des Colonies : Album botanique de la (Réuuion).

M. GazALBou, français, vétérinaire militaire, agent d'agriculture colonial : Plantes (Soudan),

M. BLot, francrais, vétérinaire militaire: Plantes (Soudan).

Dr Glarr, français, médecin des messageries : Ethnographie et histoire naturelle (Madagascir). 
Dr LAFont, français, médecin des colonies: Ethnographie (Djibouti).

M. F. EnRIch, français, ex-commissaire des paquebots-poste : Collection de fruits exotiques en céramique (Indo-chine).

M. Raoul, français, pharmacien en chef des Colonies : Collections diverses, legs Madinier, bibliothèque coloniale.

M. P. Boude, français, chef du service forestier : Collection des bois du (Cambodge).

M. Rudier, français, pharmacien : Collection de cannes bois (Martinique).

Dr Garnier, français, médecin de marine : Ethnographie (Guyane).

M. LE Fol, français, chef service colonial : Bloc de 50 kilos cristal roche (Diègo-Suarez).

M. H. Toubeau, hollandais, consul des Pays-Bas: Bois de Tech (Java)

M. DE MondÉsIr, français, commandant du Génie : Ethnographie et bois (Madagascar).

M. Edmond André, français, aide-commissaire des Colonies : Ethnographie (Madagascar).

M. Jules Veyan, français, négociant : Ethnographie instruments de musique (Congo).

M. LE D: QueIreL, français, médecin en chef des hopitaux : Ethnographie.

M. Edmond Couve, français, juge d'instruction : Ethnographie.

M. Rossi, français, commis des Colonies: Ethnographie (Afrique).

M. Théodore, francais, commissaire des colonies : Armes du hautOubangui (Congo).

M. C. Bénévent, français, administrateur colonial : Histoire naturelle (Madagascar).

M. Louis Laffite, français, commis des douanes et régies : Histoire naturelle (Congo).

M. Lours Cantet, français, marbrier: Collection de marbres d'algérie et onyx ouvrés.

M. Emile Rolland, francais, comptable: Fossiles des phosphates d'Algérie.

M. Cantini, francais, marbrier : Collection de marbres et onyx d'Algérie ouvrés.

M. DAHER, français armateur: Collection de Phosphates d'Algérie et

Tunisie.

M. Charles Rolland commis de Douane. Fossites des Phosphates. 


\section{NOMENCLATURE DES TRAVAUX PUBLIÉS \\ PAR MM. HECKEL ET SCHLAGDENHAUFFEN,}

dans les dernières années en dehors des Annales

I $^{0}$ du Doundaké et de son écorce, dite quinquina africain, mémoire couronné par l'académie des sciences prix Barbier en 1885 . Octave Doin éditeur.

$2^{0}$ Les kolas africains, monographie botanique, chimique, thérapeutique et pharmacologique (emploi stratégique et alimentaire : commerce) 1893 . Société d'éditions scientifiques.

$3^{\circ}$ Sur les végétaux qui produisent le beurre et le pain d'o'dika, du Gabon Congo et sur les arbres producteurs de la graine et du beurre de cay cay, de Cochinchine et Cambodge, valeur comparée de ces deux produits.

$4^{0}$ Sur le bakis (Tinospara, bakis, miers) et le sangol (cocculus laceba) du Sénégal et du Soudan.

$5^{\circ}$ Annotations sur l'emploi des plantes phanéragomes des Antilles de la flore du Père Duss par M. Heckel (I 896).

$6^{\circ}$ Les plantes médicinales et toxiques de la Guyane française (1897) par M. Heckel.

$7^{\circ}$ Du bois piquant (Zanthoxy-lum Perrottetii D. C. (1 897).

$8^{0}$ Sur la constitution chimique et l'action physiologique du rouge de kola, comparaison avec la caféine (M. Heckel).

$9^{\circ}$ Expériences comparatives concernant l'action du kola et de la caféine sur la fatigue et l'essoufflement déterminés par les grandes marches. (M. Heckel 1890 ).

$10^{\circ}$ Un faux kola nouveau recherches sur les graines de Pentadesma butyracea qui tournissent le beurre de kanya et sur l'emploi de ces semences pour sophistiquer celles du kola (Sterculia acuminata) (Repert. pharmacie i 888).

I I ${ }^{0}$ Sur l'Araucaria brasiliensis son rendement, son acclimatation en Europe et en Algérie. (M. Heckel, Revue sciences naturelles 1892 ).

Sur le Bunya-Bunya (Araucaria Bidwilli) son utilité et son acclimatation en Algérie et dans nos colonies françaises (Revue des Sciences naturelles août 1891). 
I30 Sur Je Copaifera salikounda et sur ses graines à coumarine salikounda des peuples Sousous (Afrique tropicale) au point de vue botanique et chimique, comparaison avec la fève Tonka. (ann. de la fac. des Sciences 1892).

$14^{\circ}$ Rapport sur l'organisation de l'Enseignement Colonial dans les Universités Françaises (18y8 M. Heckel),

$15^{\circ}$ Le Maloukang ou ankalaki de la Côte Occidentale d'Afrique (Polygala butyracea Heck). (Bulletin société géographie. Marseille) 1885.

${ }_{16} 6^{\circ}$ Inée. - Matière médicale et thérapeutique. Dictionnaire encyclopédique des sciences médicales.

I $7^{\circ}$ Etude sur le M'Bentamaré (Cassia occidentalis) de la còte occidentale d'Afrique. Café nègre. Archires de méd. navale 1885.

18" Etude de nouvelles plantes médicinales néo-calédoniennes, Résine de Gardénia. Gomme résine de gardénia, produits des Spermelopis (Chə̀ne-gomme). Heckel et Schlagdenhauffen du (Répert. pharmacie 1893 ).

$19^{\circ}$ Nourelles recherches botaniques chimiques et thérapeutiques sur le Baobab (Adansonia digitata.) Heckel et Schlagdenhauffen (Nouveaux remèdes i 838 ).

$20^{\circ}$ Sur la Courbarii et son fruit. (Heckel et Schlagdenhauffen dans (Le naturaliste 1889 ).

$21^{\circ} \mathrm{L}$ geertnera vaginata et sur ses graines considérées comme un vrai café (Heckel et Schlagdenhauffen. Répert. pharmacie 1890).

$22^{\circ}$ Sur le tulercule aérien du dioscorea hoffa. lleckel et Schlagdenhauffen (bulletin soriété nationale acclimatation 1889).

$23^{\circ}$ Sur deux plantes alimentaires coloniales peu connues. Dios corex bulbifira et tacca involucrata (Rerue sciences naturelles. Heckel et Schelagdenhauffen 1892).

$24^{\circ}$ Sur le férier (gleditschia triacanthos) Heckel et Schlagdenhauffen (Repert. pharmacie. Jans. 1893 ).

$25^{\circ}$ Sur le processus germinatif dans la graine de ximenia américana et sur la nature des écailles rudiciformes propres à cette espèce (Heckel. Rerue générale botanique 1899).

$26^{\circ}$ De l'emploi des feuilles de combretum raimbaulti contre la 
fièvre bilieuse hématurıque des pays chauds (Heckel. Repert, pharmacie. Juin r $89 \mathrm{r}$ ). Kinkelibah.

$27^{\circ}$ Sur les Psathura de l'île de la Réunion et sur le Psathura angustrfolia en tant que plantes aromatiques excitantes (Heckel et Schlagdenhauffen Repert. pharmacie, fév. r9oo).

$28^{\circ} \mathrm{La}$ Sterculia tomentosa et la gomme de M. Beppe qu'il fournit (M. Heckel. Répert. pharmacie, janv. et fév. r 899).

$29^{\circ}$ Sur la graine de Ko-Sam (Brucea sumatrana et sur sa constitution chimique (par Heckel et Schlagdenhauffen du Répert. pharmacie avril rgoo). 


\section{LISTE}

DES

TRAVAUX DE BOTANIQUE ÉGONOMIQUE COLONIALE PUBLIÉS EN CES DERNTÉRES ANNÉES PAR M. .UMELLE.

I" Le Saliaré (Comptes rendus du Congrès de l'Association française pour l'avancement des Sciences. Carthage 1896 ).

2" Etude du Cissus gongylodes du Brésil (Revue générale de botanique, 1897).

30 Le n'djembo, liane à caoulchouc du Fernan-Vai (Comptes rendus de l'Académie des Sciences, juin 1897).

$4^{0}$ Sur la structure et la germination du Landolphia Foreti (Annales de la Faculté des Sciences de Marseille, 1897).

$5^{\circ}$ L'Erouma de Nounelle-Calédonie et son produit résineux (Annales du Musée colonial de Marseille, 5 volume.)

6" L'Okouendi n'gou'a liane à caoutchouc du Fernan-Taz (Comptes rendu de l'Association française. Saint-Etienne 1897 ).

\% Les plantes à caoutchouc de la Casamance (Revue générale des Siciences, 1898 ).

$8^{\circ}$ sur lacclimatation du Castilloa elastica daus les colonies françaises (Revue générale des Sciences. nov. 1 898).

$9^{\circ}$ Les plantes à caoutchouc et à gutta dans les colonies françaises. (r volume de i 84 pages chez Challamel, éditeur.

I $0^{\circ}$ Sur la coagulation des laits à caoutchouc (Revue des cultures coloniales, 1899 ).

$11^{\circ}$ La détermination chimique de sortes commerciales de cacaos (Revue des cultures coloniales, i 899).

$12^{\circ}$ Le guidroa arbre à caoutchouc de Madagascar (Comptes rendus de l'Académie des Sciences. I 899 ).

130 Le Cryptostegia madagascariensis ou lombiro de Madagascar (Revue des cultures coloniales, 1899).

$14^{\circ}$ Le piralahy, liane à caoutchouc de Madagascar (Comptes rendus de l'Académie des Sciences, I 899).

I $5^{\circ}$ Le casayer saculture et son exploitation dans tous les pays de production, I volume de 210 pages chez Challamel, 1900.

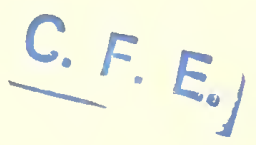


${ }_{16}$ Un Landolphia à caoutchouc de Madagascar (Revue des cultures août 1899).

$17^{\circ}$ Le Marsdenia verrucosa du bokalahy de Madagascar (Revue des cultures coloniales, i 900$)$.

\section{PARAITRONT PROGHAINEMENT :}

Les cultures coloniales, I volume de 5 oo pages, chez Baillière et fils éditeurs.

L'arbre à thé et les végétaux similaires, I volume de 200 pages, environ. chez Challamel. 


\section{COUP-D'EIL MÉTHODIQUE \\ SUR L'ENSEMBLE DES COLLECTIONS DU MUSÉE COLONIAL}

Lespace trop parcimonieusement mesuré dont on disposait pour l'Exposition de l'Institut Colonial de Marseille, n'a pas permis d'y faire figurer tout ce qui aurait pu faire ressortir à sa réelle valeur l'importance de cet établissement dù à l'initiative privée. Aussi devons-nous indiquer sommairement ce qu il contient et ce qui offre le plus grand intérêt, non-seulement à ceux qui sont à la recherche de l'utilisation des ressources coloniales, mais encore à ceux qui par simple curiosité aiment à s’instruire au point de vue ethnographique.

Produits commerciaux. - Les Bois de nos différentes colonies y sont exposés, soit en billes munies de leur écorce, avec une tranche polie et vernie qui permet de voir les veines, soit en rouelies, ou encore en petites plaques faisant bien ressortir par le fini du travail ce qüils valent pour l'ébénisterie, la marqueterie ou la sculpture. On peut voir ainsi la collection des bois de $1^{\circ}$ L'Annam; $2^{\circ}$ de la Nouvelle Calédonie; $3^{\circ}$ du Cambodge ; $4^{\circ}$ cie la Côte d'Ivoire; $5^{\circ}$ de la Guyane Française ; $6^{\circ}$ de l'Inde; $7^{\circ}$ de Madagascar; $8^{\circ}$ de la Réunion. Outre les bois précieux : de rose, d'ébène. de palissandre, d'acajou, de fer, de violette, la collection contient tous les bois employés dans l'industrie du parfumeur pour les huiles essentielles qu'ils cèdent à la distillation : santal, myoporum, bielschmiédia, bois d'Inde. Ceux qui donnent du feu par le simple frottement, d'autres qui rendent l'eau savonneuse, ceux jenfin qui produisent des substances tannantes comme le Spermolepis tannifera, et celu qui sert pour faire des cercueils à Madagascar dont la propriété est de conserver les cadavres à l'abri de la putréfaction.

Graines oléagineuses. - La collection des graines grasses est sans contredit la plus complète de toutes celles qui existent. La grande industrie de Marseille des huiles et des savons étant à la recherche de toutes les zraines qui alimentent son travail, le laboratoire de l'Institut Colonial de Marseille s'est fait une spécialité de 
l'étude des graines grasses, aussi dès qu une graine inconnue arrive sur le marché est-elle envoyée dans ce laboratoire pour en faire déterminer l'espèce botanique et la valeur commerciale et industrielle. On peut aisément s'en convaincre en examinant la grande variété des corps gras nouveaux exposés avec leurs dérivés : acides gras de distillation, de saponification, stéarines de distillation, stéarines de saponification, glycérines et tourteaux provenant de graines inconnues et inutilisées jusqüici.

Lá nomenclature en serait trop longue elle demanderait des volumes. Qu'il nous suffise de citer seulement le krébao, le méné dont on peut voir des bougies, l'owala qui arrive par chargements depuis les traraux de $\mathrm{M}$. Heckel qui l'ont fait connaitre au commerce et à l'industrie, avec saron et bougies, le maloukang arec son beurre. l'ochoco, le kombo, le d'jareéou n'javé, etc etc....

Matières tinctoriales. - A côté des nourelles matières comme le doundaké qui teint en jaune et le zanthoxylum perrotettii, on peut voir toutes les espèces de cochenilles, les dividivi. les orseilles, les campêches, les fustet, le curcuma, l'indigo, le rocou, les cachous etc etc.

Parfumerie - Les essences d'ylang-ylang, de géranium, de santal, de myoporum, de bielschmiedia anisiodora, de myrtus, pimenta, de faux poirrier, extrait de vanille, de vétiver, de Basilic etc etc....

Epices. - Poivre long, poirre noir, poirre blanc, piment jamaïque, piment enragé, noix de ravensara, noix muscades, girofles, gingembre gris, gingembre blanc, curcuma, macis, cardamomes, cannelles, bétel, piment couronné, etc.

Matières alimentaires. - Végétaline extraite du coprah. café nègre de M'. Bentamaré ce dernier faisant l'objet d'une spécialité de café chicorée exploité par une société depuis les études faites sur cette graine par le Dr Heckel. Cacaos de toutes provenances, cafés : de Bourbon, de la Martinique, de Calédonie, du Congo, de Taïti. Toutes les sortes de mil du Soudan et du Sénégal. Le manioc, l'arrow-root, la farine de moussache, la farine desséchee de la pulpe de Baobab, les ambrevades etč etc. 
Textiles. - Les fibres textiles sont en très grand nombre: Les soies du Tonkin, de l'Annam, les fibres de ramie, de Madagascar, accompagnées de fort jolis échantillons de tissus. Le bourao, chanvre de Manille, fibres de bananier, de cocotier.

Les fibres de raphia de Madagascar avec des rabanes tissées dans le pays. Fibres de Baobab, d'alò̀s, de Jute de Magnagna. Toutes les espèces de coton de différentes provenances.

Produits scientifiques: La Nicouline et ses sels retirée du Robinia Nicou. La Kolanine extraite du kola vrai. La Colombine du Bakis plus abondante que dans le Colombo. La Sangoline et la Pélosine retirée du sangol, cocculus Iraeeba etc, etc.

Collections ethnographiques. - Religion : Tabous de Nouvelle Calédonie. Fétiches calédoniens et des Pahouins. - Gris-gris du Soudan, du Dahomey. - Grelots de sorciers. - Livre de prières. Touareg (en bois). - Amulettes Laotiennes : dents d'ours, cornes de cerf, dents de tigres. - Chapelets Boudhiques. - Baguettes d'encens boudhistes; images annamites pour chasser les mauvais esprits. génie des navigateurs annamites. - Diplômes mortuaires du Tonkin. - « Le bay Mahal » fac-simile du tombeau de Shah-Jehan en albâtre. - Bois d'agallocha pour brûler dans les pagodes. etc etc.

Guerre : Casse-tète canaques et Galibis, riche collection. - Sagaies calédoniennes et africaines. - Sarbacanes. - Arcs des Mois avec flèches. - (Laos) - arc et flèches de Madagascar. - Fusils et obus Malgaches. - Pistolet Touareg. - Sabres du Tonkin, du Laos, de l'Annam. - Fusils de pirates. - Trompettes d'alarme de pirates. Bàtons de commandement. - Sabres d'exécution. - Masques de guerre. - Carquois Galibis avec flèches empoisonnées au curare. Flèches empoisonnées au Tanghin. - Boucliers Pahouins. Boucliers de pirates chinois. - Couteaux de jet du Congo. - Sabres Pahouins, hache Malgache. - Coupe-coupe divers etc. etc. Cornes à poudre en ivoire d'Obock. - Matelas du Tonkin pour préserver des balles.

Musique: Balafons du Soudan. - Viati tama Tam-tam du Soudan). - Tam-tam des Ouassoulous (Soudan). - Harpes des Bavilis (Congo). - Tam-tam du Congo. - Gong Laotien. - (Lou- 
sen flûtes Laotiennes. - Flûtes du Tonkin. - Tam-tam MuongVahlias Malgaches. - Caïambe et castagnettes Malgaches. - Bobr instrument de musique Malgache.

Ustensiles de travail : Pirogues et pagayes. - Fuseau à filer le coton du Dahomey. - Pioches pour cultiver la terre du Soudan appelées: Diélé, servant avec le Guiéla à défricher. - Daba servant à arracher les arachides. - Darambo - Certaines de ces pioches sont en dents d'éléphants, d'autres en pierre de serpentine. - Le Soumbé est une large pioche pour préparer le terrain au Soudan. Haches en serpentine des Pahouins et des Canaques. Instruments agricoles de Cochinchine pour la culture du riz. - Roue à niveler les rizières, pièce à repiquer le riz et couteau à main pour le couper lors de la récolte.

Des charettes annamites pour les transports agricoles. - Des navettes à tisser le coton du Tonkin. - Harpons pour la pèche avec pointes empoisonnées. - Hameçons canaques en nacre. Pompe à incendie Tonkinoise. - Malles et hottes Muong en vannerie pour le transport des effets et des marchandises. - Pièces de toile servant aux femmes Méo (Tonkin) à porter leurs enfants sur le dos, travail remarquable d'application de divers tissus en couleur formant des dessins symétriques d'un très joli effet.

Habitation : modèles de paillottes des Galibis. -- Cases de Madagascar, etc., etc.

Ustensiles de ménage: Baguettes à manger le riz annamites. Couteaux et boìtes à bétel. - Calebasses du Soudan peintes. sculptées des Galibis (mère d'Apatou), - Calebasses volumineuse creusée dans un tronc de baobab. - Porcelaine du Laos. - Gargoulettes malgaches artistiques. (Céramique artistique des Galibis, belle collection). - Céramique malgache, annamite, tonkinoise et indienne. - Remarquable collection de fruits des tropiques en terre cuite peinte. - Nusettes muong. - Marmites canaques. Mortiers à piler le riz et les couscouss. - Cuillères à riz.

Vêtements : Pagnes du Dahomey. - Tapas des femmes canaques. - (Costumes du Haut-Tonkin. Ta-pan-yan). - Riche collection de costumes et tissus laotiens : Sampots de soie, velours. - Coussins 
annamites. - Pantalons, vestes, turbans, robes, jupes, guètres et coiffures des Annamites. - Tablier brodé de Lao-Kay. - Riches robes de Pagodes brodées d'or. - Etoffe. présent de noce en écorce de banian.

Chaussures: Très riche collection de toutes les chaussures de l'Extrême-Orient, du Soudan, des Touaregs, de Madagascar etc., etc.

Coiffures: bonnets des Toucouleurs, des Bambaras, des Malinkès, bonnets de pagodes Tonkin. - Coiffures de mandarins, de guerriers pahouins. - Grande variété de chapeaux de paille pour travailler dans les rizières, pour les fêtes, etc., etc.

Coiffures du Haut-Tonkin (Ta-pan-yao) en écharpes. - Toques malgaches tissées en paille de riz.

Pipes à opium. - Pipes à eau du Tonkin. - Pipes en terre et en bambou annamites.

Boîtes à bijoux annamites. - Boîtes à bétel en cuivre et en bois. - Encriers. - Machine à compter. - (Poids annamites. - Balances pour l'or).

Vannerie du Tonkin, du Laos, du Sénégal, de Madagascar, en rotin, en bambou et en paille de riz.

Peignes et miroirsannamites. - Eventails. - Ciseaux.

Serviettes en raphia du Congo (Brazzaville).

Dentelles en soie de Madagascar.

Les herbiers des colonies comprenant:

La Réunion

La Nouvelle-Calédonıe

Côte occidentale d'A frique: Gabon
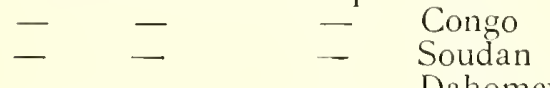

Madagascar(collection Prince)

Haut-Laos (collection Dupuy)

Tonkin (collection du Dr Simon)

Taîti (collection du Dr Vincent)

Cambodge (collection Collard)

Antilles (herbier du R. P. Duss)

Guyane (collection Bessières)

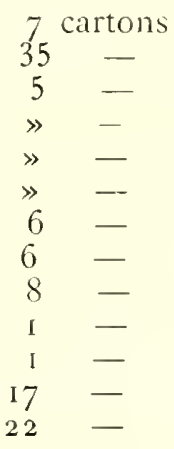


Les collections minéralogiques notamment celles de l'Algérie et de la Nouvelle-Calédonie ont une véritable importance et remplissent deux grandes vitrines horizontales. Celle de Madagascar est encore insuffisante, mais il faut remarquer un bloc de cristal de roche du poids de 55 kilos donné par M. Le-Fol, commissaire général des colonies actuellement au Tonkin. 


\section{TABLE DES MATIERES}

Pages

Historicue. . . . . . . . . . . . . . . . . . . 1

Musce Colonial . . . . . . . . . . . . . . . . . . . . 7

Collection des produits d'Afrique . . . . . . . . . . . . . 18

Collection des prortuits d'Amrique . . . . . . . . . . . . . 17

Collection des produits le l'Océanie. . . . . . . . . . . . 19

Collection des produits d'Asie . . . . . . . . . . . . . 20

Glassement des collections. . . . . . . . . . . . . . . 21

Institut Colonial. . . . . . . . . . . . . . . . . . 23

Nouvel Institut Colonial projetí jar la Municipalité de Marseille. $\quad 31$

Résumé des travaux botaniques les plus récents, faits au laboratoine de l'Institut Colonial . . . . . . . . . . . . . . . . . . 39

Les caoutchoues des colonies frangaises et notamment ceux fui sont exposés par le Musée Colonial . . . . . . . . . . . . 11

Gomme et Gommes résines des colonies françaises par M. J. de Cordemoy . . . . . . . . . . . . . . . . . . 51

Graines grasses nourelles ou jeu connues, notamment celles qui figurent à l'exposition du Trocadéro et provenont des colonies francaises par M. Ed. Herkel. . . . . . . . . . . . 55

Le tahac dans les colonies, par M. Laurent. . . . . . . . . . . 59

Résumé des travaux zoologiques de l'Institut Colonial. . . 69

Le grillide qui ravage les plantations de cafe. . . . . . . . . . . T1

Etude du fourreau d'une espece de psychidé des environs de Tombouctou. . . . . . . . . . . . . . . . . 75

Les acridiens, études anatomiques et biologiques, déveloplement. migrations, dégâts considèrables qu'ils causent à l'agriculture. moyens employés pour les combattre . . . . . . . . . . . 96 
Nomenclature des produits exposés par l'Institut Colonial avec les indications du groupe et de la classe auxquels ils appartiennent. Liste des donateurs du Musée Colonial, donateurs en espèces. . . 89 Listes des donateurs du Musce Colonial, dons en nature . . . . . 93 Nomenclature des travaux publiés par M. Heckel et Schlagdenhauffen lans les dernicres années en dehors des Annales. . . . . . . .

T'ravaux de botanique économique coloniale publiés en ces dernières années par M. Jumelle. . . . . . . . . . . . . . . . . 101 Coup l'wil métholique sur l'ensemble des collections du Musée Colonial.

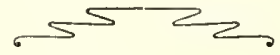






SPECIML $\begin{aligned} 93-13 \\ 7475\end{aligned}$ 


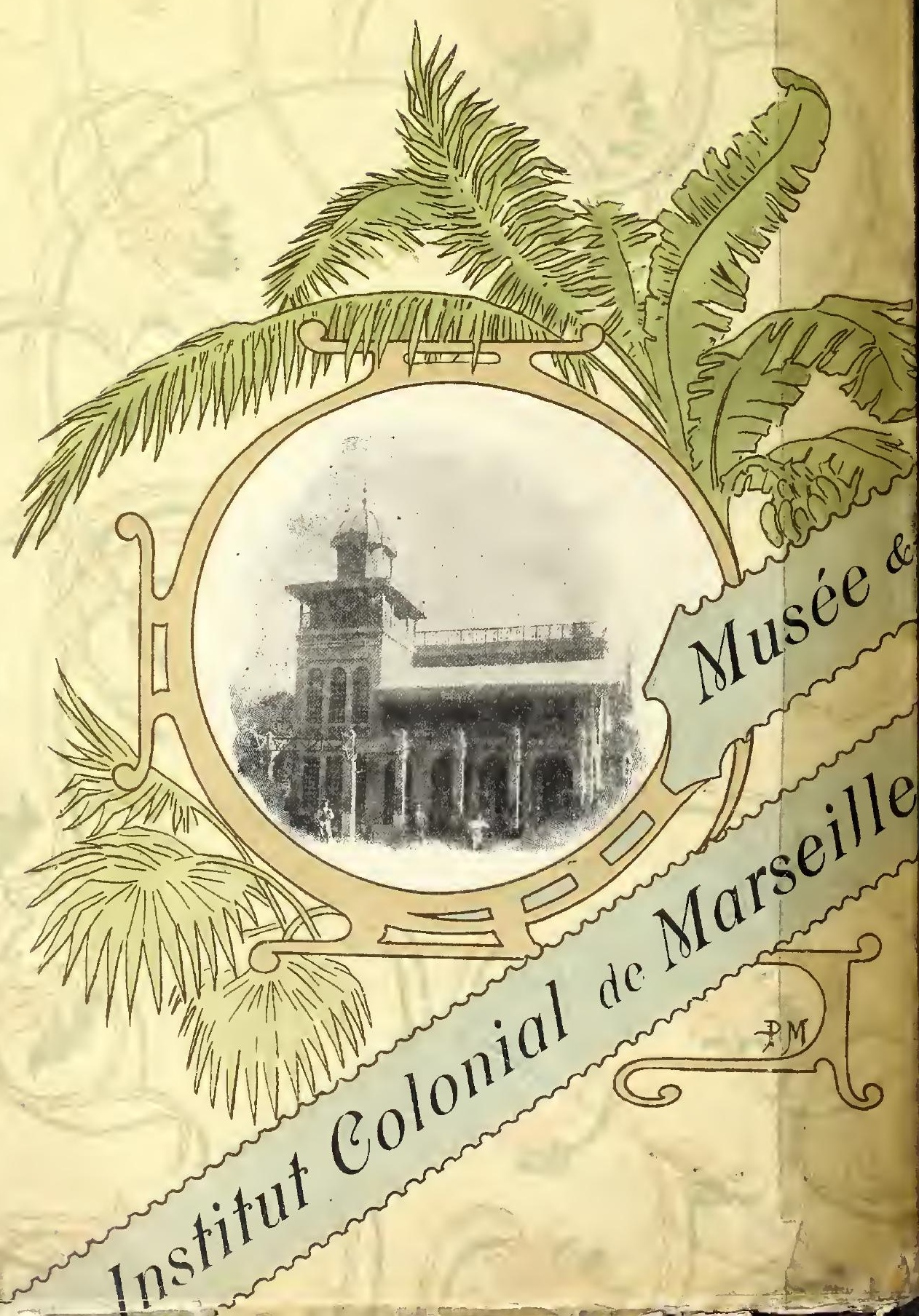

